\title{
The Abel map for surface singularities I: generalities and examples
}

\author{
János Nagy ${ }^{1}$. András Némethi ${ }^{2,3,4}$ \\ Received: 11 September 2018 / Revised: 4 June 2019 / Published online: 30 July 2019 \\ (c) The Author(s) 2019
}

\begin{abstract}
Let $(X, o)$ be a complex normal surface singularity. We fix one of its good resolutions $\widetilde{X} \rightarrow X$, an effective cycle $Z$ supported on the reduced exceptional curve, and any possible (first Chern) class $l^{\prime} \in H^{2}(\widetilde{X}, \mathbb{Z})$. With these data we define the variety $\mathrm{ECa}^{l^{\prime}}(Z)$ of those effective Cartier divisors $D$ supported on $Z$, which determine a line bundles $\mathcal{O}_{Z}(D)$ with first Chern class $l^{\prime}$. Furthermore, we consider the affine space $\mathrm{Pic}^{l^{\prime}}(Z) \subset H^{1}\left(\mathcal{O}_{Z}^{*}\right)$ of isomorphism classes of holomorphic line bundles with Chern class $l^{\prime}$ and the Abel map $c^{l^{\prime}}(Z): \mathrm{ECa}^{l^{\prime}}(Z) \rightarrow \mathrm{Pic}^{l^{\prime}}(Z)$. The present manuscript develops the major properties of this map, and links them with the determination of the cohomology groups $H^{1}(Z, \mathcal{L})$, where we might vary the analytic structure $(X, o)$ (supported on a fixed topological type/resolution graph) and we also vary the possible line bundles $\mathcal{L} \in \mathrm{Pic}^{l^{\prime}}(Z)$. The case of generic line bundles of $\operatorname{Pic}^{l^{\prime}}(Z)$ and generic line bundles of the image of the Abel map will have priority roles. Rewriting the Abel map via Laufer duality based on integration of forms on divisors, we can make explicit the Abel map and its tangent map. The case of superisolated and weighted homogeneous singularities are exemplified with several details. The theory has similar goals (but rather different techniques) as the theory of Abel map or Brill-Noether theory of reduced smooth projective curves.
\end{abstract}

Keywords Normal surface singularity · Resolution graph · Rational homology sphere · Natural line bundle $\cdot$ Poincaré series - Abel map · Effective Cartier divisor . Picard group · Brill-Noether theory $\cdot$ Laufer duality $\cdot$ Surgery formulae $\cdot$ Splice quotient singularity $\cdot$ Superisolated singularity $\cdot$ Weighted homogeneous singularity

Mathematics Subject Classification Primary 32S05 - 32S25 - 32S50 - 57M27; Secondary $14 \mathrm{Bxx} \cdot 14 \mathrm{~J} 80$

Communicated by Jean-Yves Welschinger.

Extended author information available on the last page of the article 


\section{Introduction}

In this introduction we plan to provide the major ideas and some of the major results without technical details. The presentation will automatically provide the structure of the article as well.

The study of the Abel map of projective irreducible smooth curves was a crucial tool in the classical algebraic geometry and it remained so in the modern theory as well. Though in this work we will use very little from this theory, in this introduction (and some places later) we will discuss some comparisons between the curve case and the theory of the present article established for normal surface singularities, mostly to emphasize the major conceptual differences and additional difficulties in the later case. (For the Abel map of curves one can consult [1] and the references therein.)

The present manuscript is the first one in a series of articles planned (and partly already written) by the authors. It contains the foundation, the presentation of the basic constructions and of the basic properties. They are also supported by several examples. The forthcoming manuscripts of the series treat the theory applied for several important families of singularities, e.g. for singularities with generic analytic type, or elliptic or splice quotient singularities. E.g., in the second article [32], based on the results of the present one, we treat properties of the generic analytic structure supported by a fixed resolution graph (topological type). More precisely, we are able to determine topologically several discrete analytic invariants of such singularities like multivariable Hilbert series associated with the divisorial filtration, or cohomology of cycles and line bundles supported on the fixed resolution (in particular, the geometric genus as well).

We wish to emphasize from the start that we are not generalizing the Abel construction from the curve case to the - smooth or singular-(quasi)projective surfaces: our goal is to develop its analogue valid in the context of a resolution of a complex normal surface singularity germ. This means that if $(X, o)$ is such a singularity with a fixed good resolution $\widetilde{X} \rightarrow X$, then for any effective cycle $Z$ supported on the reduced exceptional curve $E$ and for any (possible) Chern class $l^{\prime} \in H^{2}(\tilde{X}, \mathbb{Z})$ we construct the space $\mathrm{ECa}^{l^{\prime}}(Z)$ of effective Cartier divisors $D$ supported on $Z$, whose associated line bundles $\mathcal{O}_{Z}(D)$ have first Chern class $l^{\prime}$. Furthermore, we consider the space $\mathrm{Pic}^{l^{\prime}}(Z) \subset H^{1}\left(\mathcal{O}_{Z}^{*}\right)$ of isomorphism classes of holomorphic line bundles with Chern class $l^{\prime}$ and the Abel map $c^{l^{\prime}}(Z): \mathrm{ECa}^{l^{\prime}}(Z) \rightarrow \operatorname{Pic}^{l^{\prime}}(Z), D \mapsto \mathcal{O}_{Z}(D)$. In this way, our Abel map is associated with non-reduced projective curves supported by the exceptional set of a good resolution of a normal surface singularity. In particular, the combinatorial background is the combinatorics of the dual resolution graph $\Gamma$ (or the intersection from (, ) of the irreducible exceptional curves), that is, equivalently, the 3-dimensional link of the singularity. In fact, in order to run properly the theory (e.g. to be able to define the 'natural' line bundles, cf. 2.3), we will even assume that the link of the singularity is a rational homology sphere. This happens exactly when the resolution graph $\Gamma$ represents a tree of rational curves. In this way, in all the discussions regarding the analytic types and properties we move the difficulties from the moduli space of each irreducible exceptional curve $E_{v}$ (which is trivial in this case) to the analytic properties of their infinitesimal tubular neighbourhoods and their gluings (analytic plumbing). 
Therefore, the Abel map $c^{l^{\prime}}$ behaves rather differently than the (projective) Abel map of reduced smooth curves, it shares more the properties of non-proper affine maps rather than the projective ones. This will also be clear from the next preliminary presentation of its source and target.

In fact, the space $\mathrm{ECa}^{l^{\prime}}(Z)$ is already constructed in the literature. Note that by a theorem of Artin [4, 3.8], there exists an affine algebraic variety $Y$ and a point $y \in Y$ such that $(Y, y)$ and $(X, o)$ have isomorphic formal completions. Then, according to Hironaka [18], $(Y, y)$ and $(X, o)$ are analytically isomorphic. In particular, we can regard $Z$ as a projective algebraic scheme, in which situation $\mathrm{ECa}^{l^{\prime}}(Z)$ was constructed by Grothendieck [16], see also the article of Kleiman [20] and the book of Mumford for curves on algebraic surfaces [31]. In particular, $\mathrm{ECa}^{l^{\prime}}(Z)$ is a quasiprojective variety. Though the existence of the space $\mathrm{ECa}^{l^{\prime}}(Z)$ in this way is established, we will provide several key properties valid in our particular situation, including the local charts. E.g., we will characterize topologically when the space $\mathrm{ECa}^{l^{\prime}}(Z)$ is nonempty $\left(\mathrm{ECa}^{l^{\prime}}(Z) \neq\right.$ $\emptyset$ if and only if $-l^{\prime}$ belongs to the Lipman cone, cf. (3.1.5)). In Theorem 3.1.10 we prove the following.

Theorem If $-l^{\prime}$ belongs to the Lipman cone then the following facts hold.

(1) $\mathrm{ECa}^{l^{\prime}}(Z)$ is a smooth complex irreducible variety of dimension $\left(l^{\prime}, Z\right)$.

(2) The natural restriction $r: \mathrm{ECa}^{l^{\prime}}(Z) \rightarrow \mathrm{ECa}^{l^{\prime}}(E)$ is a locally trivial fiber bundle with fiber isomorphic to an affine space. Moreover, the homotopy type of $\mathrm{ECa}^{l^{\prime}}(Z)$ is independent of the choice of $Z$ and it depends only on the topology of $(X, o)$.

The fibers of $r: \mathrm{ECa}^{l^{\prime}}(Z) \rightarrow \mathrm{ECa}^{l^{\prime}}(E)$ can be considered as certain jet spaces in the local infinitesimal neighbourhoods of the local equations of the effective Cartier divisors. Since they are affine spaces, $\mathrm{ECa}^{l^{\prime}}(Z)$ is not projective. In fact, even $\mathrm{ECa}^{l^{\prime}}(E)$ usually becomes non-projective too; e.g. for $l^{\prime}=-E_{v}^{*}$ (the dual of $E_{v}$, representing 'cuts' which intersects $E_{v}$ but not the other curves) we get $\mathrm{ECa}^{l^{\prime}}(E)=E_{v} \backslash \cup_{u \neq v} E_{u}$.

Note also that the base space $\mathrm{Pic}^{l^{\prime}}(Z)$ is also noncompact, it is an affine space of dimension $h^{1}\left(\mathcal{O}_{Z}\right)$. (Here the assumption that the link is a rational homology sphere plays a role; otherwise $\operatorname{Pic}^{l^{\prime}}(Z) \simeq H^{1}\left(\mathcal{O}_{Z}\right) / H^{1}(\widetilde{X}, \mathbb{Z})$ would have a complex torus component as well). This affine structure will be exploited deeply in the body of the paper. Finally we also mention that the Abel map itself is algebraic, and in fact its (rather non-trivial) expression in local charts can be done explicitly via Laufer duality (integrating forms along divisors in $\widetilde{X}$ ), for details see Sect. 7.

Since the Abel map is not proper, its image usually is not closed, and it can be a rather complicated constructible set (it can be singular as well, cf. Example 12.9.1). In this note we give several examples and also we characterize the dimension of this image. It is not topological, usually it depends in a subtle way on the analytic structure of the singularity. In order to show the presence of possible anomalies we list several examples based on the theory of elliptic and splice quotient singularities (certain familiarity with them might help essentially the reading).

E.g., in Example 3.4.3 we provide an example when the Abel map $c$ has the following description. Take the blow up $b: B \mathbb{C}^{2} \rightarrow \mathbb{C}^{2}$ of $\mathbb{C}^{2}$ at the origin. Let $L_{x}$ and $L_{y}$ be the strict transforms of $\{x=0\}$ and $\{y=0\}$. Then in that case $\mathrm{ECa}^{l^{\prime}}(Z)$ can 
be identified with $B \mathbb{C}^{2} \backslash\left(L_{x} \cup L_{y}\right)$ and $c$ with the restriction of $b$ to this space. In this case $\operatorname{im}(c)$ is homeomorphic to $\left(\mathbb{C}^{*}\right)^{2} \cup\{(0,0)\}$.

We also show that all the fibers of $c^{l^{\prime}}$ are smooth (irreducible, quasiprojective), however, their dimensions might jump. The dimension of $c^{-1}(\mathcal{L})\left(\mathcal{L} \in \operatorname{Pic}^{l^{\prime}}(Z)\right)$ is $h^{0}(Z, \mathcal{L})-h^{0}\left(\mathcal{O}_{Z}\right)=\left(l^{\prime}, Z\right)+h^{1}(Z, \mathcal{L})-h^{1}\left(\mathcal{O}_{Z}\right)$. Any fiber appears as quotient by the algebraic free proper action of $H^{1}\left(\mathcal{O}_{Z}^{*}\right)$, which, as algebraic variety, has dimension $h^{1}\left(\mathcal{O}_{Z}\right)$. (This also shows a major difference with the curve cases, where the space of effective divisors associated with a bundle has the form $H^{0}(\mathcal{L}) \backslash\{0\}$, and the action is the projectivization action of $\mathbb{C}^{*}$. In particular, the fibers are projective spaces.) The above relation makes the connection with another major problem/task of the theory, namely determination of possible values of $h^{1}(Z, \mathcal{L})$.

This ' $h$ ' -problem can be formulated even independently of the Abel map, and in fact, it was our most important motivation. Let us fix a topological type (say, the resolution graph $\Gamma$ ), and we consider an arbitrary analytic type of singularity and its resolution supported by $\Gamma$. Then for fixed Chern class $l^{\prime}$ and cycle $Z$ we can also consider all the possible line bundles $\mathcal{L} \in \mathrm{Pic}^{l^{\prime}}(Z)$. The challenge is to determine all the possible values of $h^{1}(Z, \mathcal{L})$, and understand/organize them is a conceptual way. This can be split in two major steps: in the first case one varies all the analytic structures (both of $(X, o)$ and of the line bundles), in the second case one fixes an analytic structure $(X, o)$ (and one of its resolutions $\widetilde{X}$ ) and one moves $\mathcal{L} \in \operatorname{Pic}^{l^{\prime}}(Z)$. E.g., in this second case, one can ask for the stratification $\cup_{k} W_{l^{\prime}, k}$ of $\operatorname{Pic}^{l^{\prime}}(Z) \simeq H^{1}\left(\mathcal{O}_{Z}\right)$ by $W_{l^{\prime}, k}=\left\{\mathcal{L}: h^{1}(\mathcal{L})=k\right\}$. (These are the analogues of the Brill-Noether strata. For the Brill-Noether theory see $[1,13]$.) Or, one can search for the possible values $k$ when $W_{l^{\prime}, k} \neq \emptyset$. In the body of the article we will provided several bounds and partial results (with sharp lower bounds provided by generic structures). Though the older previous results in normal surface singularities focus mostly on particular analytic structures (rational, elliptic, weighted homogeneous, splice quotient, etc), and to special line bundles (e.g. of type $\mathcal{O}_{Z}(l)$ ), in the present note we aim to create a theory which helps to attack the general case, e.g. to treat the case of generic analytic structure or the generic line bundles as well.

Part of the results are reduced to the case of Abel maps which are dominant. This case is completely characterized in Theorem 4.1.1:

Theorem Fix $-l^{\prime}$ from the Lipman cone, $Z \geq E$, and consider $c^{l^{\prime}}: \mathrm{ECa}^{l^{\prime}}(Z) \rightarrow$ $\operatorname{Pic}^{l^{\prime}}(Z)$.

(1) $c^{l^{\prime}}$ is dominant if and only if $\chi\left(-l^{\prime}\right)<\chi\left(-l^{\prime}+l\right)$ for all $0<l \leq Z, l \in L$. In particular, the fact that $c^{l^{\prime}}$ is dominant is independent of the analytic structure supported by $\Gamma$ and it can be characterized topologically (and explicitly).

(2) If $c^{l^{\prime}}$ is dominant then $h^{1}(Z, \mathcal{L})=0$ for generic $\mathcal{L} \in \operatorname{Pic}^{l^{\prime}}(Z)$.

For fixed and large $Z$ (in which case $\operatorname{Pic}^{l^{\prime}}(Z)=\operatorname{Pic}^{l^{\prime}}(\tilde{X})$ ) we introduce $\mathcal{S}_{\text {dom }}^{\prime}$ as the set of those Chern classes $l^{\prime}$ for which $c^{-l^{\prime}}$ is dominant, and we list several properties of it. It is a semigroup of the topological Lipman semigroup/cone $\mathcal{S}^{\prime}$, and it has several properties of the analytic semigroups. The study of dominant maps emphasizes again the importance of the study of generic line bundles. In Sect. 5 we will list several cohomological properties for the generic line bundle $\mathcal{L}_{\text {gen }}$ of Pic ${ }^{l^{\prime}}$ (e.g. we determine 
its $h^{1}$ topologically, and we show that this value is a sharp lower bound for any $h^{1}(\mathcal{L})$ ). Similarly, the generic line bundle of the image of the Abel map $c^{l^{\prime}}$ is also studied (its $h^{1}$ is the codimension of $\operatorname{im}\left(c^{l^{\prime}}\right)$ and it is also the sharp lower bound for any $h^{1}(\mathcal{L})$ with $\left.\mathcal{L} \in \operatorname{im}\left(c^{l^{\prime}}\right)\right)$. Upper bounds for $h^{1}(Z, \mathcal{L})$ are also established. E.g. Theorem 5.3.1 and Proposition 5.6.1 imply:

Theorem Fix $Z>0$.

(I) Fix an arbitrary $l^{\prime} \in L^{\prime}$. Then for any $\mathcal{L} \in \mathrm{Pic}^{l^{\prime}}(Z)$ one has

$$
h^{1}(Z, \mathcal{L}) \geq \chi\left(-l^{\prime}\right)-\min _{0 \leq l \leq Z, l \in L} \chi\left(-l^{\prime}+l\right) .
$$

Furthermore, if $\mathcal{L}$ is generic in $\mathrm{Pic}^{l^{\prime}}(Z)$ then the inequality transforms into an equality.

In particular, $h^{*}(Z, \mathcal{L})$ is topological and explicitly computable from $L$, whenever $\mathcal{L}$ is generic.

(II) For any $\mathcal{L} \in \operatorname{im}\left(c^{l^{\prime}}\right) \subset \mathrm{Pic}^{l^{\prime}}(Z)$ one has

$$
h^{1}(Z, \mathcal{L}) \geq h^{1}\left(\mathcal{O}_{Z}\right)-\operatorname{dim}\left(\operatorname{im}\left(c^{l^{\prime}}\right)\right)=\operatorname{codim}\left(\operatorname{im}\left(c^{l^{\prime}}\right)\right)
$$

Furthermore, equality holds whenever $\mathcal{L}$ is generic in the image of $c$.

The Abel map is compatible with additive structure of the divisors and multiplicative structure of the line bundles. The point is that if we consider a sufficiently high multiple of a Chern class (that is, we replace $l^{\prime}$ with $n l^{\prime}$ where $n \gg 0$ ), then the image of $c^{n l^{\prime}}$ becomes an affine subspace for each $n$, and the associated vector subspaces (indexed by $n$ ) stabilize, and this stabilized vector subspace depends only on the 'dual-basesupport' of $l^{\prime}$ (see Theorem 6.1.9). This collection of stabilized linear subspaces (as a linear subspace arrangement) and their dimensions become the source of important new analytic invariants, see Sect. 6. E.g., the dimensions serve as correction terms in our new analytic surgery formulae (see e.g. Theorem 6.1.9). If the analytic structure of $(X, 0)$ is 'nice' (e.g. splice quotient), then these correction invariants can be connected with known analytic invariants computable from the Poincare series of the divisorial filtrations, and in such cases the 'classical' surgery formulae can be recovered or improved (see Sect. 9). It is worth to emphasize that the classical surgery formulae (see e.g. [49], or [6]) are valid for the special 'natural' line bundles and under special analytic conditions, and it was not clear at all if any extension to the general case might exists and/or how to define the correction terms in such general situations. In the present note this is solved via the above stabilized dimensions of the images of Abel maps (without any required restriction). Furthermore, under the special analytic conditions of the old surgery formulae, they are identified with the classical correction terms.

Starting from Sect. 7 we develop the 'duality picture' between divisors and differential forms. This not only describes the Abel map and its tangent map, but it gives a computational tool in concrete examples as well. The invariants of stable case in 
language of differential forms are described in Sect. 8. The general non-stable case is analyzed in Sect. 10.

When a concrete basis of $H^{0}\left(\widetilde{X} \backslash E, \Omega_{\widetilde{X}}^{2}\right) / H^{0}\left(\widetilde{X}, \Omega_{\widetilde{X}}^{2}\right)$ (dual to $H^{1}\left(\mathcal{O}_{\widetilde{X}}\right)$ ) can be explicitly determined, the Abel map also becomes more transparent, and several of the above listed problems have precise (sometimes even combinatorial) solutions. This is exemplified in the case of superisolated (Sect. 11) and weighted homogeneous (Sect. 12) singularities. Some additional properties in the Gorenstein situation are also listed.

In the sequel \#A denotes the cardinality of the finite set $A$.

\section{Preliminaries}

In this section we review some basic facts about topological and analytical invariants of surface singularities, and we introduce the needed notations as well.

\subsection{The resolution}

Let $(X, o)$ be the germ of a complex analytic normal surface singularity, and let us fix a good resolution $\phi: \widetilde{X} \rightarrow X$ of $(X, o)$. We denote the exceptional curve $\phi^{-1}(o)$ by $E$, and let $\cup_{v \in \mathcal{V}} E_{v}$ be its irreducible components. Set also $E_{I}:=\sum_{v \in I} E_{v}$ for any subset $I \subset \mathcal{V}$. The support of a cycle $l=\sum n_{v} E_{v}$ is defined as $|l|=\cup_{n_{v} \neq 0} E_{v}$. For more details see [22,24,34,35,37].

\subsection{Topological invariants}

Let $\Gamma$ be the dual resolution graph associated with $\phi$; it is a connected graph. Then $M:=\partial \widetilde{X}$ can be identified with the link of $(X, o)$, it is also the oriented plumbed 3-manifold associated with $\Gamma$. It is known that $(X, o)$ locally is homeomorphic with the real cone over $M$, and $M$ contains the same information as $\Gamma$. We will assume that $M$ is a rational homology sphere, or, equivalently, $\Gamma$ is a tree and all genus decorations of $\Gamma$ are zero. We use the same notation $\mathcal{V}$ for the set of vertices, and $\delta_{v}$ for the valency of a vertex $v$.

The lattice $L:=H_{2}(\widetilde{X}, \mathbb{Z})$ is endowed with the natural intersection form (, ), which is negative definite. $L$ is freely generated by the classes of 2-spheres $\left\{E_{v}\right\}_{v \in \mathcal{V}}$. The dual lattice $L^{\prime}:=H^{2}(\widetilde{X}, \mathbb{Z})$ is generated by the (anti)dual classes $\left\{E_{v}^{*}\right\}_{v \in \mathcal{V}}$ defined by $\left(E_{v}^{*}, E_{w}\right)=-\delta_{v w}$ (where $\delta_{v w}$ stays for the Kronecker symbol). The intersection form embeds $L$ into $L^{\prime}$. Then $H_{1}(M, \mathbb{Z}) \simeq L^{\prime} / L$, and it is abridged by $H$. Usually one identifies $L^{\prime}$ with those rational cycles $l^{\prime} \in L \otimes \mathbb{Q}$ for which $\left(l^{\prime}, L\right) \in \mathbb{Z}$, or, $L^{\prime}=\operatorname{Hom}_{\mathbb{Z}}(L, \mathbb{Z})$.

There is a natural (partial) ordering of $L^{\prime}$ and $L$ : we write $l_{1}^{\prime} \geq l_{2}^{\prime}$ if $l_{1}^{\prime}-l_{2}^{\prime}=$ $\sum_{v} r_{v} E_{v}$ with all $r_{v} \geq 0$. We set $L_{\geq 0}=\{l \in L: l \geq 0\}$ and $L_{>0}=L_{\geq 0} \backslash\{0\}$.

Each class $h \in H=L^{\prime} / L$ has a unique representative $r_{h}=\sum_{v} r_{v} E_{v} \in L^{\prime}$ in the semi-open cube (i.e. each $r_{v} \in \mathbb{Q} \cap[0,1)$ ), such that its class $\left[r_{h}\right]$ is $h$. 
All the $E_{v}$-coordinates of any $E_{u}^{*}$ are strictly positive. We define the Lipman cone as $\mathcal{S}^{\prime}:=\left\{l^{\prime} \in L^{\prime}:\left(l^{\prime}, E_{v}\right) \leq 0\right.$ for all $\left.v\right\}$. As a monoid it is generated over $\mathbb{Z}_{\geq 0}$ by $\left\{E_{v}^{*}\right\}_{v}$.

The multivariable topological Poincaré series is the Taylor expansion $Z(\mathbf{t})=$ $\sum_{l^{\prime}} z\left(l^{\prime}\right) \mathbf{t}^{l^{\prime}}$ at the origin of the rational function

$$
\mathcal{Z}(\mathbf{t})=\prod_{v \in \mathcal{V}}\left(1-\mathbf{t}^{E_{v}^{*}}\right)^{\delta_{v}-2},
$$

where $\mathbf{t}^{l^{\prime}}:=\prod_{v \in \mathcal{V}} t_{v}^{l_{v}^{\prime}}$ for any $l^{\prime}=\sum_{v \in \mathcal{V}} l_{v}^{\prime} E_{v} \in L^{\prime}$. By definition, $Z(\mathbf{t})$ is supported on $\mathcal{S}^{\prime}$, hence $Z(\mathbf{t}) \in \mathbb{Z}\left[\left[\mathcal{S}^{\prime}\right]\right]$. It has a natural decomposition $Z(\mathbf{t})=\sum_{h \in H} Z_{h}(\mathbf{t})$, where $Z_{h}(\mathbf{t})=\sum_{\left[l^{\prime}\right]=h} z\left(l^{\prime}\right) \mathbf{t}^{l^{\prime}}$. (Though the exponents of $\mathbf{t}^{l^{\prime}}$ might be rational, that is, $Z(\mathbf{t}) \in \mathbb{Z}\left[\left[t_{1}^{1 / d}, \ldots, t_{|\mathcal{V}|}^{1 / d}\right]\right]$, where $d=\operatorname{det}(\Gamma)$, the right hand side of (2.2.1) still will be called 'rational function', and $\sum_{l^{\prime}} z\left(l^{\prime}\right) \mathbf{t}^{l^{\prime}}$ a 'series'.)

\subsection{Analytic invariants}

In this manuscript we focus mainly on the structure of the Picard group and the holomorphic line bundles on $\widetilde{X}$. The group $\operatorname{Pic}(\tilde{X}):=H^{1}\left(\widetilde{X}, \mathcal{O}_{\widetilde{X}}^{*}\right)$ of isomorphism classes of holomorphic line bundles on $\tilde{X}$ appears in the exact sequence

$$
0 \rightarrow \operatorname{Pic}^{0}(\tilde{X}) \rightarrow \operatorname{Pic}(\tilde{X}) \stackrel{c_{1}}{\longrightarrow} L^{\prime} \rightarrow 0
$$

where $c_{1}$ denotes the first Chern class. Here $\operatorname{Pic}^{0}(\tilde{X})=H^{1}\left(\widetilde{X}, \mathcal{O}_{\tilde{X}}\right) \simeq \mathbb{C}^{p_{g}}$, where $p_{g}$ is the geometric genus of $(X, o) .(X, o)$ is called rational if $p_{g}(X, o)=0$. Artin in $[2,3]$ characterized rationality topologically via the graphs; such graphs are called 'rational'. By this criterion, $\Gamma$ is rational if and only if $\chi(l) \geq 1$ for any effective nonzero cycle $l \in L_{>0}$. Here $\chi(l)=-\left(l, l-Z_{K}\right) / 2$, where $Z_{K} \in L^{\prime}$ is the (anti)canonical cycle identified by adjunction formulae $\left(-Z_{K}+E_{v}, E_{v}\right)+2=0$ for all $v$.

The epimorphism $c_{1}$ admits a unique group homomorphism section $l^{\prime} \mapsto s\left(l^{\prime}\right) \in$ $\operatorname{Pic}(\widetilde{X})$, which extends the natural section $l \mapsto \mathcal{O}_{\widetilde{X}}(l)$ valid for integral cycles $l \in L$, and such that $c_{1}\left(s\left(l^{\prime}\right)\right)=l^{\prime}[35,48]$. We call $s\left(l^{\prime}\right)$ the natural line bundle on $\widetilde{X}$ with Chern class $l^{\prime}$. By its definition, $\mathcal{L}$ is natural if and only if some power $\mathcal{L}^{\otimes n}$ of it has the form $\mathcal{O}_{\widetilde{X}}(l)$ for some $l \in L$.

Natural line bundles appear in the presence of coverings as well. Indeed, let $\pi:\left(X_{a b}, o\right) \rightarrow(X, o)$ be the universal abelian covering of $(X, o)$ (associated with the homomorphism $\pi_{1}(M) \rightarrow H_{1}(M)=H$ ). This is a normal surface singularity endowed with the finite map $\pi$, which is a regular $H$-covering above $X \backslash\{o\}$ and $\pi^{-1}(o)=\{o\}$. The group $H$ acts on $\left(X_{a b}, o\right)$, the action is free off $o$. [At the level of the links it is the regular topological universal abelian covering.] Let $\tilde{\pi}: \widetilde{X_{a b}} \rightarrow \widetilde{X}$ be the (normalized) pullback of $\pi$ by the resolution $\phi: \widetilde{X} \rightarrow X$, in this way $\widetilde{X_{a b}} \rightarrow X_{a b}$ is a partial resolution. Then the Galois group $H$ acts on $\tilde{\pi}_{*}\left(\mathcal{O}_{\widetilde{X_{a b}}}\right)$, whose eigensheaves are $\tilde{\pi}_{*}\left(\mathcal{O}_{\widetilde{X_{a b}}}\right)=\oplus_{h \in H} s\left(-r_{h}\right)$ [35]. Hence, in this way, one recovers all the natural 
line bundles with Chern classes in the open-closed cube. Those with arbitrary Chern classes satisfy $s\left(-l-r_{h}\right)=\mathcal{O}_{\widetilde{X}}(-l) \otimes s\left(-r_{h}\right)$ for certain $l \in L$.

In the sequel we write uniformly $\mathcal{O}_{\widetilde{X}}\left(l^{\prime}\right)$ for $s\left(l^{\prime}\right)$.

Since the branched loci of $\tilde{\pi}: \widetilde{X_{a b}} \rightarrow \tilde{X}$ is included in the normal crossing divisor $E, \widetilde{X_{a b}}$ might have only cyclic quotient singularities. Hence $p_{g}\left(X_{a b}, o\right)=$ $h^{1}\left(\mathcal{O}_{\widetilde{X_{a b}}}\right)$ and $H^{1}\left(\mathcal{O}_{\widetilde{X_{a b}}}\right)=\oplus_{h} H^{1}\left(\widetilde{X}, \mathcal{O}_{\widetilde{X}}\left(-r_{h}\right)\right)$. The dimensions $p_{g}\left(X_{a b}, o\right)_{h}:=$ $h^{1}\left(\widetilde{X}, \mathcal{O}_{\widetilde{X}}\left(-r_{h}\right)\right)(h \in H)$ are called the equivariant geometric genera of $(X, o)$. Clearly, $\sum_{h} p_{g}\left(X_{a b}, o\right)_{h}=p_{g}\left(X_{a b}, o\right)$ and $p_{g}\left(X_{a b}, o\right)_{0}=p_{g}(X, o)$.

2.3.2 Similarly, if $Z \in L_{>0}$ is an effective non-zero integral cycle supported by $E$, and $\mathcal{O}_{Z}^{*}$ denotes the sheaf of units of $\mathcal{O}_{Z}$, then $\operatorname{Pic}(Z)=H^{1}\left(Z, \mathcal{O}_{Z}^{*}\right)$ is the group of isomorphism classes of invertible sheaves on $Z$. It appears in the exact sequence

$$
0 \rightarrow \operatorname{Pic}^{0}(Z) \rightarrow \operatorname{Pic}(Z) \stackrel{c_{1}}{\longrightarrow} L^{\prime}(|Z|) \rightarrow 0
$$

where $\operatorname{Pic}^{0}(Z)=H^{1}\left(Z, \mathcal{O}_{Z}\right)$. Here and in the sequel, $L(|Z|)$ denotes the sublattice of $L$ generated by the base elements $E_{v} \subset|Z|$, and $L^{\prime}(|Z|)$ is its dual lattice.

If $Z_{2} \geq Z_{1}$ then there are natural restriction maps (for simplicity we denote all of them by the same symbol $r), \operatorname{Pic}(\widetilde{X}) \rightarrow \operatorname{Pic}\left(Z_{2}\right) \rightarrow \operatorname{Pic}\left(Z_{1}\right)$. Similar restrictions are defined at $\mathrm{Pic}^{0}$ level too. These restrictions are homomorphisms of the exact sequences (2.3.1) and (2.3.3):

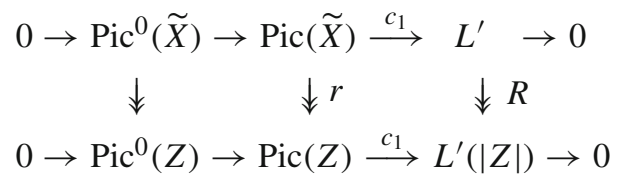

Furthermore, for any $l^{\prime} \in L^{\prime}$ we define a line bundle in $\operatorname{Pic}(Z)$ by $r\left(s\left(l^{\prime}\right)\right)=$ $\left.\mathcal{O}_{\widetilde{X}}\left(l^{\prime}\right)\right|_{Z}$, and we call them restricted natural line bundles on $Z$. They satisfies $c_{1}\left(r\left(s\left(l^{\prime}\right)\right)\right)=R\left(l^{\prime}\right)$.

We also use the notations $\operatorname{Pic}^{l^{\prime}}(\tilde{X}):=c_{1}^{-1}\left(l^{\prime}\right) \subset \operatorname{Pic}(\tilde{X})$ and $\operatorname{Pic}^{R\left(l^{\prime}\right)}(Z):=$ $c_{1}^{-1}\left(R\left(l^{\prime}\right)\right) \subset \operatorname{Pic}(Z)$ respectively. Multiplications by $\mathcal{O}_{\widetilde{X}}\left(-l^{\prime}\right)$ and by $\left.\mathcal{O}_{\widetilde{X}}\left(-l^{\prime}\right)\right|_{Z}$ provide natural (affine space) isomorphisms $\operatorname{Pic}^{l^{\prime}}(\widetilde{X}) \rightarrow \operatorname{Pic}^{0}(\widetilde{X})$ and $\operatorname{Pic}^{R\left(l^{\prime}\right)}(Z) \rightarrow$ $\operatorname{Pic}^{0}(Z)$.

Here an important warning is appropriate. If $\tilde{X}^{\prime}$ is a small connected neighbourhood of some exceptional curves $\cup_{v \in \mathcal{V}^{\prime}} E_{v}, \mathcal{V}^{\prime} \subset \mathcal{V}$, then similarly as for $\widetilde{X}$, but now starting with the invariants of $\widetilde{X}^{\prime}$, one can define the natural line bundles $\mathcal{O}_{\widetilde{X}^{\prime}}\left(l^{\prime}\right)$ for any $l^{\prime} \in L^{\prime}\left(\mathcal{V}^{\prime}\right)$. However, for $l^{\prime} \in L^{\prime}$, in general, if $\mathcal{V}^{\prime} \neq \mathcal{V}$ then $\left.\mathcal{O}_{\tilde{X}^{\prime}}\left(l^{\prime}\right)\right|_{\tilde{X}^{\prime}} \neq \mathcal{O}_{\tilde{X}^{\prime}}\left(R\left(l^{\prime}\right)\right)$, though both line bundles have the same Chern class (here $R$ is the restriction). That is, $\left.\mathcal{O}_{\widetilde{X}}\left(l^{\prime}\right)\right|_{\tilde{X}^{\prime}}$ in general is not the intrinsic natural line bundle of $\widetilde{X}^{\prime}$.

Similarly, for any cycle $Z$ one can define the (intrinsic) natural line bundles of $Z$ by group section of (2.3.3) by similar properties as the natural line bundles of $\widetilde{X}$ are defined. If $|Z|=E$ then they agree with the restrictions $\left.\mathcal{O}_{\widetilde{X}}\left(l^{\prime}\right)\right|_{Z}$. However, if $|Z| \neq E$ then it can happen that $\left.\mathcal{O}_{\tilde{X}}\left(l^{\prime}\right)\right|_{Z}$ is not natural on $Z$. This explains the use of the terminology 'restricted natural line bundle' for $\left.\mathcal{O}_{\widetilde{X}}\left(l^{\prime}\right)\right|_{Z}$ : they are always 
restriction from the $\widetilde{X}$-level. In this article we use only such natural line bundles. In order to simplify the notations we will also write $\mathcal{O}_{Z}\left(l^{\prime}\right):=\left.\mathcal{O}_{\widetilde{X}}\left(l^{\prime}\right)\right|_{Z}, l^{\prime} \in L^{\prime}$.

For any line bundle $\mathcal{L} \in \operatorname{Pic}(\widetilde{X})$ we also write $\mathcal{L}\left(l^{\prime}\right):=\mathcal{L} \otimes \mathcal{O}_{\widetilde{X}}\left(l^{\prime}\right)$.

2.3.5 One of our main interest is to understand the stratification $\{\mathcal{L} \in \operatorname{Pic}(\tilde{X})$ : $\left.h^{1}(\mathcal{L})=k\right\}_{k \in \mathbb{Z}_{\geq 0}}$ of $\operatorname{Pic}(\tilde{X})$. In the literature about $h^{1}(\mathcal{L})$-for arbitrary $\mathcal{L}$-very little is known. However, about the natural line bundles (of some special analytic structures $(X, o))$ recently several results were proved, see e.g. [7,8,36-38]. Since some of these facts are used in several examples and play key role in the general presentation we review them in the next subsection.

2.3.6 The analytic multivariable Poincaré series is defined as follows [37], see also [7,8]. For every $\mathcal{L} \in \operatorname{Pic}(\tilde{X})$ (respectively, for $Z \geq E$ and $\mathcal{L} \in \operatorname{Pic}(Z)$ ) one defines

$$
\begin{aligned}
p_{\mathcal{L}} & :=\sum_{I \subset \mathcal{V}}(-1)^{|I|+1} \operatorname{dim} \frac{H^{0}(\tilde{X}, \mathcal{L})}{H^{0}\left(\widetilde{X}, \mathcal{L}\left(-E_{I}\right)\right)} \text { and } \\
p_{Z, \mathcal{L}} & :=\sum_{I \subset \mathcal{V}}(-1)^{|I|+1} \operatorname{dim} \frac{H^{0}(Z, \mathcal{L})}{H^{0}\left(Z-E_{I}, \mathcal{L}\left(-E_{I}\right)\right)} .
\end{aligned}
$$

For $Z \gg 0$ and $\mathcal{L} \in \operatorname{Pic}(\tilde{X})$ one has $p_{\mathcal{L}}=p_{Z,\left.\mathcal{L}\right|_{Z}}$. If $\left(c_{1}(\mathcal{L}), E_{v}\right)<0$ for some $v \in \mathcal{V}$, then $H^{0}\left(\tilde{X}, \mathcal{L}\left(-E_{I \cup v}\right)\right) \rightarrow H^{0}\left(\widetilde{X}, \mathcal{L}\left(-E_{I}\right)\right)$ is an isomorphism for any $I \not \supset v$ (and similar isomorphism holds for any $Z \geq E$ ), hence

$$
p_{\mathcal{L}}=p_{Z, \mathcal{L}}=0 \quad \text { whenever } c_{1}(\mathcal{L}) \notin-\mathcal{S}^{\prime}
$$

At the level of $\widetilde{X}$ one defines a multivariable series as $P_{\mathcal{L}}(\mathbf{t}):=\sum_{l^{\prime} \in L^{\prime}} p_{\mathcal{L}\left(-l^{\prime}\right)} \mathbf{t}^{l^{\prime}}$. It also has an $H$-decomposition $\sum_{h} P_{\mathcal{L}, h}, P_{\mathcal{L}, h}=\sum_{\left[l^{\prime}\right]=h} p_{\mathcal{L}\left(-l^{\prime}\right)} \mathbf{t}^{t^{\prime}}$, according to the classes $\left[l^{\prime}\right] \in H$ of the exponents of $\mathbf{t}^{l^{\prime}}$. By (2.3.7) it is supported on $c_{1}(\mathcal{L})+\mathcal{S}^{\prime}$. We write $P(\mathbf{t}):=P_{\mathcal{O}_{\widetilde{X}}}(\mathbf{t})=\sum_{l^{\prime}} p_{\mathcal{O}_{\widetilde{X}}\left(-l^{\prime}\right)} \mathbf{t}^{l^{\prime}}$.

The first cohomology of the natural line bundles and the series $P(\mathbf{t})$ are linked by the following identity proved in [37]: for any $l \in L$ one has

$$
h^{1}\left(\tilde{X}, \mathcal{O}\left(-r_{h}-l\right)\right)=-\sum_{a \in L, a \nsupseteq 0} p_{\mathcal{O}\left(-r_{h}-l-a\right)}+p_{g}\left(X_{a b}, o\right)_{h}+\chi(l)-\left(l, r_{h}\right) .
$$

2.3.9 Recently there is an intense activity in the comparison of the analytic invariant $P(\mathbf{t})$ and the topological $Z(\mathbf{t})$ (their coincidence imply e.g. the so-called SeibergWitten Invariant Conjecture [37,38]). For the equality of $P(\mathbf{t})$ and $Z(\mathbf{t})$ for certain families singularities (rational, weighted homogeneous, splice quotient) see e.g. [7,8, 36,37] and the references therein.

We emphasize that in the previous results in the literature the main goal mostly was to characterize for special ('nice') analytic structures the sheaf-theoretical invariants $h^{1}(\mathcal{L})$ topologically, and those methods were applicable only for natural line bundles $\mathcal{L}$. In the present note our goal is to treat $h^{1}(\mathcal{L})$ for any line bundle and for any analytic structure. 


\subsection{Notations}

In the body of the article we will present several examples. In them we will use the following standard notations. We will write $Z_{\min } \in L$ for the minimal (or fundamental) cycle of Artin, which is the minimal non-zero cycle of $\mathcal{S}^{\prime} \cap L[2,3]$. Yau's maximal ideal cycle $Z_{\text {max }} \in L$ is the divisorial part of the pullback of the maximal ideal $\mathfrak{m}_{X, o} \subset \mathcal{O}_{X, o}$, i.e. $\phi^{*} \mathfrak{m}_{X, o} \cdot \mathcal{O}_{\tilde{X}}=\mathcal{O}_{\widetilde{X}}\left(-Z_{\max }\right) \cdot \mathcal{I}$, where $\mathcal{I}$ is an ideal sheaf with 0 -dimensional support [59]. In general $Z_{\text {min }} \leq Z_{\max }$. $Z_{\text {min }}$ can be found by Laufer's algorithm [25]. This algorithm also shows that $h^{0}\left(\mathcal{O}_{Z_{\text {min }}}\right)=1$, hence $h^{1}\left(\mathcal{O}_{Z_{\text {min }}}\right)=1-\chi\left(Z_{\text {min }}\right)$ is topological.

\section{Effective Cartier divisors}

3.1 For any $Z \in L_{>0}$ let $\mathrm{ECa}(Z)$ be the (moduli) space of analytic effective Cartier divisors on $Z$. Their supports are zero-dimensional in $E$. Taking the class of a Cartier divisor provides the Abel map $c: \mathrm{ECa}(Z) \rightarrow \operatorname{Pic}(Z)$. Let $\mathrm{ECa}^{l^{\prime}}(Z)$ be the set of effective Cartier divisors with Chern class $l^{\prime} \in L^{\prime}(|Z|)$, that is, $\mathrm{ECa}^{l^{\prime}}(Z):=c^{-1}\left(\operatorname{Pic}^{l^{\prime}}(Z)\right)$. Sometimes we denote the restriction of $c$ by $c^{l^{\prime}}: \mathrm{ECa}^{l^{\prime}}(Z) \rightarrow \operatorname{Pic}^{l^{\prime}}(Z), l^{\prime} \in L^{\prime}(|Z|)$. It is also convenient to use the simplified notation $\mathrm{ECa}^{l^{\prime}}(Z):=\mathrm{ECa}^{R\left(l^{\prime}\right)}(Z)$ and $\operatorname{Pic}^{l^{\prime}}(Z):=\operatorname{Pic}^{R\left(l^{\prime}\right)}(Z)$ for any $l^{\prime} \in L^{\prime}$ (where $R: L^{\prime} \rightarrow L^{\prime}(|Z|)$ is the restriction as above).

For any $Z_{2} \geq Z_{1}>0$ (and $l^{\prime} \in L^{\prime}$ ) one has the commutative diagram

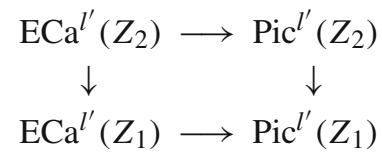

Regarding the existence of $\operatorname{ECa}(Z)$ and the Abel map we note the following. First, by a theorem of Artin $[4,3.8]$, there exists an affine algebraic variety $Y$ and a point $y \in$ $Y$ such that $(Y, y)$ and $(X, o)$ have isomorphic formal completions. Then, according to Hironaka [18], $(Y, y)$ and $(X, o)$ are analytically isomorphic. In particular, we can regard $Z$ as a projective algebraic scheme. In this algebraic context, $\mathrm{ECa}^{l^{\prime}}(Z)$ as an algebraic variety-together with the algebraic Abel map was constructed by Grothendieck [16], see e.g. the article of Kleiman [20] with several comments and citations and the book of Mumford for curves on algebraic surfaces [31]. (Recall that $\operatorname{Pic}(Z) \simeq \mathbb{C}^{h^{1}\left(\mathcal{O}_{Z}\right)}$ is an affine space.) In particular,

$$
c: \operatorname{ECa}(Z) \rightarrow \operatorname{Pic}(Z) \text { is algebraic. }
$$

(For concrete charts of $\mathrm{ECa}^{l^{\prime}}(Z)$ see e.g. the proof of theorem 3.1.10 and for the Abel map in concrete charts see Sect. 7.) Though these spaces are identified by the general theory, in the body of this note we verify directly several properties of them in order to illuminate the peculiarities of the present situation, e.g. we discuss the smoothness and the dimension of $\mathrm{ECa}^{l^{\prime}}(Z)$ and the structure of the fibers of the Abel map: the 
related numerical invariants will be crucial in the further discussions. Doing this we develop several special properties of the Abel map in the language of invariants of normal surface singularities; these connections will be exploited deeply.

We write $\operatorname{ECa}(\tilde{X})$ for the set of effective Cartier divisors on $\widetilde{X}$.

3.1.2 Let us fix $Z \in L, Z>0$. As usual, we say that $\mathcal{L} \in \operatorname{Pic}^{l^{\prime}}(Z)$ has no fixed components if

$$
H^{0}(Z, \mathcal{L})_{\text {reg }}:=H^{0}(Z, \mathcal{L}) \backslash \bigcup_{E_{v} \subset|Z|} H^{0}\left(Z-E_{v}, \mathcal{L}\left(-E_{v}\right)\right)
$$

is non-empty. Here the inclusion of $H^{0}\left(Z-E_{v}, \mathcal{L}\left(-E_{v}\right)\right)$ into $H^{0}(Z, \mathcal{L})$ is given by the long cohomological exact sequence associated with $0 \rightarrow \mathcal{L}\left(-E_{v}\right) \rightarrow \mathcal{L} \rightarrow$ $\left.\mathcal{L}\right|_{E_{v}} \rightarrow 0$, and it represents the subspace of sections, whose fixed components contain $E_{v}$.

Note that $H^{0}(Z, \mathcal{L})$ is a module over the algebra $H^{0}\left(\mathcal{O}_{Z}\right)$, hence one has a natural

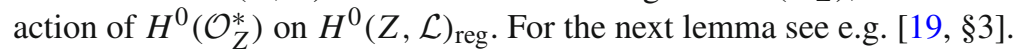

Lemma 3.1.4 $\mathcal{L} \in \operatorname{Pic}^{l^{\prime}}(Z)$ is in the image of $c^{l^{\prime}}: \mathrm{ECa}^{l^{\prime}}(Z) \rightarrow \operatorname{Pic}^{l^{\prime}}(Z)$ if and only if $H^{0}(Z, \mathcal{L})_{\text {reg }} \neq \emptyset$. In this case, $c^{-1}(\mathcal{L})=H^{0}(Z, \mathcal{L})_{\text {reg }} / H^{0}\left(\mathcal{O}_{Z}^{*}\right)$.

In the next discussion we assume $Z \geq E$ basically imposed by the easement of the presentation; everything can be adopted for any $Z>0$, see e.g. 4.1.4 or 5.1.

Note that $H^{0}(Z, \mathcal{L})_{\text {reg }} \neq \varnothing \Rightarrow H^{0}\left(\left.\mathcal{L}\right|_{E_{v}}\right) \neq 0 \forall v \Rightarrow\left(l^{\prime}, E_{v}\right) \geq 0 \forall v \Rightarrow$ $l^{\prime} \in-\mathcal{S}^{\prime}$. Conversely, if $l^{\prime}=-\sum_{v} m_{v} E_{v}^{*} \in-\mathcal{S}^{\prime}$ (for certain $m_{v} \in \mathbb{Z}_{\geq 0}$ ), and $l^{\prime} \neq 0$, then one can construct for each $E_{v}$ cuts (local complex discs considered as reduced divisors) in $\widetilde{X}$ intersecting $E_{v}$ in a generic point and having with it intersection multiplicity $m_{v}$. Since $l^{\prime} \neq 0$ their collection is nonempty, and it provides elements in $\mathrm{ECa}^{l^{\prime}}(\widetilde{X})$ and $\mathrm{ECa}^{l^{\prime}}(Z)$ respectively (the second one by restriction). However, this collection is empty whenever $l^{\prime}=0$, hence this special case needs slightly more attention. By definition we declare that $\mathrm{ECa}^{0}(Z)$ is a space consisting of a point (what we can call the 'empty divisor'), $\mathrm{ECa}^{0}(Z)=\{\emptyset\}$, and $c^{0}: \mathrm{ECa}^{0}(Z) \rightarrow \operatorname{Pic}^{0}(Z)$ is defined as $c^{0}(\emptyset)=\mathcal{O}_{Z}$. Since for $l^{\prime}=0$ any section from $H^{0}(Z, \mathcal{L})_{\text {reg }}$ trivializes $\mathcal{L}$, one has:

$$
H^{0}(Z, \mathcal{L})_{\text {reg }} \neq \emptyset \Leftrightarrow \mathcal{L}=\mathcal{O}_{Z} \Leftrightarrow \mathcal{L} \in \operatorname{im}\left(c^{0}\right) \quad\left(l^{\prime}=0\right) .
$$

Therefore, the above discussions combined provide

$$
\mathrm{ECa}^{l^{\prime}}(Z) \neq \varnothing \Leftrightarrow l^{\prime} \in-\mathcal{S}^{\prime}
$$

The action of $H^{0}\left(\mathcal{O}_{Z}^{*}\right)$ can be analysed quite explicitly. Note that from the exact sequence

$$
0 \rightarrow H^{0}\left(\mathcal{O}_{Z-E}(-E)\right) \rightarrow H^{0}\left(\mathcal{O}_{Z}\right) \stackrel{r_{E}}{\longrightarrow} H^{0}\left(\mathcal{O}_{E}\right)=\mathbb{C} \rightarrow 0
$$

one gets that $H^{0}\left(\mathcal{O}_{Z}^{*}\right)=r_{E}^{-1}\left(\mathbb{C}^{*}\right)=H^{0}\left(\mathcal{O}_{Z}\right) \backslash H^{0}\left(\mathcal{O}_{Z-E}(-E)\right)$. In particular, $H^{0}\left(\mathcal{O}_{Z}^{*}\right)$, as algebraic variety, has the dimension of the vector space $H^{0}\left(\mathcal{O}_{Z}\right)$, 
$\mathbb{P} H^{0}\left(\mathcal{O}_{Z}^{*}\right):=H^{0}\left(\mathcal{O}_{Z}^{*}\right) / \mathbb{C}^{*}$ as algebraic variety is isomorphic with $H^{0}\left(\mathcal{O}_{Z-E}(-E)\right)$, and $H^{0}(Z, \mathcal{L})_{\text {reg }} / H^{0}\left(\mathcal{O}_{Z}^{*}\right)=\mathbb{P} H^{0}(Z, \mathcal{L})_{\text {reg }} / \mathbb{P} H^{0}\left(\mathcal{O}_{Z}^{*}\right)$. (Here, again, $\mathbb{P} H^{0}(Z, \mathcal{L})_{\text {reg }}$ by definition denotes $H^{0}(Z, \mathcal{L})_{\text {reg }} / \mathbb{C}^{*}$.)

Lemma 3.1.7 Assume that $H^{0}(Z, \mathcal{L})_{\text {reg }} \neq \emptyset$. Then

(a) the action of $H^{0}\left(\mathcal{O}_{Z}^{*}\right)$ on $H^{0}(Z, \mathcal{L})_{\text {reg }}$ is algebraic, free and proper;

(b) $\mathbb{P} H^{0}(Z, \mathcal{L})_{\text {reg }}$ over $\mathbb{P} H^{0}(Z, \mathcal{L})_{\text {reg }} / \mathbb{P} H^{0}\left(\mathcal{O}_{Z}^{*}\right)$ is a principal affine bundle.

Hence, the fiber $c^{-1}(\mathcal{L}), \mathcal{L} \in \operatorname{im}\left(c^{l^{\prime}}\right)$, is an irreducible quasiprojective smooth variety of dimension

$$
h^{0}(Z, \mathcal{L})-h^{0}\left(\mathcal{O}_{Z}\right)=\left(l^{\prime}, Z\right)+h^{1}(Z, \mathcal{L})-h^{1}\left(\mathcal{O}_{Z}\right)
$$

Proof For $s \in H^{0}(Z, \mathcal{L})_{\text {reg }}$ the multiplication by $s, \mathcal{O}_{Z} \stackrel{\cdot s}{\longrightarrow} \mathcal{L}$, is injective, hence induces injections $H^{0}\left(\mathcal{O}_{Z}\right) \stackrel{\cdot s}{\longrightarrow} H^{0}(\mathcal{L})$ and $H^{0}\left(\mathcal{O}_{Z}^{*}\right) \stackrel{\cdot s}{\longrightarrow} H^{0}(\mathcal{L})_{\text {reg. }}$. Hence the action is free. Next we prove that the action of $\mathbb{P} H^{0}\left(\mathcal{O}_{Z}^{*}\right)$ on $\mathbb{P} H^{0}(Z, \mathcal{L})_{\text {reg }}$ is proper.

Introduce hermitian metrics in both $H^{0}\left(\mathcal{O}_{Z}\right)$ and $H^{0}(Z, \mathcal{L})$. Write $H^{0}:=$ $H^{0}\left(\mathcal{O}_{Z-E}(-E)\right)$ in $H^{0}\left(\mathcal{O}_{Z}\right)$ and choose $h^{\perp}$ with $H^{0}\left(\mathcal{O}_{Z}\right)=H^{0} \oplus \mathbb{C}\left\langle h^{\perp}\right\rangle$. Set also $B:=\cap_{v} H^{0}\left(Z-E_{v}, \mathcal{L}\left(-E_{v}\right)\right) \subset H^{0}(Z, \mathcal{L})$ and let $B^{\perp}$ be its unitary complement in $H^{0}(Z, \mathcal{L})$. Note that $H^{0}(Z, \mathcal{L}) \backslash B$ is also stable with respect to the action of $H^{0}\left(\mathcal{O}_{Z}^{*}\right)=B \oplus \mathbb{C}\left\langle h^{\perp}\right\rangle \backslash B \oplus 0$. Since $H^{0}(Z, \mathcal{L})_{\text {reg }}$ is open in $H^{0}(Z, \mathcal{L}) \backslash B$, it is enough to show that $H^{0}\left(\mathcal{O}_{Z}^{*}\right)$ acts properly on $H^{0}(Z, \mathcal{L}) \backslash B$. Fix $K$ compact in $H^{0}(Z, \mathcal{L}) \backslash B$ and let $K^{\prime}$ be its lift to the unit sphere of $H^{0}(Z, \mathcal{L})$. We need to show that if $h=h^{0}+h^{\perp} \in H^{0} \oplus \mathbb{C}\left\langle h^{\perp}\right\rangle$ and $\left|h^{0}\right| \rightarrow \infty$, and $k \in K^{\prime}$, then the components $(h k)_{1}+(h k)_{2} \in B \perp B^{\perp}$ of $h k$ satisfy $\left|(h k)_{1}\right| /\left|(h k)_{2}\right| \rightarrow \infty$. For this note the following facts.

First, $H^{0} \cdot H^{0}(Z, \mathcal{L}) \subset B$, hence $\left(h^{0} k\right)_{2}=0$. Next, since $K^{\prime}$ is compact, $\left|\left(h^{\perp} k\right)_{1}\right|$ and $\left|\left(h^{\perp} k\right)_{2}\right|$ are bounded from above. Finally, since $h^{0} k \neq 0$, for any $h^{0}$ in the unit sphere, the set $\left\{\left|h^{0} k\right|\right\}_{k}$ is bounded from below by a positive number. Hence, whenever $\left|h^{0}\right| \rightarrow \infty$ one also has

$$
\left|(h k)_{1}\right| /\left|(h k)_{2}\right|=\left|\left(h^{\perp} k\right)_{1}+\right| h^{0}\left|\cdot\left(\frac{h^{0}}{\left|h^{0}\right|} \cdot k\right)\right| /\left|\left(h^{\perp} k\right)_{2}\right| \rightarrow \infty .
$$

(a) implies (b) (since $\mathbb{P} H^{0}\left(\mathcal{O}_{Z}^{*}\right) \simeq H^{0}$ is an affine space) and the equality in (3.1.8) follows from Riemann-Roch formula.

Example 3.1.9 Assume that $(X, o)$ is rational, and $l^{\prime} \in-\mathcal{S}^{\prime}$. Then $\operatorname{Pic}^{l^{\prime}}(Z)=0$, hence if $c_{1}(\mathcal{L})=l^{\prime}$ then $\mathcal{L}=\mathcal{O}\left(l^{\prime}\right)$. Furthermore, $\mathcal{L}$ is basepoint free [27, Th. 12.1]. Thus $\mathrm{ECa}^{l^{\prime}}(Z)=H^{0}(Z, \mathcal{L})_{\text {reg }} / H^{0}\left(\mathcal{O}_{Z}^{*}\right)$ and since the action of $H^{0}\left(\mathcal{O}_{Z}^{*}\right)$ is free (cf. 3.1.7), $\mathrm{ECa}^{l^{\prime}}(Z)$ is smooth. Since $h^{1}(Z, \mathcal{L})=h^{1}\left(\mathcal{O}_{Z}\right)=0$ (cf. [27,34]), the dimension of $\mathrm{ECa}^{l^{\prime}}(Z)$ is $\left(l^{\prime}, Z\right)$ (use (3.1.8)). Furthermore, its topological Euler characteristic is $\chi_{\text {top }}\left(\mathrm{ECa}^{l^{\prime}}(Z)\right)=\chi_{\text {top }}\left(\mathbb{P} H^{0}(Z, \mathcal{L})_{\text {reg }}\right)$, which is the coefficient $z\left(-l^{\prime}\right)$ of the multivariable series $Z(\mathbf{t})$ by $[8,36,37]$.

These facts generalize as follows. 
Theorem 3.1.10 If $l^{\prime} \in-\mathcal{S}^{\prime}$ then the following facts hold.

(1) $\mathrm{ECa}^{l^{\prime}}(Z)$ is a smooth complex (irreducible) variety of dimension $\left(l^{\prime}, Z\right)$.

(2) The topological Euler characteristic of $\mathrm{ECa}^{l^{\prime}}(Z)$ is $z\left(-l^{\prime}\right)$. In fact, the natural restriction $r: \mathrm{ECa}^{l^{\prime}}(Z) \rightarrow \mathrm{ECa}^{l^{\prime}}(E)$ is a locally trivial fiber bundle with fiber isomorphic to an affine space. Hence, the homotopy type of $\mathrm{ECa}^{l^{\prime}}(Z)$ is independent of the choice of $Z$ and it depends only on the topology of $(X, o)$.

(3) $r: \mathrm{ECa}^{l^{\prime}}\left(Z_{2}\right) \rightarrow \mathrm{ECa}^{l^{\prime}}\left(Z_{1}\right)$ is surjective for any $Z_{2} \geq Z_{1}$.

Proof As we already said in the first paragraphs of $3.1, \mathrm{ECa}^{l^{\prime}}(Z)$ is an algebraic variety, cf. [16,20]. We need to construct in the neighbourhood of each Cartier divisor a smooth chart.

First assume that $Z=E$. Then $\mathrm{ECa}^{l^{\prime}}(E)$ is independent of the self-intersections $E_{v}^{2}$, hence (keeping the analytic type of $E$, but) modifying the self-intersections into very negative integers, we can assume that the singularity is rational. In this modified case, $\mathrm{ECa}^{l^{\prime}}(E)=\mathbb{P}\left(H^{0}\left(E, \mathcal{O}\left(l^{\prime}\right)\right)_{\text {reg }}\right)$, see Example 3.1.9. Note that $H^{0}\left(E, \mathcal{O}\left(l^{\prime}\right)\right)_{\text {reg }}$ is also independent of the self-intersection numbers, hence, in any case, $\mathrm{ECa}^{l^{\prime}}(E)=\mathbb{P}\left(H^{0}\left(E, \mathcal{O}\left(l^{\prime}\right)\right)_{\text {reg }}\right)$. In particular, $\mathrm{ECa}^{l^{\prime}}(E)$ is smooth, irreducible and with the required dimension and Euler characteristic, cf. Example 3.1.9.

Let us provide some local charts of $\mathrm{ECa}^{l^{\prime}}(E)$. Fix $D \in \mathrm{ECa}^{l^{\prime}}(E)$ with support $\left\{p_{i}\right\}_{i} \subset E$.

If $p_{i} \in E_{v}$ is a smooth point of $E$, then there exists a local neighbourhood $U_{i}$ of $p_{i}$ in $\widetilde{X}$ with local coordinates $(x, y)$ such that $\{x=0\}=E \cap U_{i}$ and $D$ in $U_{i}$ is represented by the local Cartier equation $\left\{y^{m}\right\}$ for some $m \in \mathbb{Z}_{>0}$. Then a local neighbourhood $\mathcal{U}_{i}(E)$ of the divisor $\left\{y^{m}\right\}$ in $\mathrm{ECa}^{-m E_{v}^{*}}(E)$ is given by local Cartier divisors $\left\{y^{m}+f(y)\right\}$, where $f \in \mathcal{O}\left(E \cap U_{i}\right)$ is a small perturbation of the zero function, modulo the multiplicative action of $\mathcal{O}^{*}\left(E \cap U_{i}\right)$. Multiplying $y^{m}$ by $1+a_{k} y^{k}$ we get that perturbation of type $y^{m}+\sum_{k \geq 0} a_{k} y^{k+m}$ constitute the orbit of $y^{m}$ (or, differently said, $\sum_{k \geq 0} a_{k} y^{k+m}$ is the tangent space of the orbit). Therefore, the smooth transversal slice to this orbit $\left(a_{i}\right)_{0 \leq i<m} \mapsto\left\{y^{m}+\sum_{i<m} a_{i} y^{i}\right\}\left(\left|a_{i}\right| \ll 1\right)$ provides a smooth chart $\mathcal{U}_{i}(E)$ of dimension $m=\left(-m E_{v}^{*}, E\right)$. Here, $-m E_{v}^{*}$ is the local contribution in the Chern class $l^{\prime}$.

Similarly, if $p_{i}=E_{u} \cap E_{v}$, then there exists a neighbourhood $U_{i}$ of $p_{i}$ in $\widetilde{X}$ with local coordinates $(x, y)$ such that $\{x=0\}=U_{i} \cap E_{v}$ and $\{y=0\}=U_{i} \cap E_{u}$, and $D$ in $U_{i}$ is represented by $\left\{x^{n}+y^{m}\right\}$ for certain $n, m \in \mathbb{Z}_{>0}$. [Indeed, any Cartier divisor in $\mathbb{C}[[x, y]] /(x y) \simeq \mathcal{O}_{E, p_{i}}$ can be represented by a local equation in $U_{i}$ of this type.] Then, a local neighbourhood $\mathcal{U}_{i}(E)$ of $x^{n}+y^{m}$ in $\mathrm{ECa}^{-m E_{v}^{*}-n E_{u}^{*}}(E)$ is given by $\left\{x^{n}+y^{m}+a_{0}+\sum_{i \geq 1} a_{i} x^{i}+\sum_{i \geq 1} b_{i} y^{i}\right\}$ modulo the action of $\mathcal{O}^{*}\left(E \cap U_{i}\right)$. The orbit of this action at $x^{n}+y^{m}$ is $\left\{x^{n}+y^{m}+\sum_{i>n} a_{i} x^{i}+\sum_{i>m} b_{i} y^{i}+\lambda\left(x^{n}+y^{m}\right)\right\}$, it is smooth. A possible smooth slice of it is $\left\{x^{n}+y^{m}+a_{0}+\sum_{i=1}^{n} a_{i} x^{i}+\sum_{i=1}^{m} b_{i} y^{i}\right\} /\left\{a_{n}+b_{m}=0\right\}$, which is of dimension $\left(-m E_{v}^{*}-n E_{u}^{*}, E\right)$ (the local contribution into $\left(l^{\prime}, E\right)$ ).

Products of type $\mathcal{U}(D)=\prod_{i} \mathcal{U}_{i}(E)$ constitute a local neighbourhood of $D$ in $\mathrm{ECa}^{l^{\prime}}(E)$.

Consider now an arbitrary $Z \geq E$ and the restriction $r: \mathrm{ECa}^{l^{\prime}}(Z) \rightarrow \mathrm{ECa}^{l^{\prime}}(E)$. We show that $\operatorname{ECa}^{l^{\prime}}(Z)$ can be covered by open sets of type $r^{-1}\left(\prod_{i} \mathcal{U}_{i}(E)\right)=$ $\prod_{i} r_{i}^{-1}\left(\mathcal{U}_{i}(E)\right)$, where $r_{i}$ is either the restriction $\mathrm{ECa}^{-m E_{v}^{*}}(Z) \rightarrow \mathrm{ECa}^{-m E_{v}^{*}}(E)$ or 
$\mathrm{ECa}^{-m E_{v}^{*}-n E_{u}^{*}}(Z) \rightarrow \mathrm{ECa}^{-m E_{v}^{*}-n E_{u}^{*}}(E)$, and each $r_{i}^{-1}\left(\mathcal{U}_{i}(E)\right)$ is a product of $\mathcal{U}_{i}(E)$ and an affine space.

Indeed, assume first that $p_{i}$ is a smooth point of $E$ as above, $p_{i} \in E_{v}$, and let $N \geq 1$ be the multiplicity of $Z$ along $E_{v}$. Then in $U_{i}$ the local equation of $Z$ is $x^{N}$ and let us fix a Cartier divisor in $r^{-1}\left(\mathcal{U}_{i}(E)\right)$ whose restriction is $y^{m}$, represented by $f:=$ $y^{m}+x g(x, y)$ for some $g \in \mathcal{O}\left(U_{i}\right) /\left(x^{N-1}\right)$, modulo $\mathcal{O}^{*}\left(U_{i}\right) /\left(x^{N}\right)$. Multiplication $f\left(1+a_{i} y^{i} x^{N-1}\right) \equiv f+a_{i} y^{m+i} x^{N-1}$ shows that $f+y^{m} x^{N-1} \mathcal{O}\left(U_{i}\right)\left(\bmod \left(x^{N}\right)\right)$ is in the orbit. Using this fact, and multiplication by $1+a_{i} y^{i} x^{N-2}$ one shows that $f+y^{m} x^{N-2} \mathcal{O}\left(U_{i}\right)\left(\bmod \left(x^{N}\right)\right)$ is also in the orbit. By induction, we get that the orbit is $f+y^{m} \mathcal{O}\left(U_{i}\right)\left(\bmod \left(x^{N}\right)\right)$, and it is smooth. A transversal smooth cut (slice) can be parametrized by the chart $\left\{y^{m}+\sum_{i<N, j<m} a_{i j} x^{i} y^{j}\right\}$, which has dimension $\left(-m E_{v}^{*}, Z\right)=m N$. For $i>0$ the variables $a_{i j}$ can be chosen as affine coordinates.

More conceptually, in this case, multiplication of $f$ by $1+h$ gives $f+f h$ (mod $\left(x^{N}\right)$ ), hence the orbit is identified with $f+\operatorname{ideal}\left(f, x^{N}\right)$, which has a smooth section whose dimension is the codimension of $\operatorname{ideal}\left(f, x^{N}\right)$, that is, the intersection multiplicity $\left(f, x^{N}\right)_{p_{i}}=m N$.

Similar chart can be found in the case of $p_{i}=E_{u} \cap E_{v}$ as well. Let us use the previous notations, let us fix a divisor $f=x^{n}+y^{m}+x y g(x, y)$ whose restriction to $E$ is $x^{n}+y^{m}$, and assume that in $Z$ the multiplicities of $\{x=0\}$ and $\{y=0\}$ are $N$ and $M$. Then the orbit is identified with $f+\operatorname{ideal}\left(f, x^{N} y^{M}\right)$, which has a smooth transversal cut whose dimension is the intersection multiplicity $\left(f, x^{N} y^{M}\right)_{p_{i}}=m N+n M$. The $m N+n M$ coordinates of the cut cannot be chosen canonically. We invite the reader to check that these coordinates can be chosen in such a way that first we choose the $m+n$ (local) coordinates of the reduces part (as above in the case $Z=E$ ) then we can complete them with $m(N-1)+n(M-1)$ affine coordinates.

Taking product we obtain charts of type $\prod_{i} \mathcal{U}_{i}(Z):=r^{-1}\left(\prod_{i} \mathcal{U}_{i}(E)\right)=$ $\left(\prod_{i} \mathcal{U}_{i}(E)\right) \times \mathbb{C}^{\left(l^{\prime}, Z-E\right)}$.

(3) follows from the description of the above charts.

\subsection{The tangent map of $c$. The smoothness of $c^{-1}(\mathcal{L})$}

Assume that $\mathcal{L} \in \operatorname{Pic}^{l^{\prime}}(Z)$ has no fixed components. Fix any $D \in c^{-1}(\mathcal{L}) \subset \mathrm{ECa}^{l^{\prime}}(Z)$, and let $s \in H^{0}(Z, \mathcal{L})$ be the section whose divisor is $D$. Then multiplication by $s$ gives an exact sequence of sheaves

$$
0 \rightarrow \mathcal{O}_{Z} \stackrel{\cdot s}{\longrightarrow} \mathcal{L} \rightarrow \mathcal{O}_{D} \rightarrow 0
$$

Division by $s$ identifies $\mathcal{L}$ by $\mathcal{O}_{Z}(D)$, hence the above exact sequence can be identified with the exact sequence $0 \rightarrow \mathcal{O}_{Z} \rightarrow \mathcal{O}_{Z}(D) \rightarrow \mathcal{O}_{D}(D) \rightarrow 0$ (this is a generalization of the so-called Mittag-Lefler sequence, defined for effective divisors on curves).

We emphasize that $\mathcal{O}_{D}$ is finitely supported. The dimension of $H^{0}\left(\mathcal{O}_{D}\right)$ is $\left(l^{\prime}, Z\right)$.

Proposition 3.2.2 The coboundary homomorphism $\delta_{D}^{1}: H^{0}\left(\mathcal{O}_{D}\right) \rightarrow H^{1}\left(\mathcal{O}_{Z}\right)$ of the cohomological long exacts sequence of (3.2.1) can be identified with the tangent map

$$
T_{D}\left(c^{l^{\prime}}\right): T_{D}\left(E C a^{l^{\prime}}(Z)\right) \rightarrow T_{\mathcal{L}}\left(\operatorname{Pic}^{l^{\prime}}(Z)\right)
$$


of $c^{l^{\prime}}$ at D. Moreover, the Zariski tangent space $T_{D}\left(c^{-1}(\mathcal{L})\right)$ of $c^{-1}(\mathcal{L})$ at $D$ is identified with its kernel, hence (by the cohomological long exact sequence) by $H^{0}(Z, \mathcal{L}) / H^{0}\left(\mathcal{O}_{Z}\right)$. This shows that $\operatorname{dim} T_{D}\left(c^{-1}(\mathcal{L})\right)=\operatorname{dim} c^{-1}(\mathcal{L})$ at any $D \in$ $c^{-1}(\mathcal{L})(c f .(3.1 .8))$, hence $c^{-1}(\mathcal{L})$ is smoothly embedded into $\mathrm{ECa}^{l^{\prime}}(Z)$, and $c^{-1}(\mathcal{L})$, as a subscheme of $\mathrm{ECa}^{l^{\prime}}(Z)$, can be identified with $H^{0}(Z, \mathcal{L})_{\mathrm{reg}} / H^{0}\left(\mathcal{O}_{Z}^{*}\right)$.

This fact reformulated shows that $\delta_{D}^{1}$ induced on $N_{D}\left(c^{-1}(\mathcal{L})\right):=T_{D}\left(E C a^{l^{\prime}}(Z)\right) /$ $T_{D}\left(c^{-1}(\mathcal{L})\right)$, the normal space of $c^{-1}(\mathcal{L}) \subset E C a^{l^{\prime}}$ at $D$, is injective.

Proof See [31, p. 164], or [19, Remark 5.18], or [20, §5].

Corollary 3.2.3 If $\operatorname{dim}\left(\mathrm{ECa}^{l^{\prime}}(Z)\right)=1$ and $c^{l^{\prime}}$ is not constant then $\operatorname{im}\left(c^{l^{\prime}}\right)$ is smooth.

\subsection{The special fibers of $c^{\prime}$}

Though all the fibers of $c^{l^{\prime}}$ are smooth, still we wish to distinguish certain fibers of $c^{l^{\prime}}$ with pathological behaviour. There are several types we can consider.

Definition 3.3.1 (a) $D \in \mathrm{ECa}^{l^{\prime}}(Z)$ is called a critical divisor (point) if $\operatorname{rank}\left(T_{D} c\right)<$ $\operatorname{rank}\left(T_{D_{\text {gen }}} c\right)$, where $D_{\text {gen }} \in \mathrm{ECa}^{l^{\prime}}(Z)$ is a generic divisor. If $\left(c^{l^{\prime}}\right)^{-1}(\mathcal{L})$ contains a critical divisor (point) then $\mathcal{L}$ is a called a critical bundle (value).

(b) We say that $\mathcal{L} \in \operatorname{im}\left(c^{l^{\prime}}\right)$ is $T$-typical ('tangent-map-typical') if the linear subspace $\operatorname{im}\left(T_{D}\left(c^{l^{\prime}}\right)\right) \subset T_{\mathcal{L}} \mathrm{Pic}^{l^{\prime}}(Z)$ is independent of the choice of $D \in c^{-1}(\mathcal{L})$. Otherwise $\mathcal{L}$ is $T$-atypical.

The prototype of a map with a $T$-atypical value is the blowing up $c: B \rightarrow \mathbb{C}^{2}$ at the origin $0 \in \mathbb{C}^{2}$ : then 0 is a $T$-atypical value. For such an example realized by a concrete $c^{l^{\prime}}$ see 3.4.3.

Lemma 3.3.2 For fixed $l^{\prime}$ and $\mathcal{L} \in \operatorname{im}\left(c^{l^{\prime}}\right)$ consider the following properties:

(i) $\mathcal{L}$ is a $T$-atypical value of $c^{l^{\prime}}$,

(ii) $\mathcal{L}$ is a singular point of the closure $\overline{\operatorname{im}\left(c^{l^{\prime}}\right)}$ of the image of $c^{l^{\prime}}$ (where $\overline{\operatorname{im}\left(c^{l^{\prime}}\right)}$ is taken with the reduced structure),

(iii) $\operatorname{dim}\left(\left(c^{l^{\prime}}\right)^{-1}(\mathcal{L})\right)$ is strictly larger the the dimension of the generic fiber of $c^{l^{\prime}}$,

(iv) $\mathcal{L}$ is critical bundle,

(v) any $D \in\left(c^{l^{\prime}}\right)^{-1}(\mathcal{L})$ is a critical divisor.

$\overline{\text { Then }}($ iii $) \Leftrightarrow(i v) \Leftrightarrow(v)$,

(i) $\Rightarrow$ (iii) and

(ii) $\Rightarrow$ (iii).

Proof The equivalences (iii) $\Leftrightarrow$ (iv) $\Leftrightarrow(v)$ follow from Proposition 3.2.2. For $(i) \Rightarrow$ (iii) first notice that $c^{-1}(\mathcal{L})$ is smooth and irreducible, hence it is enough to verify the statement locally at a generic point of $c^{-1}(\mathcal{L})$. On the other hand, if (iii) is not true, that is, if (locally) $\operatorname{rank}\left(T_{D} c\right)=\operatorname{rank}\left(T_{D_{\text {gen }}} c\right)$, then $c$ in that neighbourhood is a fibration, hence (locally) the normal bundle of $c^{-1}(\mathcal{L})$ is a pullback of a vector space $V$, hence (using also Proposition 3.2.2) $\mathrm{im}\left(T_{D}(c)\right.$ ) is constant $V$.

(ii) $\Rightarrow$ (iii). Assume that (iii) is not true, hence, as in the previous case, $\operatorname{rank}\left(T_{D} c\right)=$ $\operatorname{rank}\left(T_{D_{\text {gen }}} c\right)$ for any $D \in c^{-1}(\mathcal{L})$, and $c$ in that neighbourhood is a fibration. $\operatorname{im}(c)$ 
is the image of the quotient space obtained from the total space by collapsing each fibers into a point. But for any $D \in c^{-1}(\mathcal{L})$ the space $N_{D}\left(c^{-1}(\mathcal{L})\right)$ is mapped by $T_{D} c$ injectively onto $\operatorname{im}\left(T_{D} c\right)$, and this image is independent of the choice of $D$ [by the proof $(i) \Rightarrow($ iii $)]$. This shows that, in fact, $\operatorname{im}(c)$ is immersed at $\mathcal{L}$. Since the fiber $c^{-1}(\mathcal{L})$ is connected, $\operatorname{im}(c)$ is in fact embedded. Hence, $\operatorname{im}(c)$ is smooth at $\mathcal{L}$.

Remark 3.3.3 (a) In principle, by general properties of algebraic maps, neither (iii) nor (ii) imply (i) by general nonsense. Indeed, set $Y:=\left\{(x, y, a) \in \mathbb{C}^{3}: y=a x^{2}\right\}$ and consider the projection $p: Y \rightarrow \mathbb{C}^{2}$ induced by $(x, y, a) \mapsto(x, y)$. Then the fiber $p^{-1}(0,0)$ satisfy (iii) but not $(i)$.

Take also $Y:=\mathbb{C}^{*} \times \mathbb{C}$ and consider the map $Y \rightarrow \mathbb{C}^{3}$ given by $(x, y) \mapsto$ $\left(y x, y^{2} x, y^{3} x\right)$. Then the closure of the image is singular at the origin. This value satisfies (ii) and (iii) but not (i).

The implication (iii) $\Rightarrow$ (ii) also fails, in general. E.g., if $c^{l^{\prime}}$ is dominant, and $\mathcal{L} \in \operatorname{im}\left(c^{l^{\prime}}\right)$, then $\mathcal{L}$ is not a distinguished point of the closure of the image even if it is critical, see e.g. 3.4.3. However, examples suggest the following conjectural property: if $c^{l^{\prime}}$ is dominant and some $\mathcal{L} \in \operatorname{Pic}^{l^{\prime}}(Z)$ satisfies (iii) then $\mathcal{L}$ is not an interior point of $\operatorname{im}(c)$.

(b) We wish to emphasize again that $c^{l^{\prime}}$ is not proper. In particular, above a small (relative) neighbourhood in $\operatorname{im}\left(c^{l^{\prime}}\right)$ of a regular value, the global map $c^{l^{\prime}}$ is not necessarily $C^{\infty}$ locally trivial fibration (see e.g. Example 3.4.4).

\subsection{Examples}

Next we exemplify some typical anomalies of the map $c$.

Example 3.4.1 Fix a topological type of singularities (e.g. a resolution graph) and consider different analytic structures realizing it. Then not only the dimension of the target of $c: \mathrm{ECa}^{l^{\prime}}(Z) \rightarrow \mathrm{Pic}^{l^{\prime}}(Z)$ (that is, $h^{1}\left(\mathcal{O}_{Z}\right)$ ) but also the dimension of the image of $c^{l^{\prime}}$ might depend on the analytic structure of $(X, o)$. Indeed, let us fix the following graph (picture from the left):
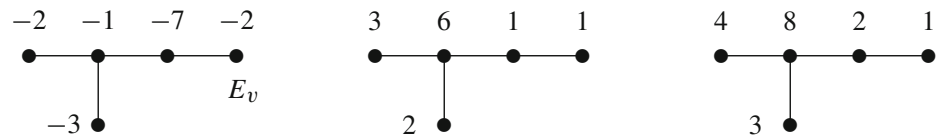

Then $(X, o)$ is a numerically Gorenstein elliptic singularity with $1 \leq p_{g} \leq 2$; for details regarding elliptic singularities see $[33,34]$. Set $-l^{\prime}:=Z_{\min }$ (the minimal cycle, which equals $E_{v}^{*}$, the cycle shown in the middle diagram), and $Z=Z_{K}$ (the last diagram), hence $\left(Z, l^{\prime}\right)=1$. Then $\mathrm{ECa}^{l^{\prime}}(Z)=\mathbb{C}$, and $\mathrm{Pic}^{l^{\prime}}(Z)=\mathbb{C}^{p_{g}}$. Write $\mathcal{L}=\mathcal{O}_{Z_{K}}\left(-Z_{\text {min }}\right)$.

If $p_{g}=2$ (hence $(X, o)$ is Gorenstein) then $\mathcal{L}$ has no fixed components [33, 5.4], and $h^{1}(Z, \mathcal{L})=1[33,2.20(\mathrm{~d})]$. Hence $\mathcal{L} \in \operatorname{im}(c)$ and $\operatorname{dim} c^{-1}(\mathcal{L})=0$ (use (3.1.8)). Therefore, $\operatorname{dim} \operatorname{im}(c)=1$.

On the other hand, if $p_{g}=1$, then $Z_{\max }>Z_{\text {min }}$, see e.g. [33, 2.20(f)]. Hence $\mathcal{L}$ has fixed components and $\mathcal{L} \notin \operatorname{im}(c)$. Since the fibers of $c$ are connected (cf. 3.1.7), $c: \mathbb{C} \rightarrow \mathbb{C}$ (with $\mathcal{L} \notin \operatorname{im}(c)$ ) cannot be quasi-finite, hence $c$ is constant and 
$\operatorname{dim} \operatorname{im}(c)=0$. (This last statement can be deduced from Theorem 4.1.1 too, or from $6.3(i) \Leftrightarrow(v)$, where we characterize completely the cases $\operatorname{dim}\left(\operatorname{im}\left(c^{l^{\prime}}\right)\right)=0$.)

Example 3.4.2 The image of $c$ usually is not closed. We construct such an example in two steps. First, assume that $(X, o)$ is a singularity with topological type given by the graph $\Gamma_{1}$ from the left
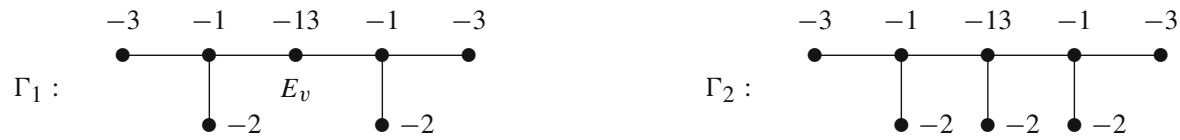

Furthermore, assume that the minimal cycle $Z_{\min }$ equals the maximal ideal cycle $Z_{\text {max }}$. In particular, $\mathcal{O}\left(-Z_{\text {min }}\right)$ has no fixed components. For a detailed study of this singularity (and any analytic type with the above graph) see [40]. Set $-l^{\prime}=Z=$ $Z_{\text {min }}=E_{v}^{*}$, and $\mathcal{L}:=\mathcal{O}_{Z}(-Z)$. Since $\left(E_{v}^{*}, E_{v}^{*}\right)=-1$ (hence $\left.\operatorname{dim} \mathrm{ECa}^{l^{\prime}}(Z)=1\right)$, and the $Z(\mathbf{t})$-coefficient $z\left(E_{v}^{*}\right)=0$ (hence $\chi\left(\mathrm{ECa}^{l^{\prime}}(Z)\right)=0$ ), one has $\mathrm{ECa}^{l^{\prime}}(Z)=$ $\mathbb{C}^{*}$. In fact, $\mathrm{ECa}^{l^{\prime}}(Z)$ is the space of divisors corresponding to the points of $E_{v}^{\text {reg }}:=$ $E_{v} \backslash \operatorname{Sing}(E) \simeq \mathbb{C}^{*}$. Using (3.1.8) and [40, §4] (which shows $h^{1}(\mathcal{L})=1$ ) one obtains that $\operatorname{dim} c^{-1}(\mathcal{L})=0$. Furthermore, $\operatorname{Pic}^{l^{\prime}}(Z)=\mathbb{C}^{2}$ (cf. 2.4), hence we get an injection $c: \mathbb{C}^{*} \hookrightarrow \mathbb{C}^{2}$. For any $q \in E_{v}^{r e g}=\mathbb{C}^{*}$ we write $\mathcal{L}_{q}:=c^{l^{\prime}}(q) \in \operatorname{Pic}^{l^{\prime}}(Z)$.

In fact, $\operatorname{im}\left(c^{l^{\prime}}\right)$ can be determined explicitly. Let $\Gamma_{l}$ and $\Gamma_{r}$ be the subgraphs consisting of the left/right cusp together with $v$. They determine minimally elliptic singularities with $p_{g}=1$, and the corresponding restrictions provide the two coordinates in $\mathrm{Pic}^{l^{\prime}}(Z)$. Applying [17, 6.11.4] for these two coordinates we get that $\operatorname{im}\left(c^{l^{\prime}}\right)$ in some affine coordinates $\left(z_{1}, z_{2}\right)$ has the form $z_{1} z_{2}=1$.

Furthermore, this situation can be used to analyze another singularity $\left(X^{\prime}, o\right)$, whose $\operatorname{im}\left(c^{\prime}\right)$ equals $\operatorname{im}(c) \backslash\{1$ point $\}$. Fix an arbitrary point $p \in E_{v}^{r e g}$, and glue to the resolution of $(X, o)$ (associated with $\left.\Gamma_{1}\right)$ another irreducible $(-2)$-exceptional curve $E_{p}^{\prime}$ transversally to $E_{v}$ at $p$. In this way we create the resolution of a new singularity ( $X^{\prime}, o$ ) with exceptional curve $E^{\prime}=\left\{E_{v}^{\prime}\right\}_{v} \cup\left\{E_{p}^{\prime}\right\}$ (with natural notations). The new graph is on the right hand side above.

In the new situation we take $-l^{\prime}=E_{v}^{\prime *}$ and $Z^{\prime}:=Z_{\text {min }}^{\prime}=E_{p}^{*}$. Then $\mathrm{ECa}^{l^{\prime}}\left(Z^{\prime}\right)$ can be identified with $\left(E_{v}^{\prime}\right)^{r e g}=E_{v}^{r e g} \backslash\{p\}=\mathbb{C}^{*} \backslash\{p\}$, and $c^{\prime}: \mathbb{C}^{*} \backslash\{p\} \rightarrow \mathbb{C}^{2}$ with the restriction of $c$ to $\mathbb{C}^{*} \backslash\{p\}$. (More precisely, for $q \in \mathbb{C}^{*} \backslash\{p\}$ one has $\left.c^{\prime}(q)\right|_{Z}=$ $\mathcal{L}_{q} \otimes \mathcal{O}_{Z}(p)$.) Since $c$ is injective, the image of $c^{\prime}$ cannot be closed. Via similar construction we can eliminate from the image of $c$ any point.

Example 3.4.3 The map $c$ usually is not a locally trivial fibration over its image, in fact, the fibers of $c$ usually are not even equidimensional.

Consider the graph $\Gamma_{1}$ from Example 3.4.2. It can be realized also by a complete intersection (splice quotient) singularity with $p_{g}=3$, cf. [40,44]. Set $-l^{\prime}=$ $2 Z_{\text {min }}=2 E_{v}^{*}$ and $Z=Z_{\text {min }}$. Then $\mathrm{ECa}^{l^{\prime}}(Z)$ is the double symmetric product of $E_{v}^{r e g}$, namely $\mathbb{C}^{*} \times \mathbb{C}^{*} / \mathbb{Z}_{2} \simeq \mathbb{C}^{*} \times \mathbb{C}$. On the other hand, Pic ${ }^{\prime}(Z)=\mathbb{C}^{2}$. (For numerical cohomological invariants see again [40].) It turns out that $c$ is dominant (use e.g. Theorem 4.1.1(3)), hence $c$ is birational, with all fibers connected. Since $Z_{\text {max }}=2 Z_{\text {min }}, \mathcal{L}=\mathcal{O}_{Z}\left(-2 Z_{\text {min }}\right)$ has no fixed components, hence $\mathcal{L} \in \operatorname{im}(c)$. Furthermore, $h^{1}(\mathcal{L})=1$ (see e.g. $[40,(5.4)]$ ), hence $\operatorname{dim} c^{-1}(\mathcal{L})=1$ by $(3.1 .8)$ 
(since $h^{1}\left(\mathcal{O}_{Z}\right)=2$ and $\left.\left(l^{\prime}, Z\right)=2\right)$. This can be seen in the following way as well. By Riemann-Roch $h^{0}(\mathcal{L})=2$ and $H^{0}\left(\mathcal{O}_{Z}\right)^{*}=\mathbb{C}^{*}$, hence by 3.1.7 $c^{-1}(\mathcal{L})$ is 1 dimensional. In particular, the fibers of $c$ are not equidimensional. (Furthermore, one can show that $\operatorname{im}(c)$ is homeomorphic to $\left(\mathbb{C}^{*}\right)^{2} \cup\{(0,0)\}$, where $(0,0)$ corresponds to $\mathcal{L}$. The map $c$ has the following description. Take the blow up $b: B \mathbb{C}^{2} \rightarrow \mathbb{C}^{2}$ of $\mathbb{C}^{2}$ at the origin. Let $L_{x}$ and $L_{y}$ be the strict transforms of $\{x=0\}$ and $\{y=0\}$. Then $\mathrm{ECa}^{l^{\prime}}(Z)$ can be identified with $B \mathbb{C}^{2} \backslash\left(L_{x} \cup L_{y}\right)$ and $c$ with the restriction of $b$ to this space.)

Example 3.4.4 Even if all the fibers have the same dimension (and by Theorem 3.2.2 they are smooth) the topology of some fibers might jump. Take for example the graph

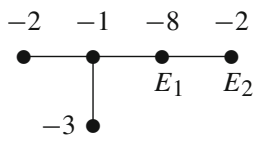

It supports a non-numerically Gorenstein elliptic singularity. Recall that if $C$ denotes the elliptic cycle (here it is supported on the union of all irreducible exceptional curves except $\left.E_{2}\right)$, and $\left(C, Z_{\text {min }}\right)<0$, then the length of the elliptic sequence is one, cf. $[58,59]$. Hence, for any analytic realization, $p_{g}=1$. Take $-l^{\prime}=Z=Z_{\min }=$ $E_{1}^{*}+E_{2}^{*}$. A computation shows that $\mathrm{ECa}^{l^{\prime}}(Z)=\mathbb{C}^{2} \backslash\{0\}$. Then $c: \mathbb{C}^{2} \backslash\{0\} \rightarrow \mathbb{C}$ can be identified with the restriction to $\mathbb{C}^{2} \backslash\{0\}$ of the linear projection $\mathbb{C}^{2} \rightarrow \mathbb{C}$. Hence the generic fiber is $\mathbb{C}$ while there is a special fiber $\simeq \mathbb{C}^{*}$. By this correspondence $\operatorname{Pic}^{l^{\prime}}(Z)=\mathbb{C}$ is identified by $E_{1} \backslash E_{\text {node }}$. The generic fibers correspond to the divisors $\{p, q\}$, where $p \in E_{1}^{r e g} \simeq \mathbb{C}^{*}$, and $q \in E_{2}^{r e g} \simeq \mathbb{C}$; they are sent by $c$ to $p \in E_{1}^{r e g} \subset$ $E_{1} \backslash E_{\text {node }} \simeq \operatorname{Pic}^{l^{\prime}}(Z)$. Since $q$ can be any point on $E_{2}^{r e g}$, the fibers are $\mathbb{C}$. On the other hand, any divisor given by a smooth cut at $E_{1} \cap E_{2}$, transversal to both $E_{1}$ and $E_{2}$, (parametrized by the slope $\mathbb{C}^{*}$ ) is sent by $c$ to $E_{1} \cap E_{2}$, whose fiber is exactly this parameter space $\mathbb{C}^{*}$.

Example 3.4.5 For an example when the image of $c$ is singular see Example 12.9.1.

\subsection{The topology of the fibers of $c$ and the coefficients of the Poincaré series}

Let us analyse again the fibers of $c: \mathrm{ECa}^{l^{\prime}}(Z) \rightarrow \operatorname{Pic}^{l^{\prime}}(Z), Z \geq E$. Assume that $\mathcal{L} \in \operatorname{im}(c)$. Then $\left\{H_{v}:=H^{0}\left(Z-E_{v}, \mathcal{L}\left(-E_{v}\right)\right)\right\}_{v \in \mathcal{V}}$ is a proper linear subspace arrangement in $H^{0}:=H^{0}(Z, \mathcal{L})$. For any subset $\emptyset \neq I \subset \mathcal{V}$ write $H_{I}:=\cap_{v \in I} H_{v}$, and introduce also $H_{\emptyset}:=H^{0}$. Note that the topological Euler characteristic satisfies $\chi_{\text {top }}\left(\mathbb{P} H_{I}\right)=\operatorname{dim} H_{I}$, hence by the inclusion-exclusion principle one obtains

$$
\begin{aligned}
\chi_{\text {top }}\left(\mathbb{P}\left(H^{0} \backslash \cup_{v} H_{v}\right)\right) & =\sum_{I \subset \mathcal{V}}(-1)^{|I|} \operatorname{dim} H_{I} \\
& =\sum_{I}(-1)^{|I|+1} \operatorname{codim}\left(H_{I} \subset H^{0}\right) .
\end{aligned}
$$


In particular the analytic invariant $p_{Z, \mathcal{L}}$ (cf. 2.3.6) equals the topological Euler characteristic of the corresponding linear subspace arrangement complement, $p_{Z, \mathcal{L}}=$ $\chi_{\text {top }}\left(\mathbb{P}\left(H^{0}(Z, \mathcal{L})_{\text {reg }}\right)\right)$. Using Lemmas 3.1.4 and 3.1.7 this reads as

$$
p_{Z, \mathcal{L}}=\chi_{\text {top }}\left(c^{-1}(\mathcal{L})\right)
$$

This fact links the coefficients of the topological series $Z(\mathbf{t})$ and the numerical analytical invariants $p_{Z, \mathcal{L}}$ : the Euler characteristic of the total space $\mathrm{ECa}^{l^{\prime}}$ is $z\left(-l^{\prime}\right)$, while the Euler characteristic of each fiber $c^{-1}(\mathcal{L})(\mathcal{L} \in \operatorname{im}(c))$ is $p_{Z, \mathcal{L}}$.

Example 3.5.2 Assume that $(X, o)$ is rational. Then $\operatorname{Pic}^{l^{\prime}}(Z)$ is a point: if $c_{1}(\mathcal{L})=l^{\prime}$ then $\mathcal{L}=\mathcal{O}\left(l^{\prime}\right)$. Hence $\mathrm{ECa}^{l^{\prime}}$ is the unique fiber $c^{-1}\left(\mathcal{O}\left(l^{\prime}\right)\right)$. Therefore, $z\left(-l^{\prime}\right)=$ $p_{Z, \mathcal{O}\left(l^{\prime}\right)}\left(l^{\prime} \in-\mathcal{S}^{\prime}\right)$, or $Z(\mathbf{t})=P_{Z, \mathcal{O}}(\mathbf{t})$. This generalizes the similar identity proved in $[7,8,36,37]$ valid for $Z \gg 0$ (or, for $\widetilde{X}$ ).

This identity $Z(\mathbf{t})=P_{\mathcal{O}_{\tilde{X}}}(\mathbf{t})$ is valid for a more general family of singularities, namely for splice quotient singularities [36,37]. (This family was introduced by Neumann and Wahl in $[45,46])$. This identity reinterpreted in our present language says that for any $-l^{\prime} \in \mathcal{S}^{\prime}$ and $Z \gg 0$ the Euler characteristic of the total space $\mathrm{ECa}^{l^{\prime}}(Z)$ and the Euler characteristic of the very special fiber $c^{-1}\left(\mathcal{O}\left(l^{\prime}\right)\right)$ (over the unique natural line bundle) coincide.

Conjecture 3.5.3 For a splice quotient singularity and $-l^{\prime} \in \mathcal{S}^{\prime}$ the fiber $c^{-1}\left(\mathcal{O}\left(l^{\prime}\right)\right)$ is a topological deformation retract of $\mathrm{ECa}^{l^{\prime}}(Z)$.

A detailed study of the Abel map in the case of splice quotient singularities will appear in one of the parts of the present series of articles.

In the present work we wish to focus (instead/besides of the ' $P_{\mathcal{O}}=Z$ identity') on the more complex package of invariants provided by (all the fibers of) $c$. In particular, we analyse other, less specific fibers as well, e.g. the generic fibers over $\operatorname{im}(c)$.

\section{Characterization of $c^{c^{\prime}}$ dominant}

4.1. In order to determine properties of line bundles $\mathcal{L} \in \operatorname{Pic}(Z)$ with given Chern class we need first to understand the situations when $c^{l^{\prime}}$ is dominant.

Theorem 4.1.1 Fix $l^{\prime} \in-\mathcal{S}^{\prime}, Z \geq E$ as above, and consider $c^{l^{\prime}}: \mathrm{ECa}^{l^{\prime}}(Z) \rightarrow$ $\operatorname{Pic}^{l^{\prime}}(Z)$.

(1) $c^{l^{\prime}}$ is dominant if and only if $H^{0}(Z, \mathcal{L})_{\mathrm{reg}} \neq \emptyset$ for generic $\mathcal{L} \in \operatorname{Pic}^{l^{\prime}}(Z)$.

(2) If $c^{l^{\prime}}$ is dominant then $h^{1}(Z, \mathcal{L})=0$ for generic $\mathcal{L} \in \operatorname{Pic}^{l^{\prime}}(Z)$.

(3) $c^{l^{\prime}}$ is dominant if and only if $\chi\left(-l^{\prime}\right)<\chi\left(-l^{\prime}+l\right)$ for all $0<l \leq Z, l \in L$. In particular, the fact that $c^{l^{\prime}}$ is dominant is independent of the analytic structure supported by $\Gamma$ and it can be characterized topologically (and explicitly).

Proof For (1) use Lemma 3.1.4. For (2) note that for $c$ dominant the dimension of $\mathrm{ECa}^{l^{\prime}}(Z)$ is the sum of the dimensions of the generic fiber and of the base (which 
equals $\left.h^{1}\left(\mathcal{O}_{Z}\right)\right)$. Hence, by (3.1.8) and 3.1.10 $(1), h^{0}(Z, \mathcal{L})=\operatorname{dim} c^{-1}(\mathcal{L})+h^{0}(Z)=$ $\left(l^{\prime}, Z\right)-h^{1}(Z)+h^{0}(Z)=\left(l^{\prime}, Z\right)+\chi(Z)=\chi(Z, \mathcal{L})$.

(3) First note that for any cycle $l \in L, 0<l \leq Z$, and any $\mathcal{L} \in \operatorname{Pic}^{\prime}(Z)$, one has

$$
\chi\left(-l^{\prime}\right) \geq \chi\left(-l^{\prime}+l\right) \Leftrightarrow \chi(Z, \mathcal{L}) \leq \chi(Z-l, \mathcal{L}(-l)),
$$

where, by convention, $\chi(Z-l, \mathcal{L}(-l))$ is zero whenever $l=Z$.

Assume that $c$ is dominant and the equivalent conditions from (4.1.2) are satisfied for some $l$, where $0<l \leq Z$. Take a generic $\mathcal{L} \in \operatorname{Pic}^{l^{\prime}}(Z)$. Hence $H^{0}(Z, \mathcal{L})_{\text {reg }} \neq \varnothing($ cf. part $(1))$ and $\chi(Z, \mathcal{L})=h^{0}(Z, \mathcal{L})$ by part $(2)$. Hence $h^{0}(Z, \mathcal{L})=\chi(Z, \mathcal{L}) \leq \chi(Z-$ $l, \mathcal{L}(-l)) \leq h^{0}(Z-l, \mathcal{L}(-l))$. Therefore, by the cohomological exact sequence of $\left.0 \rightarrow \mathcal{L}(-l)\right|_{Z-l} \rightarrow \mathcal{L}$, we necessarily have equality $H^{0}(Z-l, \mathcal{L}(-l))=H^{0}(Z, \mathcal{L})$. Then for any $E_{v}$ in the support of $l$ we also have equality $H^{0}\left(Z-E_{v}, \mathcal{L}\left(-E_{v}\right)\right)=$ $H^{0}(Z, \mathcal{L})$, hence $H^{0}(Z, \mathcal{L})_{\text {reg }}=\emptyset$, which leads to a contradiction.

Assume that $\chi\left(-l^{\prime}\right)<\chi\left(-l^{\prime}+l\right)$ for any $0<l \leq Z$. This, for $l=Z$, implies $\chi(Z, \mathcal{L})>0$, hence necessarily $h^{0}(Z, \mathcal{L})>0$ for any $\mathcal{L} \in \operatorname{Pic}^{l^{\prime}}(Z)$. If for a generic $\mathcal{L}$ one has $H^{0}(Z, \mathcal{L})_{\text {reg }}=\emptyset$, then there exists $E_{v}$ such that $H^{0}(Z, \mathcal{L})=H^{0}(Z-$ $\left.E_{v}, \mathcal{L}\left(-E_{v}\right)\right)$. If $H^{0}\left(Z-E_{v}, \mathcal{L}\left(-E_{v}\right)\right)_{\text {reg }}=\emptyset$ again, then we continue the procedure. In this way we obtain a cycle $0<l \leq Z$ such that $H^{0}(Z-l, \mathcal{L}(-l))=H^{0}(Z, \mathcal{L})$ and $H^{0}(Z-l, \mathcal{L}(-l))_{\text {reg }} \neq \emptyset$. Note that for $\mathcal{L}$ generic $\left.\mathcal{L}(-l)\right|_{Z-l} \in \operatorname{Pic}^{l^{\prime}-l}(Z-l)$ is generic as well. Hence $c^{l^{\prime}-l}$ is dominant and by (1)-(2) $h^{1}(Z-l, \mathcal{L}(-l))=0$. Therefore, $\chi(Z, \mathcal{L}) \leq h^{0}(Z, \mathcal{L})=h^{0}(Z-l, \mathcal{L}(-l))=\chi(Z-l, \mathcal{L}(-l))$, which by (4.1.2) reads as $\chi\left(-l^{\prime}\right) \geq \chi\left(-l^{\prime}+l\right)$, a contradiction.

Example 4.1.3 The statement of Theorem 4.1.1(3) is non-trivial even for $l^{\prime}=0$. In this case, since $\mathrm{ECa}^{0}$ is a point, $c^{0}$ is dominant if and only if $\operatorname{Pic}^{0}(Z)$ is a point, that is, $h^{1}\left(\mathcal{O}_{Z}\right)=0$. Hence part (3) reads as the following topological characterization of the vanishing of $h^{1}\left(\mathcal{O}_{Z}\right)$ : For any normal surface singularity and any cycle $Z>0$, $h^{1}\left(\mathcal{O}_{Z}\right)=0$ if and only if $\chi(l)>0$ for any $0<l \leq Z$. (This is a generalization of the rationality criterion of Artin [2,3], which corresponds to $Z \gg 0$.)

Remark 4.1.4 Above, we assumed that $Z \geq E$. This is not really necessary: if the support $|Z|$ of $Z$ is smaller then one can restrict all the objects to $|Z|$, and the above statements (and also the next Theorem 5.3.1) remain valid. (Along the restriction, $\widetilde{X}$ will be replaced by a small convenient neighbourhood of $\cup_{E_{v} \subset|Z|} E_{v}$, and $L$ by $\mathbb{Z}\left\langle E_{v}\right\rangle_{\left.E_{v} \subset|Z| \cdot\right)}$

4.1.5 The semigroup of dominant Chern classes $(Z \gg 0)$. Theorem 4.1.1(3) motivates the introduction of the following combinatorial set

$$
\mathcal{S}_{\text {dom }}^{\prime}:=\left\{-l^{\prime} \mid \chi\left(-l^{\prime}\right)<\chi\left(-l^{\prime}+l\right) \text { for all } l \in L_{>0}\right\} .
$$

By definition, $-l^{\prime} \in \mathcal{S}_{d o m}^{\prime}$ if and only if $c^{l^{\prime}}$ is dominant for $Z \gg 0$.

Sometimes it is more convenient to use the next equivalent form (note the sign modification):

$$
\mathcal{S}_{\text {dom }}^{\prime}=\left\{l^{\prime} \mid \chi(l)>\left(l^{\prime}, l\right) \text { for all } l \in L_{>0}\right\}
$$


Lemma 4.1.7 $\mathcal{S}_{\text {dom }}^{\prime}$ has the following properties:

(i) $\mathcal{S}_{\text {dom }}^{\prime} \subset \mathcal{S}^{\prime}$.

(ii) $0 \in \mathcal{S}_{\text {dom }}^{\prime}$ iff $L$ is rational. More generally, for $I \subset \mathcal{V}$ and $n_{v}>0$ for all $v \in I$, if $\sum_{v \in I} n_{v} E_{v}^{*} \in \mathcal{S}_{d o m}^{\prime}$ then the components of $\cup_{v \notin I} E_{v}$ are rational. Hence, in general, $\mathcal{S}^{\prime} \backslash \mathcal{S}_{\text {dom }}^{\prime}$ is infinite.

(iii) $\mathcal{S}^{\prime} \cap\left(Z_{K} / 2+\mathcal{S}_{\mathbb{Q}}^{\prime}\right) \subset \mathcal{S}_{\text {dom }}^{\prime}$, where $\mathcal{S}_{\mathbb{Q}}^{\prime}:=\left\{l^{\prime} \in L \otimes \mathbb{Q}:\left(l^{\prime}, E_{v}\right) \leq 0\right.$ for all $\left.v\right\}$.

(iv) $\mathcal{S}_{\text {dom }}^{\prime}$ is a semigroup (not necessarily with identity element).

(v) $\mathcal{S}_{d o m}^{\prime}$ is an $\mathcal{S}^{\prime}$-module, that is, if $l_{1}^{\prime} \in \mathcal{S}_{\text {dom }}^{\prime}, l_{2}^{\prime} \in \mathcal{S}^{\prime}$ then $l_{1}^{\prime}+l_{2}^{\prime} \in \mathcal{S}_{\text {dom }}^{\prime}$.

(vi) $\mathcal{S}_{d o m}^{\prime}$ is min-stable, like $\mathcal{S}^{\prime}$, that is, if $l_{1}^{\prime}, l_{2}^{\prime} \in \mathcal{S}_{\text {dom }}^{\prime}$ then $m:=\min \left\{l_{1}^{\prime}, l_{2}^{\prime}\right\} \in \mathcal{S}_{\text {dom }}^{\prime}$.

Proof For $(i)$ use (3.1.5) or (4.1.6). ( $i$ i ) follow from Artin's criterion. ( $i i i)$ is clear. For $(i v)-(v)$ use (4.1.6): if $\chi(l)>\left(l_{1}^{\prime}, l\right)$ and $0>\left(l_{2}^{\prime}, l\right)($ cf. $(i))$, then $\chi(l)>\left(l_{1}^{\prime}+l_{2}^{\prime}, l\right)$. Next we prove $(v i)$.

We wish to show that $\chi(l)>(m, l)$ for any $l>0$. Set $x_{i}=l_{i}^{\prime}-m(i=1,2)$. Assume first that $l \geq x_{1}$, and write $l=x_{1}+z$. Then from the assumptions $\chi\left(x_{1}\right) \geq\left(m+x_{2}, x_{1}\right)$ (equality only if $\left.x_{1}=0\right)$ and $\chi(z) \geq\left(m+x_{1}, z\right)$ (equality only if $z=0$ ). These added provide $\chi(l)>(m, l)+\left(x_{1}, x_{2}\right) \geq(m, l)$.

Next assume that $l \ngtr x_{1}$, and choose $u_{1}>0$ minimal, supported by the support of $x_{1}$, such that $l+u_{1} \geq x_{1}$. Then the hypothesis applied for $l_{1}^{\prime}=m+x_{1}$ gives $\chi\left(l+u_{1}-x_{1}\right) \geq\left(m+x_{1}, l+u_{1}-x_{1}\right)$ (equality only if $\left.l+u_{1}-x_{1}=0\right)$ and applied for $l_{2}^{\prime}=m+x_{2}$ gives $\chi\left(x_{1}-u_{1}\right) \geq\left(m+x_{2}, x_{1}-u_{1}\right)$ (equality only if $x_{1}-u_{1}=0$ ). These added gives $\chi(l)-(m, l)>\left(u_{1}, l+u_{1}-x_{1}\right)+\left(x_{2}, x_{1}-u_{1}\right) \geq 0$.

Corollary 4.1.8 (i) For any $-l^{\prime} \in L^{\prime}$ there exists a unique minimal $l_{d o m} \in L_{\geq 0}$ with $-l^{\prime}+l_{\text {dom }} \in \mathcal{S}_{\text {dom }}^{\prime}$

(ii) $l_{d o m}$ can be found by the following algorithm (see the analogy with [25]). We construct a computation sequence $\left\{z_{i}\right\}_{i=0}^{t}$, (where $z_{i+1}=z_{i}+E_{v(i)}$ for some $v(i) \in \mathcal{V})$ as follows. Fix a generic line bundle $\mathcal{L} \in \operatorname{Pic}^{l^{\prime}}(\widetilde{X})$. Start with $z_{0}=0$. Assume that $z_{i}$ is already constructed and consider $\mathcal{L}\left(-z_{i}\right)$. If $H^{0}\left(\mathcal{L}\left(-z_{i}\right)\right)$ has no fixed components then stop and $z_{i}$ is the last term $z_{t}$. If $H^{0}\left(\mathcal{L}\left(-z_{i}\right)\right)$ has a fixed component, choose one of them, say $E_{v(i)}$, and write $z_{i+}:=z_{i}+E_{v(i)}$ and repeat the algorithm. Then this procedure stops after finitely many steps and $z_{t}=l_{d o m}$.

Proof $(i)$ Set $\mathcal{D}:=\left(-l^{\prime}+L_{\geq 0}\right) \cap \mathcal{S}_{d o m}^{\prime}$. Then $\mathcal{D} \neq \emptyset$ by $4.1 .7($ iii $)$ and it has a unique minimal element by 4.1.7(vi).

(ii) We show inductively that $z_{i} \leq l_{d o m}$ and the construction stops exactly when $z_{i}=l_{d o m}$. Note that $z_{0}=0 \leq l_{d o m}$. If $z_{i}=l_{d o m}$ then $-l^{\prime}+z_{i} \in \mathcal{S}_{d o m}^{\prime}$, hence by Theorem 4.1.1(1) $H^{0}\left(\mathcal{L}\left(-z_{i}\right)\right)$ has no fixed components, hence we have to stop.

If, by induction $z_{i}<l_{d o m}$, we have to show that the algorithm does not stop and $z_{i+1} \leq l_{d o m}$ as well. Indeed, if $-l^{\prime}+z_{i}<-l^{\prime}+l_{d o m}$ then $-l^{\prime}+z_{i} \notin \mathcal{S}_{d o m}^{\prime}$ by the minimality of $l_{d o m}$, hence by Theorem 4.1.1 $H^{0}\left(\mathcal{L}\left(-z_{i}\right)\right)$ has fixed components. Hence the procedure continues. Note also that the generic section of $H^{0}\left(\mathcal{L}\left(-l_{\text {dom }}\right)\right)$ has no fixed components, hence the fixed components of $H^{0}\left(\mathcal{L}\left(-z_{i}\right)\right)$ should be supported on $l_{d o m}-z_{i}$. Hence $z_{i}+E_{v(i)} \leq l_{d o m}$.

Remark 4.1.9 Though $\mathcal{S}_{\text {dom }}^{\prime}$ is defined above combinatorially/topologically, it shares (see e.g. (iv) and (vi)) several properties of an analytic semigroup associated with an 
analytic structure supported on $\Gamma$. This 'coincidence' will be clarified completely in the forthcoming part [32], where we prove that the analytic semigroup associated with the generic analytic structure is exactly $\mathcal{S}_{\text {dom }}^{\prime} \cup\{0\}$.

\section{Cohomology of line bundles and $\operatorname{dim} \operatorname{im}\left(c^{\prime}\right)$}

\subsection{Line bundles with $c_{1}(\mathcal{L}) \notin-\mathcal{S}^{\prime}$}

Recall that by (3.1.5) $\mathrm{ECa}^{l^{\prime}}(Z) \neq \emptyset$ iff $l^{\prime} \in-\mathcal{S}^{\prime}$. Hence any result based on the Abel map uses $l^{\prime} \in-\mathcal{S}^{\prime}$. E.g., in this section we establish a sharp lower bound for $h^{1}(Z, \mathcal{L})$ whenever $c_{1}(\mathcal{L})=l^{\prime} \in-\mathcal{S}^{\prime}$. Before we provide that statement we wish to emphasise that this extends automatically to the case of all bundles $\mathcal{L}$, even if $c_{1}(\mathcal{L}) \notin-\mathcal{S}^{\prime}$.

Indeed, it is known that for any $x \in L^{\prime}$ there exist $s(x)=x+l \in L^{\prime}$ with the following properties: (a) $s(x) \in \mathcal{S}^{\prime}$, (b) $l \in L_{\geq 0}$, (c) $s(x)$ is minimal with properties (a)(b). Furthermore, the cycle $l$ can be determined explicitly using a generalized Laufer sequence [35, Prop. 4.3.3]. One constructs a computation sequence $\left\{z_{i}\right\}_{i=0}^{t}, z_{0}=0$, $z_{i+1}=z_{i}+E_{v(i)}$ for some $v(i) \in \mathcal{V}$ inductively as follows. If $x+z_{i} \in \mathcal{S}^{\prime}$ then one stops, and automatically $i=t$ and $z_{i}=l$. If there exists $E_{v}$ with $\left(x+z_{i}, E_{v}\right)>0$ then choose $E_{v(i)}$ as such an $E_{v}$, and one defines $z_{i+1}=z_{i}+E_{v(i)}$. Along the computation sequence $i \mapsto \chi\left(x+z_{i}\right)$ is decreasing. Furthermore, if $Z>l$, then the sequence applied for $x=-l^{\prime}=-c_{1}(\mathcal{L})$, we get that $h^{0}\left(Z-z_{i}, \mathcal{L}\left(-z_{i}\right)\right)$ is constant, and

$$
h^{1}(Z, \mathcal{L})=h^{1}(Z-l, \mathcal{L}(-l))-\chi\left(\left.\mathcal{L}\right|_{l}\right) \text { and } c_{1}(\mathcal{L}(-l)) \in-\mathcal{S}^{\prime}
$$

Here, clearly, $\chi\left(\left.\mathcal{L}\right|_{l}\right)=\left(l^{\prime}, l\right)+\chi(l)=\chi\left(-l^{\prime}+l\right)-\chi\left(-l^{\prime}\right)$. If $l \not Z Z$, then one constructs a computation sequence inductively as follows: if $-l^{\prime}+z_{i} \in \mathcal{S}^{\prime}\left(\left|Z-z_{i}\right|\right)$ (the Lipman cone associated with the support $\left.\left|Z-z_{i}\right|\right)$ then one stops, otherwise there exists $E_{v(i)}$ (identified as above) supported on $Z-z_{i}$, which provides $z_{i+1}=z_{i}+E_{v(i)}$. In particular, for any $\mathcal{L} \in \operatorname{Pic}(Z)$, there exists $l \in L_{\geq 0}$ such that $-c_{1}(\mathcal{L}(-l)) \in$ $\mathcal{S}^{\prime}(|Z-l|)$, and (5.1.1) holds.

Summarized, the computation of any $h^{1}(Z, \mathcal{L})$, up to the topology of the graph, can be reduced to the case $-c_{1}(\mathcal{L}) \in \mathcal{S}^{\prime}$ (maybe supported on a smaller set).

\subsection{Semicontinuity}

We emphasise another specific fact as well: since $c^{l^{\prime}}$ is not proper, the semicontinuity of the dimension of the fiber (with respect to the points of the target) does not follow automatically from the general theory. Nevertheless, we have the following result.

Lemma 5.2.1 $h^{0}(Z, \mathcal{L})$ and $h^{1}(Z, \mathcal{L})$ are semicontinuous with respect to $\mathcal{L} \in \operatorname{Pic}^{l^{\prime}}(Z)$. In particular, via (3.1.8), $\operatorname{dim} c^{-1}(\mathcal{L})$ is also semicontinuous with respect to $\mathcal{L} \in$ $\operatorname{Pic}^{l^{\prime}}(Z)$.

Proof Consider a covering by small balls $\left\{U_{\alpha}\right\}_{\alpha}$ of $\widetilde{X}$. Since $\left.\mathcal{L}\right|_{U_{\alpha}}$ is trivial for any $\alpha$ and $\mathcal{L}, H^{0}(Z, \mathcal{L})=\operatorname{ker}\left(\delta_{\mathcal{L}}: \oplus_{\alpha} H^{0}\left(\left.\mathcal{O}_{Z}\right|_{U_{\alpha}}\right) \rightarrow \oplus_{\alpha \neq \beta} H^{0}\left(\left.\mathcal{O}_{Z}\right|_{U_{\alpha} \cap U_{\beta}}\right)\right)$, where 
the $\mathcal{L}$-dependence is codified in $\delta_{\mathcal{L}}$. But the corank of the linear map (hence, consequently $h^{0}(Z, \mathcal{L})$ too $)$ is semicontinuous. The semicontinuity of $h^{1}(Z, \mathcal{L})$ follows by Riemann-Roch.

5.3. We prove the following sharp semicontinuity inequality.

Theorem 5.3.1 (1) Fix an arbitrary $l^{\prime} \in L^{\prime}$. Then for any $\mathcal{L} \in \operatorname{Pic}^{l^{\prime}}(Z)$ one has

$$
\begin{aligned}
h^{1}(Z, \mathcal{L}) & \geq \chi\left(-l^{\prime}\right)-\min _{0 \leq l \leq Z, l \in L} \chi\left(-l^{\prime}+l\right), \text { or, equivalently } \\
h^{0}(Z, \mathcal{L}) & \geq \max _{0 \leq l \leq Z, l \in L} \chi(Z-l, \mathcal{L}(-l)) \\
& =\max _{0 \leq l \leq Z, l \in L}\left\{\chi(Z-l)+\left(Z-l, l^{\prime}-l\right)\right\} .
\end{aligned}
$$

Furthermore, if $\mathcal{L}$ is generic in $\mathrm{Pic}^{l^{\prime}}(Z)$ then in both inequalities we have equality. In particular, $h^{*}(Z, \mathcal{L})$ is topological and explicitly computable from $L$, whenever $\mathcal{L}$ is generic.

(2) Assume that $l^{\prime} \in-\mathcal{S}^{\prime}$ and $c^{l^{\prime}}$ is not dominant. Then the inequalities in (5.3.2) are strict for any $\mathcal{L} \in \operatorname{im}\left(c^{l^{\prime}}\right)$.

Proof (1) The two inequalities (and the corresponding equalities) are equivalent by Riemann-Roch. We will prove the statement for $h^{0}$. For any $l$ and $\mathcal{L}$ (by a cohomological exact sequence) one has

$$
h^{0}(Z, \mathcal{L}) \geq h^{0}(Z-l, \mathcal{L}(-l)) \geq \chi(Z-l, \mathcal{L}(-l))
$$

hence the inequality follows. We need to show the opposite inequality for $\mathcal{L}$ generic. Clearly, if $h^{0}(Z, \mathcal{L})=0$, then the opposite inequality follows (take e.g. $l=Z$ ). Hence, assume $h^{0}(Z, \mathcal{L}) \neq 0$. Then, as in the proof of Theorem 4.1.1, there exists $0 \leq l<Z$ such that $h^{0}(Z, \mathcal{L})=h^{0}(Z-l, \mathcal{L}(-l))$ and $H^{0}(Z-l, \mathcal{L}(-l))_{\text {reg }} \neq \emptyset$. In this case $l^{\prime}-l \in-\mathcal{S}^{\prime}$ by (3.1.5) and (by Theorem 4.1.1) $h^{1}(Z-l, \mathcal{L}(-l))=0$ as well. Hence $h^{0}(Z, \mathcal{L})=\chi(Z-l, \mathcal{L}(-l)) \leq \max _{0 \leq l \leq Z} \chi(Z-l, \mathcal{L}(-l))$.

(2) Assume that $h^{0}(Z, \mathcal{L})=\max _{0 \leq l \leq Z} \chi(Z-l, \mathcal{L}(-l))$. If the max at the right hand side can be realized by a certain $l_{0}>0$ then using (5.3.3) for $l_{0}$ we get that $h^{0}(Z, \mathcal{L})=h^{0}\left(Z-l_{0}, \mathcal{L}\left(-l_{0}\right)\right)$, hence $\mathcal{L}$ has fixed components, that is, $\mathcal{L} \notin \operatorname{im}\left(c^{l^{\prime}}\right)$. On the other hand, if the max is realized only by $l=0$, then $c^{l^{\prime}}$ is dominant by Theorem 4.1.1(3).

Since $H^{1}(\widetilde{X}, \mathcal{L})=\lim _{\leftarrow, Z} H^{1}(Z, \mathcal{L})$, cf. [17, Th. 11.1], we obtain the following.

Corollary 5.3.4 For $l^{\prime} \in L^{\prime}$ and any $\mathcal{L} \in \operatorname{Pic}^{l^{\prime}}(\tilde{X})$ one has $h^{1}(\tilde{X}, \mathcal{L}) \geq \chi\left(-l^{\prime}\right)-$ $\min _{l \in L \geq 0} \chi\left(-l^{\prime}+l\right)$. Equality holds whenever $\mathcal{L}$ is generic in $\operatorname{Pic}^{l^{\prime}}(\widetilde{X})$. Furthermore, if $l^{\prime} \in-\mathcal{S}^{\prime}$ and $c^{l^{\prime}}$ is not dominant, then $h^{1}(\tilde{X}, \mathcal{L})>\chi\left(-l^{\prime}\right)-\min _{l \in L_{\geq 0}} \chi\left(-l^{\prime}+l\right)$ whenever $\mathcal{L} \in \operatorname{im}\left(c^{l^{\prime}}\right)$. 
Example 5.3.5 Assume that $l^{\prime}=0$ and $h^{1}\left(\mathcal{O}_{Z}\right) \neq 0$. Then $c^{0}$ is not dominant, hence $h^{1}(Z, \mathcal{L}) \geq-\min _{0 \leq l \leq Z} \chi(l)$ for any $\mathcal{L}$, and $h^{1}\left(\mathcal{O}_{Z}\right) \geq 1-\min _{0 \leq l \leq Z} \chi(l)$.

Moreover, for generic $\mathcal{L} \in \operatorname{Pic}^{0}(Z)$ one has $h^{1}(Z, \mathcal{L})=-\min _{0 \leq l \leq Z} \chi(l)$. This for $Z \gg 0$ and $\Gamma$ elliptic reads as $h^{1}(\widetilde{X}, \mathcal{L})=0$; this fact for minimally elliptic $\Gamma$ was proved by Laufer in [26], and for arbitrary elliptic case in [33].

Example 5.3.6 Consider the situation of Corollary 5.3.4. For certain topological types one can find for any $l^{\prime}$ explicitly a cycle $l_{\text {min }} \in L_{\geq 0}$ which realizes $\min _{l \in L_{>0}} \chi\left(-l^{\prime}+\right.$ $l)=\chi\left(-l^{\prime}+l_{\text {min }}\right)$. Indeed, consider the construction $x \mapsto x+l=s(x)$ described in 5.1. Since $\chi$ is decreasing along the sequence, $(*) \chi(s(x)) \leq \chi(x)$. Next, assume e.g. that the lattice has the property that $\chi(l) \geq 0$ for all $l \in L_{\geq 0}$ (hence the graph is either rational or elliptic). Then for any $s \in \mathcal{S}^{\prime}$ one has $(* *) \chi(s) \leq \chi(s+l)$ for all $l \in L_{\geq 0}$.

We claim that for rational and elliptic singularities $\min _{l \in L_{\geq 0}} \chi\left(-l^{\prime}+l\right)=$ $\chi\left(s\left(-l^{\prime}\right)\right)$.

Indeed, by $(*)$ one has $\chi\left(-l^{\prime}+l_{\text {min }}\right) \geq \chi\left(s\left(-l^{\prime}+l_{\text {min }}\right)\right)$, and by the universal property of the operator $s$ one also has $s\left(-l^{\prime}+l_{\text {min }}\right) \geq s\left(-l^{\prime}\right)$, hence by $(* *) \chi\left(s\left(-l^{\prime}+\right.\right.$ $\left.\left.l_{\text {min }}\right)\right) \geq \chi\left(s\left(-l^{\prime}\right)\right)$.

In particular, for rational and elliptic germs $h^{1}(\tilde{X}, \mathcal{L})=\chi\left(-l^{\prime}\right)-\chi\left(s\left(-l^{\prime}\right)\right)$ whenever $\mathcal{L}$ is generic.

See also Corollary 5.5.2, where we prove for any $(X, o)$ the existence of a unique minimal cycle with the property of $l_{\min }$.

5.4. In parallel to $\mathcal{S}_{d o m}^{\prime}$ (see 4.1.5), Corollary 5.3.4 indicates another subset of $L^{\prime}$ :

$$
V a n^{\prime}:=\left\{-l^{\prime} \mid \chi\left(-l^{\prime}\right) \leq \chi\left(-l^{\prime}+l\right) \text { for all } l \in L_{\geq 0}\right\} .
$$

This indexes those cycles $-l^{\prime}$ for which $h^{1}(\tilde{X}, \mathcal{L})=0$ for generic $\mathcal{L} \in \operatorname{Pic}^{l^{\prime}}(\tilde{X})$.

For arbitrary line bundles $\mathcal{L} \in \operatorname{Pic}^{l^{\prime}}(\widetilde{X})$ the existent vanishing theorems formulate sufficient (but usually not necessary) criterions. E.g., $h^{1}(\widetilde{X}, \mathcal{L})=0$ for any $(X, o)$ whenever $-l^{\prime} \in Z_{K}+\mathcal{S}^{\prime}$ (this is the so-called Grauert-Riemenschneider vanishing) $[15,25,56]$, or, for rational $(X, o)$ whenever $-l^{\prime} \in \mathcal{S}^{\prime}$ (Lipman's Criterion) [27]. Even so, Corollary 5.3.4 provides a necessary and sufficient vanishing condition for generic line bundles, which, surprisingly, is independent of the analytic structure of $(X, o)$. $V_{a n^{\prime}}$ lists precisely the corresponding Chern classes.

For rational singularities (since $h^{1}(\widetilde{X}, \mathcal{L})$ depends only on $c_{1}(\mathcal{L})$, cf. [35, 4.3.3]), $h^{1}(\tilde{X}, \mathcal{L})=0$ for any line bundle with fixed $c_{1}(\mathcal{L})$ exactly when $-c_{1}(\mathcal{L}) \in V a n^{\prime}$. This is not valid for more general singularities: $-l^{\prime} \in V a n^{\prime}$ does not guarantee the vanishing $h^{1}(\widetilde{X}, \mathcal{L})=0$ for non-generic (hence for arbitrary) bundles. E.g., in the elliptic case, $0 \in \operatorname{Van}^{\prime}$, however $h^{1}\left(\widetilde{X}, \mathcal{O}_{\widetilde{X}}\right)=p_{g}>0$.

Though most of the statements of the next lemma will not be needed in this first part of the series of articles, for completeness and further references we list some properties of $\operatorname{Van}^{\prime}$ (which can be compared e.g. with those from Lemma 4.1.7). Note that a semigroup module structure of type (iv) usually is not studied/observed in vanishing theorems.

Lemma 5.4.2 Van' satisfies the following properties: 
(i) $\operatorname{Van}^{\prime} \subset\left\{l^{\prime} \mid\left(l^{\prime}, E_{v}\right) \leq 1\right.$ for all $\left.v\right\}$; in general $V a n^{\prime} \not \subset \mathcal{S}^{\prime}$ (e.g. for rational singularities each $\left.E_{v} \in V_{a n^{\prime}}\right)$, furthermore $\mathcal{S}_{\text {dom }}^{\prime} \subset V a n^{\prime}$,

(ii) $0 \in V a n^{\prime}$ iff $L$ is rational or elliptic,

(iii) $V a n^{\prime}$ is not necessarily a semigroup $\left(2 E_{v} \notin \operatorname{Van}^{\prime}\right.$ if $|\mathcal{V}|>1, c f$. (i)),

(iv) $\operatorname{Van}^{\prime}$ is closed to the $\mathcal{S}^{\prime}$-action,

(v) $\operatorname{Van}^{\prime}$ is min-stable,

(vi) $V a n^{\prime} \backslash \mathcal{S}^{\prime}$ might have infinitely many elements (e.g. if $E_{v} \in \operatorname{Van}^{\prime}$ then $E_{v}+\mathcal{S}^{\prime} \subset$ Van'too),

(vii) $V a n^{\prime}$ is not necessarily in the first quadrant, however $V a n^{\prime} \cap L$ is in the first quadrant for a minimal resolution (hence for $\mathcal{L}$ generic and with $c_{1}(\mathcal{L}) \in L$, the vanishing $h^{1}(\widetilde{X}, \mathcal{L})$ implies $\left.c_{1}(\mathcal{L}) \leq 0\right)$.

Proof For $(i)$ take $l=E_{v}$ in (5.4.1), and check $h^{1}\left(\mathcal{O}\left(-E_{v}\right)\right)=0$ for rational germs. For $(i v)-(v)$ repeat the arguments from the proof of 4.1.7. For (vii) note that if the graph consists of a $(-1)$ (resp. (-2)) vertex then $-E$ (resp. $-E / 2)$ is in $V a n^{\prime}$. On the other hand, if $-l^{\prime}=x_{1}-x_{2}$, where $x_{1}, x_{2} \in L_{\geq 0}$ have no common $E_{v}$ in their supports, then $\chi\left(-l^{\prime}\right) \leq \chi\left(-l^{\prime}+x_{2}\right)$ implies $\chi\left(-x_{2}\right) \leq 0$. But, in a minimal graph if $\chi(-x) \leq 0$ and $x \geq 0$ then $x=0$. Indeed, take $E_{v} \subset|x|$ such that $\left(E_{v}, x\right)<0$. Then $\chi\left(-x+E_{v}\right) \leq \chi(-x) \leq 0$. If we continue the procedure, in the last step we get $\chi\left(-E_{w}\right) \leq 0$ for some $w$, a fact which can happen only if $E_{w}$ is a (-1)-curve.

Remark 5.4.3 In Theorem 5.3.1 (see also Corollary 5.3.4 too) the set of 'generic' line bundles $\mathcal{L} \in \mathrm{Pic}^{l^{\prime}}(Z)$ which satisfy (5.3.2) with equality is not explicit. There exists an open Zariski set for which (5.3.2) holds with equality, but this usually is not the complement of $\operatorname{im}\left(c^{l^{\prime}}\right)$. In other words, the complement of $\operatorname{im}\left(c^{l^{\prime}}\right)$ might have a nontrivial stratification according to the values of $h^{1}(Z, \mathcal{L})$, and the Zariski open strata corresponds to the 'generic' bundles of Theorem 5.3.1.

Indeed, take the graph $\Gamma_{1}$ from Example 3.4.2, and consider the splice quotient analytic structure on it (for details see e.g. [40]). In particular, $p_{g}=3$. Set $Z \gg 0$ (e.g. $\left.Z=Z_{K}\right)$, and $\mathcal{L}:=\mathcal{O}_{Z}\left(-Z_{\text {min }}\right)$. Since $h^{1}\left(\mathcal{O}_{Z_{\text {min }}}\right)=2$ and $h^{1}\left(\tilde{X}, \mathcal{O}\left(-Z_{\text {min }}\right)\right)=1$, one also has $h^{1}(Z, \mathcal{L})=1$. Note also that the maximal ideal cycle $Z_{\text {max }}$ is $2 Z_{\text {min }}$, hence $\mathcal{L} \notin \operatorname{im}\left(c^{-Z_{\text {min }}}\right)$. On the other hand, $\min \chi=\chi\left(Z_{\text {min }}\right)=-1$, hence $h^{1}\left(Z, \mathcal{L}_{\text {gen }}\right)=0$ for generic bundles $\mathcal{L}_{g e n} \in \mathrm{Pic}^{-Z_{\min }}(Z)$. Hence, the complement of $\operatorname{im}\left(c^{-Z_{\text {min }}}\right)$ has a non-trivial $h^{1}$-stratification.

\subsection{The cohomology cycle of line bundles}

If $(X, o)$ is a singularity with $p_{g}>0$, then its cohomology cycle (associated with a fixed resolution $\phi$ ) is the unique minimal cycle $Z_{c o h} \in L_{>0}$ such that $p_{g}=h^{1}\left(Z_{c o h}, \mathcal{O}_{\tilde{X}}\right)$. We extend this definition as follows.

Proposition 5.5.1 (a) Fix a line bundle $\mathcal{L} \in \operatorname{Pic}(\widetilde{X})$ with $h^{1}(\tilde{X}, \mathcal{L})>0$. The set $L_{\mathcal{L}}:=\left\{l \in L_{>0}: h^{1}(l, \mathcal{L})=h^{1}(\widetilde{X}, \mathcal{L})\right\}$ has a unique minimal element, denoted by $Z_{\text {coh }}(\mathcal{L})$, called the cohomological cycle of $\mathcal{L}$ (and of $\left.\phi\right)$. It has the property that $h^{1}(l, \mathcal{L})<h^{1}(\tilde{X}, \mathcal{L})$ for any $l \ngtr Z_{\text {coh }}(\mathcal{L})(l>0)$.

(b) Fix $Z>0$ and $\mathcal{L} \in \operatorname{Pic}(Z)$ with $h^{1}(Z, \mathcal{L})>0$. The set $L_{Z, \mathcal{L}}:=\{l \in L, 0<l \leq$ $\left.Z: h^{1}(l, \mathcal{L})=h^{1}(Z, \mathcal{L})\right\}$ has a unique minimal element, denoted by $Z_{\text {coh }}(Z, \mathcal{L})$, 
called the cohomological cycle of $(Z, \mathcal{L})$. It has the property that $h^{1}(l, \mathcal{L})<$ $h^{1}(Z, \mathcal{L})$ for any $l \ngtr Z_{\text {coh }}(Z, \mathcal{L})(0<l \leq Z)$.

Proof The proof of $[57,4.8]$, valid for $\mathcal{O}_{\widetilde{X}}$, can be adopted to this situation as well. If $h^{1}(\widetilde{X}, \mathcal{L})=0$, then by convention $Z_{\text {coh }}(\mathcal{L})=0$.

Corollary 5.5.2 (a) For any $l^{\prime} \in L^{\prime}$ consider the set

$$
L_{l^{\prime}}:=\left\{l_{\min } \in L_{\geq 0} \mid \chi\left(-l^{\prime}+l_{\min }\right)=\min _{l \in L_{\geq 0}} \chi\left(-l^{\prime}+l\right)\right\} .
$$

Then $L_{l^{\prime}}$ has a unique minimal element $Z_{c o h}\left(l^{\prime}\right)$, which coincides with the cohomological cycle of any generic $\mathcal{L} \in \operatorname{Pic}^{l^{\prime}}(\widetilde{X})$.

(b) For any $Z>0$ and $l^{\prime} \in L^{\prime}$ consider the set

$$
L_{Z, l^{\prime}}:=\left\{l_{\min } \in L, 0 \leq l_{\min } \leq Z, \mid \chi\left(-l^{\prime}+l_{\min }\right)=\min _{0 \leq l \leq Z, l \in L} \chi\left(-l^{\prime}+l\right)\right\} .
$$

Then $L_{Z, l^{\prime}}$ has a unique minimal element $Z_{c o h}\left(Z, l^{\prime}\right)$, which coincides with the cohomological cycle of any generic $\mathcal{L} \in \mathrm{Pic}^{l^{\prime}}(Z)$.

Proof Combine Theorem 5.3.1 and Proposition 5.5.1.

Corollary 5.5.3 (1) Elements of type $-l^{\prime}+Z_{\text {coh }}\left(l^{\prime}\right)\left(l^{\prime} \in L^{\prime}\right)$ belong to Van'.

(2) If $-l^{\prime} \leq-l^{\prime \prime}$ then $-l^{\prime}+Z_{c o h}\left(l^{\prime}\right) \leq-l^{\prime \prime}+Z_{c o h}\left(l^{\prime \prime}\right)$ as well. Furthermore, if $-l^{\prime} \leq-l^{\prime \prime} \leq-l^{\prime}+Z_{c o h}\left(l^{\prime}\right)$ then $-l^{\prime}+Z_{c o h}\left(l^{\prime}\right)=-l^{\prime \prime}+Z_{c o h}\left(l^{\prime \prime}\right)$.

Example 5.5.4 Assume that $L$ is numerically Gorenstein (that is, $Z_{K} \in L$ ). Then by [21, Lemma 6] (and $\chi(l)=\chi\left(Z_{K}-l\right)$ ) one gets $Z_{\text {coh }}\left(l^{\prime}=0\right) \leq Z_{K} / 2$.

\subsection{The dimension of $\operatorname{im}(c)$}

For an arbitrary element $\mathcal{L}$ of the image $\operatorname{im}\left(c: \mathrm{ECa}^{l^{\prime}}(Z) \rightarrow \mathrm{Pic}^{l^{\prime}}(Z)\right)$ one has $\operatorname{dimim}(c)+\operatorname{dim} c^{-1}(\mathcal{L}) \geq \operatorname{dim} \operatorname{ECa}^{l^{\prime}}(Z)=\left(l^{\prime}, Z\right)$, with equality whenever $\mathcal{L}$ is a generic element of the image $\operatorname{im}(c)$. This combined with Lemma 3.1.7(b) gives the following.

Proposition 5.6.1 For any $\mathcal{L} \in \operatorname{im}\left(c^{l^{\prime}}\right) \subset \operatorname{Pic}^{l^{\prime}}(Z)$ one has

$$
h^{1}(Z, \mathcal{L}) \geq h^{1}\left(\mathcal{O}_{Z}\right)-\operatorname{dim}\left(\operatorname{im}\left(c^{l^{\prime}}\right)\right)=\operatorname{codim}\left(\operatorname{im}\left(c^{l^{\prime}}\right)\right) .
$$

In (5.6.2) equality holds whenever $\mathcal{L}$ is generic in the image of $c$ (that is, generic with the property $\left.H^{0}(Z, \mathcal{L})_{\text {reg }} \neq \emptyset\right)$. This fact and Theorem 5.3.1 applied for the generic element of $\operatorname{im}(c)$ imply

$$
\operatorname{codim}\left(\operatorname{im}\left(c^{l^{\prime}}\right)\right) \geq \chi\left(-l^{\prime}\right)-\min _{0 \leq l \leq Z} \chi\left(-l^{\prime}+l\right) .
$$

Furthermore, if $c^{l^{\prime}}$ is not dominant then the inequality in (5.6.3) is strict. 
In general, the codimension of $\operatorname{im}(c)$ cannot be characterized topologically. Indeed, take e.g. $l^{\prime}=0$, then $\operatorname{im}(c)$ is a point with codimension $h^{1}\left(\mathcal{O}_{Z}\right)$. Moreover, by Example 3.4.1, the dimension of $\operatorname{im}(c)$ is not topological either.

\subsection{Upper bounds for $h^{1}(Z, \mathcal{L})$}

Theorem 5.3.1 and Corollary 5.3.4 provide sharp lower bounds for $h^{1}(Z, \mathcal{L})$ and $h^{1}(\widetilde{X}, \mathcal{L})$. A possible upper bound is given by the next proposition.

Proposition 5.7.1 Fix $Z>0$ and an arbitrary $\mathcal{L} \in \operatorname{Pic}(Z)$ with $l^{\prime}=c_{1}(\mathcal{L}) \in-\mathcal{S}^{\prime}$.

(a) If $h^{0}(Z, \mathcal{L})=0$ then $h^{1}(Z, \mathcal{L}) \leq-\chi(Z)<h^{1}\left(\mathcal{O}_{Z}\right)$.

(b) If $H^{0}(Z, \mathcal{L})_{\text {reg }} \neq \emptyset$ then $h^{1}(Z, \mathcal{L}) \leq h^{1}\left(\mathcal{O}_{Z}\right)$.

(c) In general, if $h^{0}(Z, \mathcal{L}) \neq 0$ then

$$
\begin{aligned}
h^{1}(Z, \mathcal{L}) & \leq \max _{0 \leq l \leq Z}\left\{h^{1}\left(\mathcal{O}_{Z-l}\right)+\chi\left(-l^{\prime}\right)-\chi\left(-l^{\prime}+l\right)\right\} \\
& \leq h^{1}\left(\mathcal{O}_{Z}\right)+\chi\left(-l^{\prime}\right)-\min _{0 \leq l \leq Z} \chi\left(-l^{\prime}+l\right) .
\end{aligned}
$$

In particular, by (5.3.2) and (5.7.2), $h^{1}(Z, \mathcal{L})$ takes values in an interval of length (at most) $h^{1}\left(\mathcal{O}_{Z}\right)$.

Note that $h^{1}\left(\mathcal{O}_{Z}\right) \leq \max _{0 \leq l \leq Z}\left\{h^{1}\left(\mathcal{O}_{Z-l}\right)+\chi\left(-l^{\prime}\right)-\chi\left(-l^{\prime}+l\right)\right\}$ (take $\left.l=0\right)$. Hence $(b)$ gives a better bound than $(c)$ whenever $H^{0}(Z, \mathcal{L})_{\text {reg }} \neq \emptyset$. (Examples with $h^{1}(Z, \mathcal{L}) \not \leq h^{1}\left(\mathcal{O}_{Z}\right)$ exist even for $l^{\prime}=0$, see e.g. Example 8.2.4 in part II [32], when we will treat the generic analytic structures).

Furthermore, $(c)$ for $l^{\prime}=0$ reads as $h^{1}(Z, \mathcal{L}) \leq \max _{0 \leq l \leq Z}\left\{h^{1}\left(\mathcal{O}_{Z-l}\right)-\chi(l)\right\}$, which for $Z=Z_{K} \in L$ transforms into $h^{1}\left(Z_{K}, \mathcal{L}\right) \leq \max _{0 \leq l \leq Z_{K}}\left\{h^{1}\left(\mathcal{O}_{l}\right)-\chi(l)\right\}$ (use $\left.\chi\left(Z_{K}-l\right)=\chi(l)\right)$.

$\operatorname{Proof}\left(\right.$ a) $h^{1}(Z, \mathcal{L})=-\chi(Z, \mathcal{L})=-\chi(Z)-\left(Z, l^{\prime}\right) \leq-\chi(Z)=-h^{0}\left(\mathcal{O}_{Z}\right)+$ $h^{1}\left(\mathcal{O}_{Z}\right)$.

(b) Multiplication by a generic $s \in H^{0}(Z, \mathcal{L})$ gives an exact sequence of sheaves $0 \rightarrow \mathcal{O}_{Z} \rightarrow \mathcal{L} \rightarrow \mathcal{F} \rightarrow 0$, where $\mathcal{F}$ is Stein. Hence $H^{1}\left(\mathcal{O}_{Z}\right) \rightarrow H^{1}(Z, \mathcal{L})$ is onto and $h^{1}(Z, \mathcal{L}) \leq h^{1}\left(\mathcal{O}_{Z}\right)$.

(c) If $l$ is the fixed divisor of $\mathcal{L}$ supported on $E$, then from the exact sequence $0 \rightarrow$ $\left.\left.\mathcal{L}(-l)\right|_{Z-l} \rightarrow \mathcal{L} \rightarrow \mathcal{L}\right|_{l} \rightarrow 0$ we get $h^{1}(\mathcal{L})=h^{1}(Z-l, \mathcal{L}(-l))-\chi\left(\left.\mathcal{L}\right|_{l}\right)$, and $\left.\mathcal{L}(-l)\right|_{Z-l}$ has no fixed components. Hence $h^{1}(Z-l, \mathcal{L}(-l)) \leq h^{1}\left(\mathcal{O}_{Z-l}\right)$ by $(b)$.

Remark 5.7.3 The inequality $h^{1}(Z, \mathcal{L}) \leq h^{1}\left(\mathcal{O}_{Z}\right)$, valid for the case when $\mathcal{L}$ has no fixed components, has the following geometric interpretation, cf. (3.1.8): $h^{1}\left(\mathcal{O}_{Z}\right)-$ $h^{1}(Z, \mathcal{L})=\operatorname{codim}\left(c^{-1}(\mathcal{L}) \subset \mathrm{ECa}^{l^{\prime}}\right) \geq 0$. The inequality for $\mathcal{L}=\mathcal{O}(-l), l \in L_{>0}$, was already proved in [51, Th. 3.1]. 


\subsection{The $h^{1}$-stratification of Pic ${ }^{\prime \prime}(Z)$}

Fix $Z>0, l^{\prime} \in-\mathcal{S}^{\prime}$ and $k \in \mathbb{Z}$ with

$$
\chi\left(-l^{\prime}\right)-\min _{0 \leq l \leq Z} \chi\left(-l^{\prime}+l\right) \leq k \leq h^{1}\left(\mathcal{O}_{Z}\right)+\chi\left(-l^{\prime}\right)-\min _{0 \leq l \leq Z} \chi\left(-l^{\prime}+l\right) .
$$

Definition 5.8.1 For any $l^{\prime}$ and $k$ as above we set

$$
W_{l^{\prime}, k}:=\left\{\mathcal{L} \in \operatorname{Pic}^{l^{\prime}}(Z): h^{1}(Z, \mathcal{L})=k\right\}
$$

From the semicontinuity Lemma 5.2.1 we automatically have for the closure $\overline{W_{l^{\prime}, k}}$

$$
\overline{W_{l^{\prime}, k}} \subset\left\{\mathcal{L} \in \operatorname{Pic}^{l^{\prime}}(Z): h^{1}(Z, \mathcal{L}) \geq k\right\}
$$

These sets constitute the analogs of the Brill-Noether strata defined for projective curves by the Brill-Noether theory, see $[1,13]$ and the references therein.

Lemmas 5.6.1 and 3.3.2 have the following consequences.

Corollary 5.8.4 Fix $l^{\prime} \in-\mathcal{S}^{\prime}$. Then $\operatorname{im}\left(c^{l^{\prime}}\right) \subset \overline{W_{l^{\prime}, \operatorname{codim} \operatorname{im}\left(c^{l^{\prime}}\right)}}$. Furthermore, the set of critical bundles of $c^{l^{\prime}}$ are included in $\overline{W_{l^{\prime}, \operatorname{codimim}\left(c^{l^{\prime}}\right)+1}}$.

Example 5.8.5 If the fibers of $c^{l^{\prime}}$ over $\operatorname{im}\left(c^{l^{\prime}}\right)$ are not equidimensional, then $\operatorname{im}\left(c^{l^{\prime}}\right)$ consists of more strata of type $W_{l^{\prime}, k}$ (see e.g. Example 3.4.3). But, even if the fibers over $\operatorname{im}\left(c^{l^{\prime}}\right)$ are equidimensional, hence $\operatorname{im}\left(c^{l^{\prime}}\right)$ consists of only one stratum, it can happen that $c^{l^{\prime}}$ is not a (topological) locally trivial fibration over $\operatorname{im}\left(c^{l^{\prime}}\right)$, see e.g. Example 3.4.4. In particular, $c^{l^{\prime}}$ over a strata $W_{l^{\prime}, k}$ usually is not a (topological) locally trivial fibration.

\section{6 'Multiplicative' structures. The 'stable' im $\left(c^{\prime \prime}\right)$}

\subsection{Monoid structure of divisors}

In this section we will exploit the additional natural additive structure $s^{l_{1}^{\prime}, l_{2}^{\prime}}(Z)$ : $\operatorname{ECa}^{l_{1}^{\prime}}(Z) \times \mathrm{ECa}^{l_{2}^{\prime}}(Z) \rightarrow \mathrm{ECa}^{l_{1}^{\prime}+l_{2}^{\prime}}(Z)\left(l_{1}^{\prime}, l_{2}^{\prime} \in-\mathcal{S}^{\prime}\right)$ provided by the sum of the divisors. (Sometimes we will abridge $s_{1}^{\prime}, l_{2}^{\prime}(Z)$ as $s$.)

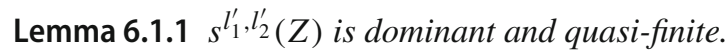

Proof An effective divisor decomposes in finitely many ways, hence the quasifiniteness follows. Since the dimensions of the source and the target are equal, cf. Theorem 3.1.10, $s$ is dominant.

In general, $s$ is not surjective. E.g., in Example 3.4.4, the elements of $c^{-1}\left(E_{1} \cap E_{2}\right)=$ $\mathbb{C}^{*}$ are not in the image of $s_{1}^{*}, E_{2}^{*}(Z)$. 
There is a parallel multiplication $\operatorname{Pic}^{l_{1}^{\prime}}(Z) \times \operatorname{Pic}_{2}^{l_{2}^{\prime}}(Z) \rightarrow \operatorname{Pic}^{\prime}+l_{2}^{\prime}(Z),\left(\mathcal{L}_{1}, \mathcal{L}_{2}\right) \mapsto$ $\mathcal{L}_{1} \otimes \mathcal{L}_{2}$. Clearly, $c^{l_{1}^{\prime}+l_{2}^{\prime}} \circ s^{l_{1}^{\prime}, l_{2}^{\prime}}=c^{l_{1}^{\prime}} \otimes c^{l_{2}^{\prime}}$ in Pic $^{l_{1}^{\prime}+l_{2}^{\prime}}$. In the next discussions we replace $c^{l^{\prime}}$ by the composition

$$
\widetilde{c}^{l^{\prime}}: \mathrm{ECa}^{l^{\prime}}(Z) \stackrel{c^{l^{\prime}}}{\longrightarrow} \operatorname{Pic}^{l^{\prime}}(Z) \stackrel{\mathcal{O}_{Z\left(-l^{\prime}\right)}}{\longrightarrow} \operatorname{Pic}^{0}(Z)
$$

where the second map is the multiplication by the natural line bundle $\mathcal{O}_{Z}\left(-l^{\prime}\right)$. Since $\mathcal{O}_{Z}\left(l_{1}^{\prime}+l_{2}^{\prime}\right)=\mathcal{O}_{Z}\left(l_{1}^{\prime}\right) \otimes \mathcal{O}_{Z}\left(l_{2}^{\prime}\right)$ we also have $\widetilde{c}^{\prime}+l_{2}^{\prime} \circ s^{l_{1}^{\prime}, l_{2}^{\prime}}=\widetilde{c}^{\prime} \otimes \widetilde{c}^{\prime}$ in Pic ${ }^{0}$. After identification of $\mathrm{Pic}^{0}$ with (the additive) $H^{1}\left(\mathcal{O}_{Z}\right)$, this reads as $\widetilde{c}_{1}^{\prime}+l_{2}^{\prime} \circ s^{l_{1}^{\prime}, l_{2}^{\prime}}=\widetilde{c}^{l_{1}^{\prime}}+\widetilde{c}^{\prime}{ }_{2}$ in $H^{1}\left(\mathcal{O}_{Z}\right)$. The advantage of this new map is that it collects all the images of the effective Cartier divisors in a single vector space $H^{1}\left(\mathcal{O}_{Z}\right)$. Lemma 6.1.1 and the construction imply

$$
\operatorname{im}\left(\widetilde{c}_{1}^{\prime}\right)+\operatorname{im}\left(\widetilde{c}_{2}^{\prime}\right) \subset \operatorname{im}\left(\widetilde{c}_{1}^{\prime}+l_{2}^{\prime}\right) \subset \overline{\operatorname{im}\left(\widetilde{c}_{1}^{\prime}\right)+\operatorname{im}\left(\widetilde{c}_{2}^{\prime}\right)}
$$

Definition 6.1.3 For any $l^{\prime} \in-\mathcal{S}^{\prime}$ let $A_{Z}\left(l^{\prime}\right)$ (if there is no confusion, $A\left(l^{\prime}\right)$ ) be the smallest dimensional affine subspace of $H^{1}\left(\mathcal{O}_{Z}\right)$ which contains $\operatorname{im}\left(\widetilde{c}^{\prime}\right)$. Let $V_{Z}\left(l^{\prime}\right)$ be the parallel vector subspace of $H^{1}\left(\mathcal{O}_{Z}\right)$, the translation of $A_{Z}\left(l^{\prime}\right)$ to the origin.

Remark 6.1.4 From this definition follows that $\operatorname{dim} V_{Z}\left(l^{\prime}\right)$ is greater than or equal to the dimension of the Zariski tangent space at any $\mathcal{L} \in \overline{\operatorname{im}\left(c^{l^{\prime}}(Z)\right)}$; in particular, $\left.\operatorname{dim} V_{Z}\left(l^{\prime}\right)\right) \geq \operatorname{dimim}\left(c^{l^{\prime}}(Z)\right)$. Hence, by (5.6.2) one also has $\operatorname{dim} V_{Z}\left(l^{\prime}\right) \geq h^{1}\left(\mathcal{O}_{Z}\right)-$ $h^{1}(Z, \mathcal{L})$ for any $\mathcal{L} \in \operatorname{im}\left(c^{l^{\prime}}(Z)\right)$.

Example 6.1.5 In general, $\operatorname{im}\left(\widetilde{c}^{\prime}\right) \varsubsetneqq A_{Z}\left(l^{\prime}\right)$; take e.g. the first case of Example 3.4.2, when $\operatorname{dim} \operatorname{im}\left(c^{l^{\prime}}\right)=1$ and $A_{Z}\left(l^{\prime}\right)=\mathbb{C}^{2}$. (The fact that $A_{Z}\left(l^{\prime}\right)=\mathbb{C}^{2}$ can be deduced in the following way as well. $c^{n l^{\prime}}$ is dominant for $n \gg 1$, hence $A_{Z}\left(n l^{\prime}\right)=\mathbb{C}^{2}$. But $V_{Z}\left(l^{\prime}\right)=V_{Z}\left(n l^{\prime}\right)$, see e.g. the next Lemma.)

Using (6.1.2) one obtains the following properties of the spaces $\left\{A_{Z}\left(l^{\prime}\right)\right\}_{l^{\prime}}$ of $H^{1}\left(\mathcal{O}_{Z}\right)$ :

Lemma 6.1.6 (a) $A_{Z}\left(l_{1}^{\prime}+l_{2}^{\prime}\right)=A_{Z}\left(l_{1}^{\prime}\right)+A_{Z}\left(l_{2}^{\prime}\right):=\left\{a_{1}+a_{2}: a_{i} \in A_{Z}\left(l_{i}^{\prime}\right\} ;\right.$ in particular, $V_{Z}\left(l_{1}^{\prime}\right) \subset V_{Z}\left(l_{2}^{\prime}\right)$ whenever $l_{1}^{\prime} \leq l_{2}^{\prime}$ and $V_{Z}\left(n l^{\prime}\right)=V_{Z}\left(l^{\prime}\right)$ for any $n \geq 1$.

(b) For any $-l^{\prime}=\sum_{v} a_{v} E_{v}^{*} \in \mathcal{S}^{\prime}$ let the $E^{*}$-support of $l^{\prime}$ be $I\left(l^{\prime}\right):=\left\{v: a_{v} \neq 0\right\}$. Then $V_{Z}\left(l^{\prime}\right)$ depends only on $I\left(l^{\prime}\right)$.

E.g., if $I\left(l^{\prime}\right)=\mathcal{V}$, then $c^{n l^{\prime}}$ is dominant for any $n \gg 1$ (use Theorem 4.1.1(3).) Hence, $V_{Z}\left(l^{\prime}\right)=V_{Z}\left(n l^{\prime}\right)=H^{1}\left(\mathcal{O}_{Z}\right)$.

Proof (b) $V_{Z}\left(l^{\prime}\right) \subset V_{Z}\left(l^{\prime}+n E_{v}^{*}\right) \subset V_{Z}\left(l^{\prime}\right)+V_{Z}\left(n E_{v}^{*}\right) \subset V_{Z}\left(l^{\prime}\right)+V_{Z}\left(E_{v}^{*}\right) \subset V_{Z}\left(l^{\prime}\right)$ for $v \in I\left(l^{\prime}\right)$.

Definition 6.1.7 (a) 6.1.6(b) motivates to use the notation $V_{Z}(I)$ for $V_{Z}\left(l^{\prime}\right)$ whenever $I=I\left(l^{\prime}\right)$.

Hence Lemma 6.1.6(a) reads as $V_{Z}\left(I_{1} \cup I_{2}\right)=V_{Z}\left(I_{1}\right)+V_{Z}\left(I_{2}\right)$. 
(b) If $Z_{2} \geq Z_{1}$, then the restriction (cf. 3.1) satisfies $r\left(V_{Z_{2}}\left(l^{\prime}\right)\right)=V_{Z_{1}}\left(l^{\prime}\right)$, hence $\operatorname{dim} V_{Z_{2}}\left(l^{\prime}\right) \geq \operatorname{dim} V_{Z_{1}}\left(l^{\prime}\right)$ and the pair $V_{Z}\left(l^{\prime}\right) \subset H^{1}\left(\mathcal{O}_{Z}\right)$ stabilizes as $Z$ increases. Set $\left(V_{\widetilde{X}}\left(l^{\prime}\right) \subset H^{1}\left(\mathcal{O}_{\widetilde{X}}\right)\right)$ for $\lim _{\leftarrow}\left(V_{Z}\left(l^{\prime}\right) \subset H^{1}\left(\mathcal{O}_{Z}\right)\right)$ and $\left(V_{\tilde{X}}(I) \subset H^{1}\left(\mathcal{O}_{\tilde{X}}\right)\right):=\lim _{\leftarrow}\left(V_{Z}(I) \subset H^{1}\left(\mathcal{O}_{Z}\right)\right)$.

Remark 6.1.8 The multiplicative structure-that is, the general properties what must be satisfied by $\widetilde{c}^{n l^{\prime}}$ for a certain $n>1$-imposes strong hidden properties for the original map $\widetilde{c}^{l^{\prime}}: \mathrm{ECa}^{l^{\prime}}(Z) \rightarrow \operatorname{Pic}^{0}(Z)=H^{1}\left(\mathcal{O}_{Z}\right)$ as well. Let us exemplify this via the following case. Assume e.g. that $Z \geq E$ and $\mathrm{ECa}^{l^{\prime}}(Z)$ is 1-dimensional. Then $\mathrm{ECa}^{l^{\prime}}(Z)$ can be identified with some $E_{v}^{r e g}:=E_{v} \backslash \cup_{w \neq v} E_{w}$. Therefore, the symmetric product $\mathrm{ECa}^{l^{\prime}}(Z)^{\times n} / \mathfrak{S}_{n}$ (where $\mathfrak{S}_{n}$ is the permutation group of $n$ letters) embeds as a Zariski open set into $\mathrm{ECa}^{n l^{\prime}}(Z)$. Hence, by Lemma 3.1.7, the generic fibers of the restriction of $\widetilde{c}^{n l^{\prime}}\left(\mathrm{ECa}^{l^{\prime}}(Z)^{\times n} / \mathfrak{S}_{n} \rightarrow H^{1}\left(\mathcal{O}_{Z}\right),\left[D_{1}, \ldots, D_{n}\right] \mapsto \sum_{i} \widetilde{c}^{l^{\prime}}\left(D_{i}\right)\right)$ must be irreducible. This fact imposes serious restrictions for the original map $\widetilde{c}^{\prime}$ as well.

E.g., $\mathbb{C} \rightarrow \mathbb{C}^{2}, t \mapsto\left(t, t^{4}\right)$ cannot be birational equivalent with a certain $\widetilde{c}^{\prime}$. Indeed, its 'double', $\mathbb{C}^{\times 2} / \mathfrak{S}_{2} \rightarrow \mathbb{C}^{2},(t, s) \mapsto\left(t+s, t^{4}+s^{4}\right)$, rewritten in terms of elementary symmetric functions reads as $\mathbb{C}^{2} \rightarrow \mathbb{C}^{2},\left(\sigma_{1}, \sigma_{2}\right) \mapsto\left(\sigma_{1}, \sigma_{1}^{4}-4 \sigma_{2} \sigma_{1}^{2}+2 \sigma_{2}^{2}\right)$, which has non-irreducible generic fibers.

By the next theorem, $V_{Z}\left(l^{\prime}\right)=H^{1}\left(\mathcal{O}_{Z}\right)$ if and only if $c^{n l^{\prime}}$ is dominant for $n \gg 1$; and in 6.3 we will characterize those cases when $V_{Z}\left(l^{\prime}\right)=0$. But besides these two limit situations the construction provides a rather complex linear subspace arrangement $\left\{V_{Z}\left(l^{\prime}\right)\right\}_{l^{\prime}}$, which, in general, contains deep analytic information about $(X, o)$.

Theorem 6.1.9 Fix $l^{\prime} \in-\mathcal{S}^{\prime}$ and $Z>0$ as above. Then for $n \gg 1$ the following facts hold.

(a) The image of $\widetilde{c}^{n l^{\prime}}$ is the affine subspace $A_{Z}\left(n l^{\prime}\right)$ of $H^{1}\left(\mathcal{O}_{Z}\right)$ (a translated of $\left.A_{Z}\left(l^{\prime}\right)\right)$.

(b) All the (non-empty) fibers of $\widetilde{c}^{n l^{\prime}}$ have the same dimension.

In particular, for any $\mathcal{L} \in \mathrm{Pic}^{n l^{\prime}}(Z)$ without fixed components (and $n \gg 1$ ) one has

$$
h^{1}(Z, \mathcal{L})=h^{1}\left(\mathcal{O}_{Z}\right)-\operatorname{dim} V_{Z}\left(l^{\prime}\right)=\operatorname{codim}\left(V_{Z}\left(l^{\prime}\right) \subset H^{1}\left(\mathcal{O}_{Z}\right)\right)
$$

(c) Let $I \subset \mathcal{V}$ be the $E^{*}$-support of $l^{\prime}$. Decompose $Z$ as $\left.Z\right|_{I}+\left.Z\right|_{\mathcal{V} \backslash I}$ according to the supports $I$ and $\mathcal{V} \backslash I$. Then for all $\mathcal{L} \in \mathrm{Pic}^{n l^{\prime}}(Z)$ without fixed components (and $n \gg 1) h^{1}(Z, \mathcal{L})$ depends only on the $E^{*}$-support I of $l^{\prime}$ :

$$
h^{1}(Z, \mathcal{L})=h^{1}\left(\mathcal{O}_{Z \mid \mathcal{V} \backslash I}\right) .
$$

Hence, by (6.1.10),

$$
\operatorname{dim} V_{Z}(I)=h^{1}\left(\mathcal{O}_{Z}\right)-h^{1}\left(\mathcal{O}_{Z \mid \mathcal{V} \backslash I}\right) .
$$

In particular, if $\left(\tilde{X} / E_{\mathcal{V} \backslash I}, o \mathcal{V} \backslash I\right)$ denotes the multi-germ (the disjoint union of singularities) obtained by contracting the connected components of $E_{\mathcal{V} \backslash I}$ in $\widetilde{X}$, then for $Z \gg 0$ we obtain 


$$
\operatorname{dim} V_{Z}(I)=p_{g}(X, o)-p_{g}\left(\tilde{X} / E_{\mathcal{V} \backslash I}, o \mathcal{V} \backslash I\right)
$$

Therefore, $V_{Z}(I)=H^{1}\left(\mathcal{O}_{Z}\right)=\mathbb{C}^{p_{g}(X, o)}$, if and only if $\Gamma \backslash I$ is a disjoint union of rational graphs.

(d) With the notations of $(c), V_{Z}(I)=\operatorname{ker}\left(H^{1}\left(\mathcal{O}_{Z}\right) \rightarrow H^{1}\left(\mathcal{O}_{Z \mid \mathcal{V} \backslash I}\right)\right)$.

(e) Any $\mathcal{L} \in \operatorname{Pic}^{n l^{\prime}}(Z)$ without fixed components is generated by global sections.

Remark 6.1.14 (a) In (6.1.10) $h^{1}(Z, \mathcal{L})>-\chi(Z, \mathcal{L})\left(\right.$ since $\left.h^{0}(Z, \mathcal{L})>0\right)$, which gives a topological lower bound for $\operatorname{codim}\left(V_{Z}\left(l^{\prime}\right) \subset H^{1}\left(\mathcal{O}_{Z}\right)\right)$.

(b) (6.1.13) generalizes the ' $p_{g}$-additivity formula' of Okuma [49], which was proved for splice quotient singularities, for details see 9.3. Note that the present formula is valid for any singularity.

(c) Part (a) of Theorem 6.1.9 is equivalent (by a similar argument as the proof of Lemma $6.1 .6(b)$ ) by the following statement: $\left(\mathrm{a}^{\prime}\right)$ If $-l^{\prime}=\sum_{v \in I} a_{v} E_{v}^{*}$ with $a_{v} \gg 0$ (but no other relations between them), then the image of $\widetilde{c}^{\prime}$ is an affine subspace, a translated of $V_{Z}(I)$.

(d) Parts $(b)-(c)$ of Theorem 6.1.9 imply that $\operatorname{im}\left(c^{n l^{\prime}}\right)$ (for $n \gg 1$ ) is closed and consists of only one $h^{1}$-strata: $\operatorname{im}\left(c^{n l^{\prime}}\right)=W_{n l^{\prime}, h^{1}\left(\mathcal{O}_{Z}\right)-\operatorname{dim} V_{Z}(I)}$.

Proof of Theorem 6.1.9 (a) Write $A\left(l^{\prime}\right)$ as $a+V\left(l^{\prime}\right)$ for some $a \in A\left(l^{\prime}\right)$. Then by (6.1.2) $\operatorname{im}\left(\widetilde{c}^{n l^{\prime}}\right) \subset n a+V\left(l^{\prime}\right)$. We have to show that for $n \gg 0$ we have equality $\operatorname{im}\left(\widetilde{c}^{n l^{\prime}}\right)=n a+V\left(l^{\prime}\right)$.

We choose smooth points $x_{1}, \ldots, x_{k}$ in $\operatorname{im}\left(\widetilde{c}^{\prime}\right)$ such that the tangent spaces $T_{x_{i}} \operatorname{im}\left(\widetilde{c}^{\prime}\right)$, translated to the origin, generate $V\left(l^{\prime}\right)$. Then taking Zariski neighborhoods $U_{i}$ of $x_{i}$ in $\operatorname{im}\left(\widetilde{c}^{\prime}\right)$, we notice that $\sum_{i}\left(-x_{i}+U_{i}\right)$ contains a Zariski open set of $V\left(l^{\prime}\right)$. But $\sum_{i}\left(-x_{i}+U_{i}\right) \subset \sum_{i}\left(-x_{i}+\operatorname{im}\left(\widetilde{c}^{\prime}\right)\right) \subset-\sum_{i} x_{i}+\operatorname{im}\left(\widetilde{c}^{k l^{\prime}}\right) \subset V\left(l^{\prime}\right)$, hence $-\sum_{i} x_{i}+\operatorname{im}\left(\widetilde{c}^{k l^{\prime}}\right)$ contains a Zariski open subset of $V\left(l^{\prime}\right)$. On the other hand, if $U$ is a Zariski open set of a vector space $V$, then $U+U=V$. This shows that $\operatorname{im}\left(\widetilde{c}^{2 k l^{\prime}}\right)$ is an affine space associated with $V\left(l^{\prime}\right)$.

(b) If we replace $l^{\prime}$ by some multiple if it, by part $(a)$ we can assume that $\widetilde{c}^{\prime}$ : $\mathrm{ECa}^{l^{\prime}}(Z) \rightarrow H^{1}\left(\mathcal{O}_{Z}\right)$ has image $A\left(l^{\prime}\right)$. Consider the following diagram (for some $m \in \mathbb{Z}_{>0}$ which will be determined later):

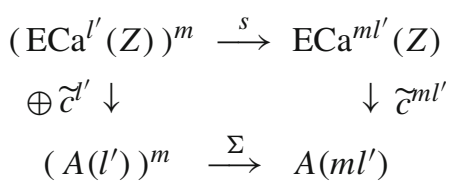

Fix any $x \in A\left(m l^{\prime}\right)$. Since $\oplus \widetilde{c}^{l^{\prime}}$ and $\Sigma$ are surjective, the fiber $\left(\widetilde{c}^{m l^{\prime}}\right)^{-1}(x)$ intersects $\operatorname{im}(s)$ at some point $p$. Since the source and target spaces of $s$ are smooth of the same dimension, by Open Mapping Theorem (see e.g. [14, p. 107]) there exists an (analytic) open neighbourhood $U$ of $p$ (hence intersecting the fiber) contained in $\operatorname{im}(s)$. Hence, using also the quasi-finiteness of $s, \operatorname{dim}\left(\widetilde{c}^{m l^{\prime}}\right)^{-1}(x)=\operatorname{dim}\left(\widetilde{c}^{m l^{\prime}} \circ s\right)^{-1}(x)=$ $\operatorname{dim}\left(\Sigma \circ \oplus \widetilde{c}^{\prime}\right)^{-1}(x)$. Thus, if $\mathbf{x}=\left(x_{1}, \ldots, x_{m}\right)$ are the coordinates in $\left(A\left(l^{\prime}\right)\right)^{m}$, then we have to analyse the set $\left(\oplus \widetilde{c}^{\prime}\right)^{-1}\left\{\mathbf{x}: \sum_{i} x_{i}=x\right\}$ for any fixed $x$.

In $A\left(l^{\prime}\right)$ there is a Zariski open subset $U$, with the following two properties: 
(i) for any $y \in U$, the fiber $\left(\widetilde{c}^{\prime}\right)^{-1}(y)$ has the minimal possible dimension, namely $\operatorname{dim} \mathrm{ECa}^{l^{\prime}}(Z)-\operatorname{dim} A\left(l^{\prime}\right)=\left(l^{\prime}, Z\right)-d\left(l^{\prime}\right)$

(ii) if $F:=A\left(l^{\prime}\right) \backslash U$ is its complement, then $\operatorname{dim}\left(\widetilde{c}^{\prime}\right)^{-1}(F)<\operatorname{dim} \mathrm{ECa}^{l^{\prime}}(Z)=$ $\left(l^{\prime}, Z\right)$.

We stratify $H_{x}:=\left\{\mathbf{x}: \sum_{i} x_{i}=x\right\}$ with the sets $\mathcal{F}_{k}:=\left\{\mathbf{x} \in H_{x}: \#\left\{i: x_{i} \in F\right\}=\right.$ $k$, where $0 \leq k \leq m$. Set also $E \mathcal{F}_{k}:=\left(\oplus \widetilde{c}^{\prime}\right)^{-1}\left(\mathcal{F}_{k}\right)$.

Then $\mathcal{F}_{0}$ is a non-empty open set of $H_{x}$ of dimension $(m-1) d\left(l^{\prime}\right)$, hence $\operatorname{dim} E \mathcal{F}_{0}=$ $(m-1) d\left(l^{\prime}\right)+m\left(\left(l^{\prime}, Z\right)-d\left(l^{\prime}\right)\right)=\left(m l^{\prime}, Z\right)-d\left(l^{\prime}\right)$. Next we estimate the dimensions of the other strata as well.

First, we consider the case $1 \leq k<m$. Then $\mathcal{F}$ is covered by several components according to the position of $I=\left\{i_{1}, \ldots, i_{k}\right\}$ indexing those $x_{i}$ which belong to $F$. Fix suxh a component $\mathcal{F}_{k, I}$, and write $\left(\oplus \widetilde{c}^{\prime}\right)^{-1}\left(\mathcal{F}_{k, I}\right)=E \mathcal{F}_{k, I}$. We consider the projection $p r_{I}: \mathcal{F}_{k, I} \rightarrow \Pi_{I} F, \mathbf{x} \mapsto\left(x_{i_{1}}, \ldots, x_{i_{k}}\right)$, and the lifted one $E \operatorname{Er}_{I}: E \mathcal{F}_{k, I} \rightarrow \Pi_{I}\left(\widetilde{c}^{l^{\prime}}\right)^{-1}(F)$. Note that $E p r_{I}$ is an injection and its target has dimension $\leq k\left(\left(l^{\prime}, Z\right)-1\right)$. Furthermore, the fibers of $E \operatorname{pr}_{I}$ have dimension $(m-k-1) d\left(l^{\prime}\right)+(m-k)\left(\left(l^{\prime}, Z\right)-d\left(l^{\prime}\right)\right)=(m-k)\left(l^{\prime}, Z\right)-d\left(l^{\prime}\right)$. Hence, $\operatorname{dim} E \mathcal{F}_{k, I} \leq(m-k)\left(l^{\prime}, Z\right)-d\left(l^{\prime}\right)+k\left(\left(l^{\prime}, Z\right)-1\right)=\left(m l^{\prime}, Z\right)-d\left(l^{\prime}\right)-k$.

The case $k=m$ is slightly different. Using the injection $\mathcal{F}_{m} \rightarrow \sqcap_{m}\left(\mathcal{c}^{\prime}\right)^{-1}(F)$ we get 'only' $\operatorname{dim} E \mathcal{F}_{m} \leq m\left(\left(l^{\prime}, Z\right)-1\right)$. Therefore, if $m \geq d\left(l^{\prime}\right)$ then we get $\operatorname{dim} E \mathcal{F}_{m} \leq \operatorname{dim} E \mathcal{F}_{0}$. Hence, finally, $\operatorname{dim}\left(\widetilde{c}^{m l^{\prime}}\right)^{-1}(x)=\operatorname{dim} E \mathcal{F}_{0}=$ $\operatorname{dim} \mathrm{ECa}^{m l^{\prime}}(Z)-\operatorname{dim} A\left(m l^{\prime}\right)$.

For (6.1.10) use part $(b)$ and Lemma 5.6.1.

(c) For any $n \gg 1$ and $\mathcal{L} \in \operatorname{im}\left(c^{n l^{\prime}}\right)$ (6.1.10) gives $h^{1}(Z, \mathcal{L})=h^{1}\left(\mathcal{O}_{Z}\right)-d_{Z}\left(l^{\prime}\right)$. By Grauert-Riemenschneider vanishing theorem $h^{1}\left(\left.Z\right|_{I}, \mathcal{L}\left(-\left.Z\right|_{\mathcal{V} \backslash I}\right)\right)=0$, hence $h^{1}(Z, \mathcal{L})=h^{1}\left(\left.Z\right|_{\mathcal{V} \backslash I}, \mathcal{L}\right)$. If $\mathcal{L}$ is associated with certain effective divisor $D \in$ $\mathrm{ECa}^{n l^{\prime}}(Z)$ (as the image of $c^{n l^{\prime}}$ ), then $\left.\mathcal{L}\right|_{\left.Z\right|_{\mathcal{V} \backslash I}}$ is associated with the restriction of this divisor to $\left.Z\right|_{\mathcal{V} \backslash I}$. But this restriction has an empty support, hence $\left.\mathcal{L}\right|_{\left.Z\right|_{\mathcal{V} \backslash I}}$ is the trivial bundle over $\left.Z\right|_{\mathcal{V} \backslash I}$.

(d) Since the restriction of any element of $\mathrm{ECa}^{n l^{\prime}}(Z)$ to $\left.Z\right|_{\mathcal{V} \backslash I}$ is the empty divisor, the image of the composition $\mathrm{ECa}^{n l^{\prime}}(Z) \rightarrow \mathrm{ECa}^{0}\left(\left.Z\right|_{\mathcal{V} \backslash I}\right) \rightarrow \operatorname{Pic}^{0}\left(\left.Z\right|_{\mathcal{V} \backslash I}\right)$ is the trivial bundle (that is, the zero element of $\operatorname{Pic}^{0}\left(Z_{\mathcal{V} \backslash I}\right)$ ). Therefore, $\operatorname{im}\left(c^{n l^{\prime}}\right) \subset$ $\operatorname{ker}\left(H^{1}\left(\mathcal{O}_{Z}\right) \rightarrow H^{1}\left(\mathcal{O}_{Z \mid \mathcal{V} \backslash I}\right)\right)$. Since they have the same dimension (cf. 6.1.12) they must agree.

(e) Let $n$ be so large that $\operatorname{im}\left(\widetilde{c}^{n l^{\prime}}\right)=A_{Z}\left(n l^{\prime}\right)$ is an affine subspace. We claim that any $\mathcal{L} \in \operatorname{im}\left(\widetilde{c}^{2 n l^{\prime}}\right)=A_{Z}\left(2 n l^{\prime}\right)$ is generated by global sections. Indeed, fix such a bundle and one of its sections $s \in H^{0}(Z, \mathcal{L})$ whose divisor is an element of $\mathrm{ECa}^{2 n l^{\prime}}(Z)$, whose support with reduced structure is $\mathbf{p}:=\left\{p_{1}, \ldots, p_{k}\right\} \subset E$. Let $\operatorname{ECa}_{p}^{n l^{\prime}}(Z)$ be the subspace of $\mathrm{ECa}^{n l^{\prime}}(Z)$ consisting of divisors supported in the complement of $\mathbf{p}$. This is a Zariski open set of $\mathrm{ECa}^{n l^{\prime}}(Z)$, hence $c\left(\mathrm{ECa}_{p}^{n l^{\prime}}(Z)\right)$ contains a Zariski open set $U$ in $A_{Z}\left(n l^{\prime}\right)$. Then $U+U=A_{Z}\left(2 n l^{\prime}\right)$, hence $\mathcal{L}$ admits a section whose divisor has support off $\mathbf{p}$. 


\subsection{Cohomological reinterpretations of $V_{Z}\left(I^{\prime}\right)$}

Fix $\mathcal{L} \in \operatorname{im}\left(c^{n l^{\prime}}\right)(n \gg 1), D \in\left(c^{n l^{\prime}}\right)^{-1}(\mathcal{L})$, and $s \in H^{0}(Z, \mathcal{L})$ without fixed components. Then, as in the situation of 3.2 one has the cohomological long exact sequence $H^{0}(Z, \mathcal{L}) \stackrel{R_{\mathcal{L}}}{\longrightarrow} \mathcal{O}_{D} \stackrel{\delta}{\longrightarrow} H^{1}\left(\mathcal{O}_{Z}\right) \rightarrow H^{1}(Z, \mathcal{L}) \rightarrow 0$ from (3.2.1). Then by Theorem 6.1.9, $\operatorname{im}\left(c^{n l^{\prime}}\right)=A\left(n l^{\prime}\right)$. Therefore, $\operatorname{im}\left(T_{D} c^{n l^{\prime}}\right) \subset T_{\mathcal{L}} A\left(n l^{\prime}\right)$. But, by Lemma 3.1.7, $\operatorname{dimim} T_{D} c^{n l^{\prime}}=\operatorname{dim} \mathrm{ECa}^{n l^{\prime}}(Z)-\operatorname{dimim}\left(c^{n l^{\prime}}\right)^{-1}(\mathcal{L})=$ $h^{1}\left(\mathcal{O}_{Z}\right)-h^{1}(Z, \mathcal{L})=\operatorname{dim} T_{\mathcal{L}} A\left(n l^{\prime}\right)=\operatorname{dim} V_{Z}\left(l^{\prime}\right)$. Hence, $\operatorname{im}\left(T_{D} \widetilde{c}^{n l^{\prime}}\right)=V_{Z}\left(l^{\prime}\right)$. As $\operatorname{im}\left(T_{D} \widetilde{c}^{n l^{\prime}}\right)=\operatorname{im} \delta$ (cf. Prop. 3.2.2) for $V_{Z}\left(l^{\prime}\right)$ we get two other cohomological reinterpretations. Either it is the Artin algebra $\mathcal{O}_{D} / \operatorname{im}\left(R_{\mathcal{L}}\right)$, as a vector space, identified as the image of $O_{D}$ into $H^{1}\left(\mathcal{O}_{Z}\right)$, or it is also the kernel of $H^{1}(\times s): H^{1}\left(\mathcal{O}_{Z}\right) \rightarrow H^{1}(Z, \mathcal{L})$.

In other words, for $n \gg 1$, the image of $\mathcal{O}_{D} \rightarrow H^{1}\left(\mathcal{O}_{Z}\right)$ is independent of the choice of $D$, while the kernel of $H^{1}(\times s): H^{1}\left(\mathcal{O}_{Z}\right) \rightarrow H^{1}(Z, \mathcal{L})$ is independent of the choice of $s$. Furthermore, they are equal, and in fact this subspace of $H^{1}\left(\mathcal{O}_{Z}\right)$ depends only on the $E^{*}$-support $I$ of $l^{\prime}$, and it equals $V_{Z}(I)$.

There is a parallel analogous discussion for $\widetilde{X}$ (instead of $Z$ ) as well (in that case the reduced structure of $D$ is Stein, hence $h^{1}\left(\mathcal{O}_{D}\right)=0$ again).

\subsection{Example. Characterization of the cases $\operatorname{dim} \operatorname{im}(c)=0$}

Fix $l^{\prime} \in-\mathcal{S}^{\prime}$ with $E^{*}$-support $I \subset \mathcal{V}$ and $Z>0$ as above. Using (3.1.8) and (6.1.12) one proves that the following facts are equivalent (for an additional equivalent property see also Example 8.1.4):

(i) $\operatorname{im}\left(c^{l^{\prime}}\right)$ is a point (or, $\left.V_{Z}\left(l^{\prime}\right)=0\right)$;

(ii) there exists $\mathcal{L} \in \operatorname{Pic}^{l^{\prime}}(Z)$ without fixed components such that $h^{1}(Z, \mathcal{L})=h^{1}(Z)$;

(iii) any $\mathcal{L} \in \operatorname{Pic}^{l^{\prime}}(Z)$ without fixed components satisfies $h^{1}(Z, \mathcal{L})=h^{1}(Z)$;

(iv) all line bundles $\mathcal{L} \in \mathrm{Pic}^{l^{\prime}}(Z)$ without fixed components are isomorphic to each other;

(v) $h^{1}\left(\mathcal{O}_{Z}\right)=h^{1}\left(\mathcal{O}_{Z \mid \mathcal{V} \backslash I}\right)$.

Let us define $\mathcal{S}_{p t}^{\prime}$ as $\left\{-l^{\prime} \in \mathcal{S}^{\prime}: \operatorname{im}\left(c^{l^{\prime}}\right)\right.$ is a point $\} \subset \mathcal{S}^{\prime}$, this is the set of Chern classes satisfying the above equivalent conditions. Using (6.1.2) we obtain that $\mathcal{S}_{p t}^{\prime}$ is a semigroup.

Part (v) via Proposition 5.5.1 reads as follows:

$$
\mathcal{S}_{p t}^{\prime}=\mathbb{Z}_{\geq 0}\left\langle E_{v}^{*}\left|E_{v} \not \subset\right| Z_{c o h}\left(Z, \mathcal{O}_{Z}\right) \mid\right\rangle
$$

Note that (in contrast with $\mathcal{S}_{d o m}^{\prime}$ ) $\mathcal{S}_{p t}^{\prime}$ is not topological. Indeed, take e.g. the graph from Example 3.4.1, $-l^{\prime}:=Z_{\min }=E_{v}^{*}$ (where $v$ is the (-2)-vertex adjacent with the (-7) vertex), and set $Z=Z_{K}$. Then, if $p_{g}(X, o)=2$ (that is, $(X, o)$ is Gorenstein) then $Z_{c o h}\left(Z, \mathcal{O}_{Z}\right)=Z$, and $\mathcal{S}_{p t}^{\prime}=\{0\}$. If $p_{g}(X, o)=1$, then $Z_{c o h}\left(Z, \mathcal{O}_{Z}\right)$ is the minimally elliptic cycle, and $\mathcal{S}_{p t}^{\prime}=\mathbb{Z}\left\langle E_{v}^{*}\right\rangle$.

In [51-53] a cycle $l \in \mathcal{S}^{\prime} \cap L$ is called $p_{g}$-cycle if $\mathcal{O}_{\widetilde{X}}(-l) \in \operatorname{Pic}(\tilde{X})$ has no fixed components, and $h^{1}\left(\widetilde{X}, \mathcal{O}_{\tilde{X}}(-l)\right)=p_{g}$. Note that this in our language means that $-l \in \mathcal{S}_{p t}^{\prime}$ for $Z \gg 0$. Our results generalizes several statements of [loc.cit.] 
for arbitrary bundles $\mathcal{L}$ without fixed components (replacing $\mathcal{O}_{\widetilde{X}}(-l)$ ) and arbitrary $\operatorname{dim} \operatorname{im}\left(c^{l^{\prime}}\right)$.

This particular case and several similar classical results valid for bundles of type $\mathcal{O}\left(l^{\prime}\right)$ motivate to investigate the position of the natural line bundles with respect to $\operatorname{im}\left(c^{l^{\prime}}\right)$ (i.e., whether $\mathcal{O}\left(l^{\prime}\right)$ has fixed components or no). This is the subject of Sect. 9.

\section{The Abel map via differential forms}

\subsection{Review of Laufer Duality [25], [26, p. 1281]}

Following Laufer, we identify the dual space $H^{1}\left(\widetilde{X}, \mathcal{O}_{\tilde{X}}\right)^{*}$ with the space of global holomorphic 2-forms on $\widetilde{X} \backslash E$ up to the subspace of those forms which can be extended holomorphically over $\widetilde{X}$.

For this, use first Serre duality $H^{1}\left(\widetilde{X}, \mathcal{O}_{\tilde{X}}\right)^{*} \simeq H_{c}^{1}\left(\widetilde{X}, \Omega_{\widetilde{X}}^{2}\right)$. Then, in the exact sequence

$0 \rightarrow H_{c}^{0}\left(\widetilde{X}, \Omega_{\widetilde{X}}^{2}\right) \rightarrow H^{0}\left(\widetilde{X}, \Omega_{\widetilde{X}}^{2}\right) \rightarrow H^{0}\left(\widetilde{X} \backslash E, \Omega_{\widetilde{X}}^{2}\right) \rightarrow H_{c}^{1}\left(\widetilde{X}, \Omega_{\widetilde{X}}^{2}\right) \rightarrow H^{1}\left(\widetilde{X}, \Omega_{\widetilde{X}}^{2}\right)$

$H_{c}^{0}\left(\widetilde{X}, \Omega_{\widetilde{X}}^{2}\right)=H^{2}\left(\widetilde{X}, \mathcal{O}_{\widetilde{X}}\right)^{*}=0$ by dimension argument, while $H^{1}\left(\widetilde{X}, \Omega_{\widetilde{X}}^{2}\right)=0$ by the Grauert-Riemenschneider vanishing. Hence,

$$
H^{1}\left(\widetilde{X}, \mathcal{O}_{\widetilde{X}}\right)^{*} \simeq H_{c}^{1}\left(\widetilde{X}, \Omega_{\widetilde{X}}^{2}\right) \simeq H^{0}\left(\widetilde{X} \backslash E, \Omega_{\widetilde{X}}^{2}\right) / H^{0}\left(\widetilde{X}, \Omega_{\widetilde{X}}^{2}\right) .
$$

The second isomorphism can be realized as follows. Fix a small tubular neighbourhood $N \subset \widetilde{X}$ of $E$ such that its closure is compact in $\widetilde{X}$. Take any $\omega \in H^{0}\left(\widetilde{X} \backslash E, \Omega_{\widetilde{X}}^{2}\right)$, and extend the restriction $\left.\omega\right|_{\tilde{X} \backslash N}$ to a $C^{\infty}(2,0)$-form $\tilde{\omega}$ on $\tilde{X}$. Then $\bar{\partial} \tilde{\omega}$ is a compactly supported $C^{\infty}(2,1)$-form, $\bar{\partial} \bar{\partial} \tilde{\omega}=0$, hence $\bar{\partial} \tilde{\omega}$ determines a class in $H_{c}^{1}\left(\widetilde{X}, \Omega^{2}\right)$. If $\tilde{\omega}$ is a holomorphic extension then $\bar{\partial} \tilde{\omega}=0$. Next, let $\lambda$ be a $C^{\infty}(0,1)$ form in $\widetilde{X}$. Then the duality $H^{1}\left(\widetilde{X}, \mathcal{O}_{\widetilde{X}}\right) \otimes H_{c}^{1}\left(\widetilde{X}, \Omega^{2}\right) \rightarrow \mathbb{C}$ is the perfect pairing

$$
\langle[\lambda],[\bar{\partial} \tilde{\omega}]\rangle=\int_{\widetilde{X}} \lambda \wedge \bar{\partial} \tilde{\omega} .
$$

Assume that the class $[\lambda] \in H^{1}\left(\tilde{X}, \mathcal{O}_{\tilde{X}}\right)$ is realized by a $\check{C}$ ech cocyle $\lambda_{i j} \in \mathcal{O}\left(U_{i} \cap U_{j}\right)$, where $\left\{U_{i}\right\}_{i}$ is an open cover of $E, U_{i} \cap U_{j} \cap U_{k}=\emptyset$, and each connected component of the intersections $U_{i} \cap U_{j}$ is either a coordinate bidisc $B=\{|u|<2 \epsilon,|v|<2 \epsilon\}$ with coordinates $(u, v)$, such that $E \cap B \subset\{u v=0\}$, or a punctured coordinate bidisc $B=\{\epsilon / 2<|v|<2 \epsilon,|u|<2 \epsilon\}$ with coordinates $(u, v)$, such that $E \cap B=\{u=0\}$. Then $\lambda$ is obtained as follows: one finds $C^{\infty}$ functions $\lambda_{i}$ on $U_{i}$ such that $\lambda_{i}-\lambda_{j}=\lambda_{i j}$ on $U_{i} \cap U_{j}$, and one sets $\lambda$ as $\bar{\partial} \lambda_{i}$ on $U_{i}$. Then, by Stokes theorem

$$
\langle[\lambda],[\bar{\partial} \tilde{\omega}]\rangle=\sum_{B} \int_{|u|=\epsilon,|v|=\epsilon} \lambda_{i j} \omega .
$$


By Stokes theorem, if $\omega$ has no pole along $E$ in $B$, then the $B$-contribution in the above sum is zero.

7.1.3 Above $H^{0}\left(\tilde{X} \backslash E, \Omega_{\widetilde{X}}^{2}\right)$ can be replaced by $H^{0}\left(\widetilde{X}, \Omega_{\widetilde{X}}^{2}(Z)\right)$ for a large cycle $Z$ (e.g. for $Z \geq\left\lfloor Z_{K}\right\rfloor$ ). Indeed, for any cycle $Z>0$ from the exacts sequence of sheaves $0 \rightarrow \Omega_{\widetilde{X}}^{2} \rightarrow \Omega_{\widetilde{X}}^{2}(Z) \rightarrow \mathcal{O}_{Z}\left(Z+K_{\widetilde{X}}\right) \rightarrow 0$ and from the vanishing $h^{1}\left(\Omega_{\widetilde{X}}^{2}\right)=0$ and Serre duality one has

$$
H^{0}\left(\Omega_{\widetilde{X}}^{2}(Z)\right) / H^{0}\left(\Omega_{\widetilde{X}}^{2}\right)=H^{0}\left(\mathcal{O}_{Z}(Z+K)\right) \simeq H^{1}\left(\mathcal{O}_{Z}\right)^{*}
$$

Since $H^{1}\left(\mathcal{O}_{Z}\right) \simeq H^{1}\left(\mathcal{O}_{\tilde{X}}\right)$ for $Z \geq\left\lfloor Z_{K}\right\rfloor$, the natural inclusion

$$
H^{0}\left(\Omega_{\widetilde{X}}^{2}(Z)\right) / H^{0}\left(\Omega_{\widetilde{X}}^{2}\right) \hookrightarrow H^{0}\left(\widetilde{X} \backslash E, \Omega_{\widetilde{X}}^{2}\right) / H^{0}\left(\Omega_{\widetilde{X}}^{2}\right)
$$

is an isomorphism.

7.1.6 The above duality, via the isomorphism exp $: H^{1}\left(\widetilde{X}, \mathcal{O}_{\tilde{X}}\right) \rightarrow c^{-1}(0) \subset$ $H^{1}\left(\widetilde{X}, \mathcal{O}_{\widetilde{X}}^{*}\right)=\operatorname{Pic}(\widetilde{X})$, can be transported as follows. Consider the following situation. We fix a smooth point $p$ on $E$, a local bidisc $B \ni p$ with local coordinates $(u, v)$ such that $B \cap E=\{u=0\}$. We assume that a certain form $\omega \in H^{0}\left(\widetilde{X}, \Omega_{\widetilde{X}}^{2}(Z)\right)$ has local equation $\omega=\sum_{i \in \mathbb{Z}, j \geq 0} a_{i, j} u^{i} v^{j} d u \wedge d v$ in $B$.

In the same time, we fix a divisor $\widetilde{D}$ on $\widetilde{X}$, whose local equation in $B$ is $v^{n}, n \geq 1$. Let $\widetilde{D}_{t}$ be another divisor, which is the same as $\widetilde{D}$ in the complement of $B$ and in $B$ its local equation is $\left(v+t u^{o-1}\right)^{n}$, where $o \geq 1$ and $t \in \mathbb{C}($ with $|t| \ll 1$ whenever $o=1)$.

Next we will provide three type of formulae.

The first one is the composition of several maps. Note that the pairing $\langle\cdot,[\bar{\partial} \tilde{\omega}]\rangle$ (abridged as $\langle\cdot, \omega\rangle)$ produces a map $H^{1}\left(\widetilde{X}, \mathcal{O}_{\tilde{X}}\right) \rightarrow \mathbb{C}$. Then we identify $H^{1}\left(\widetilde{\widetilde{X}}, \mathcal{O}_{\tilde{X}}\right)$ with $\operatorname{Pic}^{0}(\widetilde{X})$ by the exponential map. Then we consider the composition $t \mapsto \widetilde{D}_{t}-$ $\widetilde{D} \mapsto \mathcal{O}_{\widetilde{X}}\left(\widetilde{D}_{t}-\widetilde{D}\right) \mapsto \exp ^{-1} \mathcal{O}_{\widetilde{X}}\left(\widetilde{D}_{t}-\widetilde{D}\right) \mapsto\left\langle\exp ^{-1} \mathcal{O}_{\widetilde{X}}\left(\widetilde{D}_{t}-\widetilde{D}\right), \omega\right\rangle$. The first formula makes this composition explicit. This restricted to any cycle $Z \gg 0$ can be reinterpreted as $\omega$-coordinate of the Abel map restricted to the path $t \mapsto D_{t}:=\left.\widetilde{D}_{t}\right|_{Z}$ (and shifted by the image of $D:=\left.\widetilde{D}\right|_{Z}$ ).

The second formula determines the tangent application of the above composition (in this way it determines the $\omega$-coordinate of the tangent application of the Abel map restricted to $D_{t}$ ).

In the third formula we replace the path $D_{t}$ by a complete neighborhood of $D$ in $\mathrm{ECa}(Z)$.

Note that if we consider-instead of a single form $\omega-$ a complete set of representatives of a basis of $H^{0}\left(\widetilde{X}, \Omega_{\widetilde{X}}^{2}(Z)\right) / H^{0}\left(\widetilde{X}, \Omega_{\widetilde{X}}^{2}\right)$, then we get by the above three constructions the restriction of the Abel map to the path $D_{t}$, the tangent map of this restriction, and in the third case the 'complete' Abel map defined in some neighbourhood of $D$. 


\subsection{The Abel map restricted to $D_{t}$}

The first two cases start with the explicit computation of $\left\langle\exp ^{-1} \mathcal{O}_{\widetilde{X}}\left(\widetilde{D}_{t}-\widetilde{D}\right), \omega\right\rangle$, as follows. $\widetilde{D}_{t}-\widetilde{D}$ is the divisor $\widetilde{D}^{\prime}=\operatorname{div}\left(\left(v+t u^{o-1}\right) / v\right)^{n}$, supported in $B=$ $\{|u|,|v|<\epsilon\}$. We can fix $\epsilon$ such that the support of $\widetilde{D}^{\prime}$ is in $\{|v|<\epsilon / 2\}$, and set $B^{*}:=\{\epsilon / 2<|v|<\epsilon,|u|<\epsilon\}$. Using the trivialization of $\mathcal{O}\left(\widetilde{D}^{\prime}\right)$ in $\widetilde{X} \backslash\{|v| \leq \epsilon / 2\}$ and the realization $\mathcal{O}\left(\widetilde{D}^{\prime}\right)$ on $B$, we get that $\mathcal{O}\left(\widetilde{D}^{\prime}\right)$ can be represented by the cocycle $g=\left(\left(v+t u^{o-1}\right) / v\right)^{n} \in \mathcal{O}^{*}\left(B^{*}\right)$. Therefore, $\log \left(\left(v+t u^{o-1}\right) / v\right)^{n}=n \log (1+$ $\left.t u^{o-1} / v\right)$ is a cocycle in $B^{*}$ representing its lifting into $H^{1}\left(\widetilde{X}, \mathcal{O}_{\widetilde{X}}\right)$. This paired with $\omega$ gives:

$$
\begin{aligned}
& \left\langle\left\langle\widetilde{D}_{t}, \omega\right\rangle\right\rangle:=\left\langle\exp ^{-1} \mathcal{O}_{\widetilde{X}}\left(\widetilde{D}_{t}-\widetilde{D}\right), \omega\right\rangle \\
& \quad=n \int_{|u|=\epsilon,|v|=\epsilon} \log \left(1+t \frac{u^{o-1}}{v}\right) \cdot \sum_{i \in \mathbb{Z}, j \geq 0} a_{i, j} u^{i} v^{j} d u \wedge d v .
\end{aligned}
$$

If $\omega_{1}, \ldots, \omega_{p_{g}}$ are representatives of a basis for $H^{0}\left(\widetilde{X}, \Omega_{\widetilde{X}}^{2}(Z)\right) / H^{0}\left(\widetilde{X}, \Omega_{\widetilde{X}}^{2}\right)$, and $Z \gg 0$, then

$$
\widetilde{D}_{t} \mapsto\left(\left\langle\left\langle\widetilde{D}_{t}, \omega_{1}\right\rangle\right\rangle, \ldots,\left\langle\left\langle\widetilde{D}_{t}, \omega_{p_{g}}\right\rangle\right\rangle\right)
$$

is the restriction of the Abel map to $\widetilde{D}_{t}$ (associated with $Z$, and shifter by the image of $\widetilde{D})$.

At the level of tangent application on has the formula for $\left.\left(T_{\widetilde{c}(D)} \omega\right) \circ T_{D} \widetilde{c}\right)\left(\left.\frac{d}{d t} D_{t}\right|_{t=0}\right)$ :

$$
\begin{aligned}
& \left.\frac{d}{d t}\right|_{t=0}\left[n \int_{|u|=\epsilon,|v|=\epsilon} \log \left(1+t \frac{u^{o-1}}{v}\right) \cdot \sum_{i \in \mathbb{Z}, j \geq 0} a_{i, j} u^{i} v^{j} d u \wedge d v\right] \\
& =\lambda \cdot a_{-o, 0}\left(\lambda \in \mathbb{C}^{*}\right) .
\end{aligned}
$$

If $\omega$ has no pole along the divisor $\{u=0\}$ then $\left\langle\exp ^{-1} \mathcal{O}_{\widetilde{X}}\left(\widetilde{D}_{t}-\widetilde{D}\right), \omega\right\rangle=0$ for any path $\widetilde{D}_{t}$.

Definition 7.2.4 Consider the above situation in the bidisc $B: B \cap E=\{u=0\}, \widetilde{D}$ has local equation $v$ (i.e. $n=1$ ), and $\omega=\sum_{i \in \mathbb{Z}, j \geq 0} a_{i, j} u^{i} v^{j} d u \wedge d v$. Then we introduce the Leray residue of $\omega / d u$ along $\{v=0\}$ as the 1 -form (with possible poles at $\widetilde{D} \cap E$ ) defined by $\left.(\omega / d v)\right|_{v=0}=\sum_{i} a_{i, 0} u^{i} d u$. We denote it by $\operatorname{Res}_{D}(\omega)$.

Note that the right hand side of (7.2.3) tests exactly the pole part of the Leray residue $\operatorname{Res}_{D}(\omega)$.

\subsection{The Abel map}

Assume as above that in the ball $B$ the divisor $\widetilde{D}$ is given by $v=0$ (i.e. $n=1$ ), and its 'perturbation' $\widetilde{D}(c)$ is given by $v=c_{0}+c_{1} u+c_{2} u^{2}+\cdots$ with $\left|c_{0}\right| \ll \epsilon$. Furthermore, 
assume that the form $\omega$ in $B$ has the form $\left(f(v) / u^{\ell+1}\right) d u \wedge d v$, where $f \in \mathcal{O}(B)$ and $\ell \geq 0$. (Note that the Laurent expansion in variable $u$ of any differential form is a sum of such terms.)

Our aim is the computation of $\langle\langle\widetilde{D}(c), \omega\rangle\rangle$.

If $\left\{p_{i}\right\}_{\geq 1}$ (resp. $\left\{h_{i}\right\}_{i \geq 1}$ ) denote the power sum (resp. complete) symmetric polynomials (functions) then (cf. [30, p. 23])

$$
p_{1} u+p_{2} u^{2} / 2+p_{3} u^{3} / 3+\cdots=\log \left(1+h_{1} u+h_{2} u^{2}+\cdots\right) .
$$

Furthermore, by [30, p. 28], for $n \geq 1$,

$$
(-1)^{n+1} p_{n}=\left(\begin{array}{ccccc}
h_{1} & 1 & 0 & \ldots & 0 \\
2 h_{2} & h_{1} & 1 & \ldots & 0 \\
\vdots & \vdots & \vdots & & \vdots \\
n h_{n} & h_{n-1} & h_{n-2} & \ldots & h_{1}
\end{array}\right)
$$

We rewrite (7.3.1) as $\log (A)+p_{1} u+p_{2} u^{2} / 2+\cdots=\log \left(A+h_{1} A u+h_{2} A u^{2}+\cdots\right)$ and we make the substitution $A=\left(v-c_{0}\right) / v, h_{1} A=-c_{1} / v, h_{2} A=-c_{2} / v$, etc., and we obtain

$$
\log \left(1-\frac{c_{0}+c_{1} u+c_{2} u^{2}+\cdots}{v}\right)=\log \left(1-\frac{c_{0}}{v}\right)+\delta_{1}(c) u+\delta_{2}(c) u^{2}+\cdots,
$$

where for $n \geq 1$

$$
\delta_{n}(c)=\sum_{i=1}^{n} \frac{\delta_{n, i}(c)}{\left(v-c_{0}\right)^{i}}=\frac{-1}{n}\left(\begin{array}{ccccc}
\frac{c_{1}}{v-c_{0}} & -1 & 0 & \ldots & 0 \\
\frac{2 c_{2}}{v-c_{0}} & \frac{c_{1}}{v-c_{0}} & -1 & \ldots & 0 \\
\vdots & \vdots & \vdots & & \vdots \\
\frac{n c_{n}}{v-c_{0}} & \frac{c_{n-1}}{v-c_{0}} & \frac{c_{n-2}}{v-c_{0}} & \cdots & \frac{c_{1}}{v-c_{0}}
\end{array}\right) .
$$

Note that $\delta_{n, i}$ are certain universal polynomials in variables $c_{1}, \ldots, c_{n}$. Then $\langle\langle\widetilde{D}(c), \omega\rangle\rangle$ equals

$$
\int_{|u|=\epsilon,|v|=\epsilon} \log \left(1-\frac{c_{0}+c_{1} u+\cdots}{v}\right) \cdot \frac{f(v)}{u^{\ell+1}} d u \wedge d v=\sum_{i=1}^{\ell} \frac{\delta_{\ell, i}(c)}{(i-1) !} \cdot \frac{d^{i-1} f}{d v^{i-1}}\left(c_{0}\right) .
$$

\subsection{Reduction to an arbitrary $Z>0$}

Consider the above perfect pairing $H^{1}\left(\widetilde{X}, \mathcal{O}_{\tilde{X}}\right) \otimes H^{0}\left(\tilde{X} \backslash E, \Omega_{\widetilde{X}}^{2}\right) / H^{0}\left(\Omega_{\widetilde{X}}^{2}\right) \rightarrow \mathbb{C}$ given via integration of class representatives. In $H^{1}\left(\widetilde{X}, \mathcal{O}_{\tilde{X}}\right)$ let $A$ be the image of the $H^{1}\left(\widetilde{X}, \mathcal{O}_{\widetilde{X}}(-Z)\right)$, hence $H^{1}\left(\widetilde{X}, \mathcal{O}_{\widetilde{X}}\right) / A=H^{1}\left(\mathcal{O}_{Z}\right)$. On the other hand, 
in $H^{0}\left(\widetilde{X} \backslash E, \Omega_{\widetilde{X}}^{2}\right) / H^{0}\left(\Omega_{\widetilde{X}}^{2}\right)$ consider the subspace $B:=H^{0}\left(\Omega_{\widetilde{X}}^{2}(Z)\right) / H^{0}\left(\Omega_{\widetilde{X}}^{2}\right)$ of dimension $h^{1}\left(\mathcal{O}_{Z}\right)$ (cf. (7.1.4). Since $\langle A, B\rangle=0$, the pairing factorizes to a perfect pairing $H^{1}\left(\mathcal{O}_{Z}\right) \otimes H^{0}\left(\Omega_{\widetilde{X}}^{2}(Z)\right) / H^{0}\left(\Omega_{\widetilde{X}}^{2}\right) \rightarrow \mathbb{C}$. It can be described by the very same integral form of the corresponding class representatives.

Moreover, if $\widetilde{D}_{t}$ is an 1-parameter family of divisors as in 7.1.6, representing an element in $H^{1}\left(\mathcal{O}_{Z}\right)$ (via the surjection $H^{1}\left(\mathcal{O}_{\widetilde{X}}\right) \rightarrow H^{1}\left(\mathcal{O}_{Z}\right)$ ), and $\omega$ is a representative of a class $[\omega] \in H^{0}\left(\Omega_{\widetilde{X}}^{2}(Z)\right) / H^{0}\left(\Omega_{\widetilde{X}}^{2}\right)$, then the expression of the pairing $H^{1}\left(\mathcal{O}_{Z}\right) \otimes$ $H^{0}\left(\Omega_{\widetilde{X}}^{2}(Z)\right) / H^{0}\left(\Omega_{\widetilde{X}}^{2}\right) \rightarrow \mathbb{C},\left\langle\exp ^{-1} \mathcal{O}_{Z}\left(\widetilde{D}_{t}-\widetilde{D}\right),[\omega]\right\rangle$, can be represented by the very same formula (7.2.1) (as in the case $Z \gg 0$ ). Furthermore, all other formulae of Sects. 7.2 and 7.3 also have their extended versions. E.g., (7.2.3) gives $T_{\widetilde{c}(D)}(\omega) \circ$ $\left.T_{D} \widetilde{c}^{\prime}(Z)\right)\left(\left.\frac{d}{d t} D_{t}\right|_{t=0}\right)$, and (7.3.5) is the $[\omega]$-coordinate of the Abel map ECa ${ }^{\prime}(Z) \rightarrow$ $H^{1}\left(\mathcal{O}_{Z}\right)$.

\section{The 'stable' arrangement $\left\{V_{\widetilde{X}}(I)\right\}_{I C \mathcal{V}}$ and differential forms}

\subsection{The arrangement $\left\{\Omega_{\tilde{X}}(I)\right\}_{\text {I }}$ of forms and its duality with $\left\{V_{\widetilde{X}}(I)\right\}_{\text {I }}$}

Definition 8.1.1 Let $\Omega_{\widetilde{X}}(I)$ (or, $\left.\Omega(I)\right)$ be the subspace of $H^{0}\left(\widetilde{X} \backslash E, \Omega_{\widetilde{X}}^{2}\right) / H^{0}\left(\widetilde{X}, \Omega_{\widetilde{X}}^{2}\right)$ generated by differential forms $\omega \in H^{0}\left(\widetilde{X} \backslash E, \Omega_{\widetilde{X}}^{2}\right)$, which have no poles along $E_{I} \backslash \cup_{v \notin I} E_{v}$.

As in Theorem 6.1.9(c), let $\left(\tilde{X} / E_{\mathcal{V} \backslash I}, o \mathcal{V} \backslash I\right)$ denote the multi-germ obtained by contracting the connected components of $E_{\mathcal{V} \backslash I}$ in $\tilde{X}$. Let $\tilde{X}(\mathcal{V} \backslash I)$ be a small neighbourhood of $E_{\mathcal{V} \backslash I}$ in $\tilde{X}$, which is the inverse image by $\phi$ of a small Stein neighbourhood of $\left(\tilde{X} / E_{\mathcal{V} \backslash I}, o \mathcal{V} \backslash I\right)$.

Proposition 8.1.2 (a) $\operatorname{dim} \Omega(I)=p_{g}\left(\tilde{X} / E_{\mathcal{V} \backslash I}, o \mathcal{V} \backslash I\right)$.

(b) Set $\bar{\Omega}(\emptyset):=H^{0}\left(\widetilde{X}(\mathcal{V} \backslash I) \backslash E_{\mathcal{V} \backslash I}, \Omega_{\widetilde{X}(\mathcal{V} \backslash I)}^{2}\right) / H^{0}\left(\widetilde{X}(\mathcal{V} \backslash I), \Omega_{\widetilde{X}(\mathcal{V} \backslash I)}^{2}\right)$. Then linear map $\rho: \Omega(I) \rightarrow \bar{\Omega}(\emptyset)$, induced by restriction, is an isomorphism.

(c) Fix $I \subset \mathcal{V}$ as above and set $J \subset \mathcal{V}$ with $J \cap I=\emptyset$. Let $\bar{\Omega}(J)$ be the subspace of $\bar{\Omega}(\emptyset)$ generated by forms from $H^{0}\left(\widetilde{X}(\mathcal{V} \backslash I) \backslash E_{\mathcal{V} \backslash I}, \Omega_{\widetilde{X}}^{2}(\mathcal{V} \backslash I)\right.$ without pole along $E_{J}$. Then the restriction of $\rho$ to $\Omega(J) \cap \Omega(I)$ induces an isomorphism $\Omega(J) \cap$ $\Omega(I) \rightarrow \bar{\Omega}(J)$.

In particular, for any $I$, the subspace arrangement $\{\bar{\Omega}(J)\}_{J \cap I=\emptyset}$ of the multi$\operatorname{germ}\left(\widetilde{X} / E_{\mathcal{V} \backslash I}, o_{\mathcal{V} \backslash I}\right)$ and resolution $\widetilde{X}(\mathcal{V} \backslash I)$ can be recovered from the arrangement $\{\Omega(M)\}_{M}$ via $\{\Omega(I) \cap \Omega(J)\}_{J \cap I=\emptyset \text {. }}$

Proof (a) Fix $Z=\sum_{v \in \mathcal{V} \backslash I} n_{v} E_{v}$ with all $n_{v} \gg 0$. By (7.1.4) $\operatorname{dim} \Omega(I)=$ $\operatorname{dim} H^{0}\left(\Omega_{\widetilde{X}}^{2}(Z)\right) / H^{0}\left(\Omega_{\widetilde{X}}^{2}\right)=h^{1}\left(\mathcal{O}_{Z}\right)$, which equals $p_{g}\left(\tilde{X} / E_{\mathcal{V} \backslash I}, o \mathcal{V} \backslash I\right)$ by formal function theorem.

(b) If $[\omega] \in \operatorname{ker}(\rho)$, then $\omega$ has no pole along $E_{I}$ (since $[\omega] \in \Omega(I)$ ), and has no pole along $E_{\mathcal{V} \backslash I}$ either (since $\rho[\omega]=0$ ). Hence $[\omega]=0$, and $\rho$ is injective. Since by (a) the dimension of the source and the target is the same, $\rho$ is an isomorphism. 
(c) By $(b)$, for any $\bar{\omega} \in \bar{\Omega}(J)$ there exists $\omega \in \Omega(I)$ with $\rho(\omega)=\bar{\omega}$. Note that $\omega$ is necessarily in $\Omega(I \cap J)$, hence $\Omega(J) \cap \Omega(I) \rightarrow \bar{\Omega}(J)$ is onto.

The next result shows that the linear subspace arrangement $\left\{V_{\widetilde{X}}(I)\right\}_{I}$ of $H^{1}\left(\widetilde{X}, \mathcal{O}_{\widetilde{X}}\right)$ (cf. $\underset{\widetilde{X}}{6.1 .7)}$ is dual to the linear subspace arrangement $\left\{\Omega_{\widetilde{X}}(I)\right\}_{I}$ of $\Omega_{\widetilde{X}}(\emptyset)=$ $H^{0}\left(\widetilde{X} \backslash E, \Omega_{\widetilde{X}}^{2}\right) / H^{0}\left(\widetilde{X}, \Omega_{\widetilde{X}}^{2}\right)$.

Theorem 8.1.3 Via duality (7.1.1) one has $V_{\widetilde{X}}(I)^{*}=\Omega_{\widetilde{X}}(I)$.

Proof We fix a cycle $Z \gg 0$ for which $V_{Z}(I)=V_{\widetilde{X}}(I)$. Choose $l^{\prime}=-\sum_{v \in I} a_{v} E_{v}^{*}$ such that each $a_{v}$ is so large that $\operatorname{im}\left(c^{l^{\prime}}\right)$ is an affine space, cf. Theorem 6.1.9. Then, any element $\mathcal{L}$ of $V_{Z}(I)$ has the form $\mathcal{O}_{Z}\left(D_{1}-D_{2}\right)$, with both $D_{1}, D_{2} \in \mathrm{ECa}^{l^{\prime}}(Z)$. Lift $\left\{D_{i}\right\}_{i=1,2}$ to effective divisors $\left\{D_{1}^{\prime}\right\}_{i=1,2}$ in $\widetilde{X}$. Since they do not intersect $E_{\mathcal{V} \backslash I}$, the class $[\lambda]$ of $\mathcal{O}_{\widetilde{X}}\left(D_{1}^{\prime}-D_{2}^{\prime}\right)$ in $\operatorname{Pic}^{0}(\widetilde{X})$ can be represented by a Eech cocycles $\left\{\lambda_{i j}\right\}$, which in a neighbourhood of $E_{\mathcal{V} \backslash I}$ are all zero. Therefore, if $\omega$ is a form which has no pole along $E_{I},\langle[\lambda],[\omega]\rangle=0$ by (7.1.2). That is, $\left\langle V_{\widetilde{X}}(I), \Omega(I)\right\rangle=0$, or $V_{\widetilde{X}}(I) \subset \Omega(I)^{*}$. Since by (6.1.12) and Proposition 8.1.2(a) one has $\operatorname{dim} V_{\widetilde{X}}(I)=$ $p_{g}-\operatorname{dim} \Omega(I)$, we get $V_{\widetilde{X}}(I)=\Omega(I)^{*}$.

Example 8.1.4 (Continuation of Example 6.3) Fix $l^{\prime} \in-\mathcal{S}^{\prime}$ with $E^{*}$-support $I \subset \mathcal{V}$ as in 6.3, and choose $Z \gg 0$. Then

$$
\operatorname{im}\left(c^{l^{\prime}}\right) \text { is a point } \Leftrightarrow V_{\widetilde{X}}(I)=0 \Leftrightarrow \Omega_{\widetilde{X}}(I)=\Omega_{\widetilde{X}}(\emptyset) .
$$

\subsection{Convexity property of $\Omega(\{v\})$ 's}

Clearly, the subspace arrangement has the properties $\Omega(\emptyset) \simeq \mathbb{C}^{p_{g}}$, and $\Omega(I \cup J)=$ $\Omega(I) \cap \Omega(J)$. In this subsection we establish an interesting additional structure property of the arrangement. It is the analytical analogue of topological convexity property [23, Prop. 4.4.1].

For simplicity write $\Omega_{v}:=\Omega(\{v\})$ for $v \in \mathcal{V}$, and define

$$
\Pi(I):= \begin{cases}\emptyset & \text { if } I=\emptyset \\ \sum_{v \in I} \Omega_{v} & \text { if } I \neq \emptyset .\end{cases}
$$

Proposition 8.2.1 For any $I \subset \mathcal{V}$ let $\Gamma_{I}$ be the smallest connected subtree of $\Gamma$ whose set of vertices $\bar{I}$ contains $I$. Then $\Pi(J)=\Pi(I)$ for any $I \subset J \subset \bar{I}$.

Proof By induction, it is enough to consider the case $J=I \cup\{u\}$, such that $u$ is on the geodesic path connecting $v, w$ with $v, w \in I$. Moreover, it is enough to show that $\Omega_{u} \subset \Omega_{v}+\Omega_{w}$. Write the connected components of $\Gamma \backslash u$ as $\cup_{k=0}^{s} \Gamma_{k}$, and set $I_{k}:=\mathcal{V}\left(\Gamma_{k}\right)$. Assume that $w \in I_{0}$.

Choose an arbitrary $\omega \in \Omega_{u}$ and consider its restriction $\left.\omega\right|_{\widetilde{X}\left(I_{0}\right)}$ in $\bar{\Omega}(\emptyset):=$ $H^{0}\left(\Omega^{2}\left(\tilde{X}\left(I_{0}\right) \backslash E_{I_{0}}\right)\right) / H^{0}\left(\Omega^{2}\left(\tilde{X}\left(I_{0}\right)\right)\right)$. By Proposition 8.1.2(b) $\Omega\left(\mathcal{V} \backslash I_{0}\right) \rightarrow \bar{\Omega}(\emptyset)$ is bijective, hence there exists $\omega_{v} \in \Omega\left(\mathcal{V} \backslash I_{0}\right)$ such that $\left.\omega_{v}\right|_{\widetilde{X}\left(I_{0}\right)}=\left.\omega\right|_{\tilde{X}\left(I_{0}\right)}$. But $\Omega_{v} \supset \Omega\left(\mathcal{V} \backslash I_{0}\right)$, hence $\omega_{v} \in \Omega_{v}$. On the other hand, $\left.\left(\omega-\omega_{v}\right)\right|_{\tilde{X}\left(I_{0}\right)}=0$, hence $\omega_{w}:=\omega-\omega_{v} \in \Omega_{w}$. Thus $\omega=\omega_{v}+\omega_{w} \in \Omega_{v}+\Omega_{w}$. 
Example 8.2.2 Consider the weighted homogeneous isolated hypersurface singularity $(X, o)=\left\{x^{4}+y^{4}+z^{5}=0\right\} \subset\left(\mathbb{C}^{3}, 0\right)$. One verifies that $p_{g}=4$ (use either [55], or Sect. 12 from here). We consider the minimal good resolution, whose graphs is

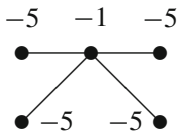

If $\omega$ is the Gorenstein form, then $\omega, z \omega, x \omega$ and $y \omega$ generate $H^{0}\left(\widetilde{X} \backslash E, \Omega_{\widetilde{X}}^{2}\right) / H^{0}$ $\left(\Omega_{\widetilde{X}}^{2}\right)$. The pole orders along the central curve $E_{0}$ are $7,3,2,2$. Let $v_{i}(1 \leq i \leq 4)$ be the end-vertices. Then for fixed $i, \mathcal{V} \backslash\left\{v_{i}\right\}$ represents a minimally elliptic singularity. Hence $\Omega_{v_{i}} \simeq \mathbb{C}$ by (6.1.12) and Theorem 8.1.3. If $\xi_{i}$ are the roots of $\xi^{4}+1=0$, then $\left(x+\xi_{i} y\right) \omega$ generates $\Omega_{v_{i}}$, hence $\sum_{i=1}^{4} \Omega_{v_{i}} \simeq \mathbb{C}^{2}=\langle x \omega, y \omega\rangle$.

In particular, the linear subspace arrangement $\left\{\Omega_{v}\right\}_{v}$ in $\mathbb{C}^{p_{g}}=\mathbb{C}^{4}$ is not generic at all. Furthermore, $\Omega_{v_{0}}=0$ hence 8.2 .1 can also be exemplified on this concrete example.

\subsection{Reduction to an arbitrary $Z>0$}

The duality from Theorem 8.1.3, valid for $\tilde{X}$ (or, for any $Z \gg 0$ ) can be generalized for any $Z \geq E$ as follows. For the definition of $V_{Z}(I)$ see Definitions 6.1.3 and 6.1.7. In parallel, define $\Omega_{Z}(I)$ as the subspace $H^{0}\left(\Omega_{\widetilde{X}}^{2}\left(\left.Z\right|_{\mathcal{V} \backslash I}\right)\right) / H^{0}\left(\Omega_{\widetilde{X}}^{2}\right)$ in $H^{0}\left(\Omega_{\widetilde{X}}^{2}(Z)\right) / H^{0}\left(\Omega_{\widetilde{X}}^{2}\right)$. By (7.1.4) $\operatorname{dim} H^{0}\left(\Omega_{\widetilde{X}}^{2}(Z)\right) / H^{0}\left(\Omega_{\widetilde{X}}^{2}\right)=h^{1}\left(\mathcal{O}_{Z}\right)$, while $\operatorname{dim} \Omega_{Z}(I)=h^{1}\left(\mathcal{O}_{Z \mid \mathcal{V} \backslash I}\right)$. But, by pairing (similarly as in the proof of Theorem 8.1.3) $V_{Z}(I) \subset \Omega_{Z}(I)^{*}$. Furthermore, by (6.1.12), $\operatorname{dim} V_{Z}(I)=\operatorname{dim} \Omega_{Z}(I)^{*}$. Hence

$$
V_{Z}(I)=\Omega_{Z}(I)^{*}
$$

\section{The 'stable' dimensions $\left\{\operatorname{dim}\left(V_{Z}(I)\right)\right\}_{I}$ and natural line bundles}

9.1. Recall that the saturation in $\mathcal{S}^{\prime}$ of a submonoid $\mathcal{M} \subset \mathcal{S}^{\prime}$ is the submonoid $\overline{\mathcal{M}}:=\left\{l^{\prime} \in \mathcal{S}^{\prime}: \exists n \geq 1\right.$ with $\left.n l^{\prime} \in \mathcal{M}\right\}$.

Let us fix some cycle $Z \geq E$. Recall that $\mathrm{ECa}^{-l^{\prime}}(Z) \neq \emptyset$ if and only if $l^{\prime} \in \mathcal{S}^{\prime}$. For $l^{\prime} \in \mathcal{S}^{\prime}$ regarding the mutual position of the natural line bundle $\mathcal{O}_{Z}\left(-l^{\prime}\right)$ with respect to the image of $c^{-l^{\prime}}: \mathrm{ECa}^{-l^{\prime}}(Z) \rightarrow \mathrm{Pic}^{-l^{\prime}}(Z)$ we can consider three cases.

(a) $\mathcal{O}_{Z}\left(-l^{\prime}\right) \in \operatorname{im}\left(c^{-l^{\prime}}\right)$, or, equivalently, $0 \in \operatorname{im}\left(\widetilde{c}^{-l^{\prime}}\right)$. The set of cycles $l^{\prime}$ satisfying this property is denoted by $\mathcal{S}_{i m}^{\prime}$. Clearly $0 \in \mathcal{S}_{i m}^{\prime}$ and by the first paragraphs of 6.1 it is a sub-monoids of $\mathcal{S}^{\prime}$. (In the literature, this monoid-defined for bundles over $Z \gg 0$, or over $\widetilde{X}$-is called the analytic monoid of $(X, o)$, in contrast with the topological monoid $\mathcal{S}^{\prime}$, since it indexes the restrictions to $E$ of the divisors of different holomorhic sections of the natural line bundles of $\widetilde{X}$, or divisors of functions of the universal abelian covering of $(X, o), \mathrm{cf}$. [34].)

(b) $\mathcal{O}_{Z}\left(-n l^{\prime}\right) \in \operatorname{im}\left(c^{-n l^{\prime}}\right)$, or $0 \in \operatorname{im}\left(\widetilde{c}^{-n l^{\prime}}\right)$, for $n \gg 1$. The cycles $l^{\prime}$ satisfying this property are indexed by $\overline{\mathcal{S}_{i m}^{\prime}}$. 
(c) $l^{\prime} \in \mathcal{S}^{\prime} \backslash \overline{\mathcal{S}_{i m}^{\prime}}$.

Example 9.1.1 In general, $\mathcal{S}_{i m}^{\prime} \varsubsetneqq \overline{\mathcal{S}_{i m}^{\prime}}$. E.g. in Example 3.4.3, $\mathcal{O}_{Z}\left(-Z_{\text {min }}\right) \notin \operatorname{im}(c)$, however $\mathcal{O}_{Z}\left(-2 Z_{\text {min }}\right) \in \operatorname{im}(c)$. Furthermore, in general, $\overline{\mathcal{S}_{i m}^{\prime}} \varsubsetneqq \mathcal{S}^{\prime}$ either. Indeed, take e.g. a situation when $\operatorname{im}\left(c^{-l^{\prime}}\right)$ is a point different than $\mathcal{O}_{Z}\left(-l^{\prime}\right)$. Then $\mathcal{O}_{Z}\left(-n l^{\prime}\right) \notin$ $\operatorname{im}\left(c^{-n l^{\prime}}\right)$ for $n \geq 1$, hence $n l^{\prime} \notin \mathcal{S}_{i m}^{\prime}$ for $n \geq 1$. In such cases $\mathcal{S}^{\prime} \backslash \overline{\mathcal{S}_{i m}^{\prime}}$ is even infinite. For a concrete example see the last case of 3.4.1.

Lemma 9.1.2 Let $Z \geq E$ be an arbitrary cycle as above.

(a) Fix $l^{\prime} \in-\mathcal{S}^{\prime}$ as above, and assume that $n \geq 1$ satisfies the next assumptions:

(i) $\operatorname{im}\left(\widetilde{c}^{n l^{\prime}}\right)=A\left(n l^{\prime}\right)$ (automatically satisfied if $n$ is sufficiently large, cf. Theorem 6.1.9),

(ii) $0 \in \operatorname{im}\left(\widetilde{c}^{n l^{\prime}}\right)$.

Then $0 \in A\left(l^{\prime}\right)$ and $\operatorname{im}\left(\widetilde{c}^{m l^{\prime}}\right)=A\left(l^{\prime}\right)$ for any $m \geq n$.

(b) $\overline{\mathcal{S}_{i m}^{\prime}}=\mathcal{S}^{\prime}$ if and only if $\mathcal{S}^{\prime} \backslash \mathcal{S}_{i m}^{\prime}$ is finite.

Proof (a) Since $0 \in A\left(n l^{\prime}\right)$, by Theorem 6.1.9(a) necessarily $A\left(k l^{\prime}\right)=A\left(l^{\prime}\right)=V\left(l^{\prime}\right)$ for any $k \geq 1$. Fix $\mathcal{L} \in \operatorname{im}\left(\widetilde{c}^{k l^{\prime}}\right)$. Then, $\mathcal{L} \in A\left(k l^{\prime}\right)$ and by (6.1.2) and Lemma 6.1.6, $A\left(l^{\prime}\right)=A\left(l^{\prime}\right)+\mathcal{L} \subset \operatorname{im}\left(\widetilde{c}^{n l^{\prime}}\right)+\operatorname{im}\left(\widetilde{c}^{k l^{\prime}}\right) \subset \operatorname{im}\left(\widetilde{c}^{(n+k) l^{\prime}}\right) \subset A\left((n+k) l^{\prime}\right)=A\left(l^{\prime}\right)$. Part (b) follows from (a).

9.2. In the remaining part of this subsection we will work with line bundles defined over $Z \gg 0$.

Definition 9.2.1 (a) Following Neumann and Wahl [47], we say that $(X, o)$ and its resolution $\phi$ satisfy the End Curve Condition (ECC) if $E_{v}^{*} \in \mathcal{S}_{i m}^{\prime}$ for any end vertex $v \in \mathcal{V}$ (i.e. for $\delta_{v}=1$ ).

(b) We say that $(X, o)$ and its resolution $\phi$ satisfies the Weak End Curve Condition (WECC) if $E_{v}^{*} \in \overline{\mathcal{S}_{i m}^{\prime}}$ for any end vertex $v \in \mathcal{V}$.

If we restrict ourselves to singularities with rational homology sphere links, by End Curve Theorem [47] (see also [50]) singularities which satisfy ECC are exactly the splice quotient singularities of Neumann and Wahl [45]. The WECC terminology is new in the literature, however its necessity and importance appeared in many private discussions of the second author with Okuma in the last decade. The main question regarding singularities satisfying WECC is how can one generalize the results valid for splice quotient singularities to this larger family. The present article shows that e.g. the $p_{g}$-additivity formula of Okuma extends. Indeed, the general additivity formula (6.1.12) provides an additivity with correction term $\operatorname{dim} V_{\widetilde{X}}(I)$. Furthermore, as we will see in the next discussions, the correction term $\operatorname{dim} V_{\widetilde{X}}(I)$ has different reinterpretations in terms of certain Hilbert polynomials or Poincaré series (similarly as in the splice quotient case) whenever WECC is satisfied.

Proposition 9.2.2 (a) (Convexity property of $\overline{\mathcal{S}_{i m}^{\prime}}$ ) Fix $u, v \in \mathcal{V}, u \neq v$. If $E_{u}^{*}$, $E_{v}^{*} \in \overline{\mathcal{S}_{i m}^{\prime}}$ then for any vertex $w$ on the geodesic path in the graph connecting $u$ and $v$ one has $E_{w}^{*} \in \overline{\mathcal{S}_{i m}^{\prime}}$ too. 
(b) $(X, o)$ satisfies WECC if and only if $\overline{\mathcal{S}_{i m}^{\prime}}=\mathcal{S}^{\prime}$.

Proof Fix integers $n_{u}, n_{v}, n_{w}$ sufficiently large such that (i) $n_{u} E_{u}^{*}, n_{v} E_{v}^{*}, n_{w} E_{w}^{*}$ belong to $L$, (ii) the $E_{w}$-multiplicities of these three cycles are equal, and (iii) $n_{u} E_{u}^{*}$ and $n_{v} E_{v}^{*}$ belong to $\mathcal{S}_{i m}^{\prime}$. Set $l:=n_{u} E_{u}^{*}-n_{w} E_{w}^{*}$, and let the connected components of $\Gamma \backslash w$ be $\cup_{i} \Gamma_{i}$. We distinguish $\Gamma_{i_{0}}$, which contains $u$. Then $l$ is supported on $\cup_{i} \Gamma_{i}$. Since $\left(l, E_{z}\right)=0$ for any $z \in \mathcal{V}\left(\cup_{i \neq i_{0}} \Gamma_{i}\right), l \mid \Gamma_{i}=0$ for all $i \neq i_{0}$. Since $\left(l, E_{z}\right) \leq 0$ for any $z \in \mathcal{V}\left(\Gamma_{i_{0}}\right)$, and $\left(l, E_{u}\right)<0$, all the entries of $l \mid \Gamma_{i_{0}}$ are strict positive. We have similar property for $n_{v} E_{v}^{*}-n_{w} E_{w}^{*}$ too. Hence $\min \left\{n_{u} E_{u}^{*}, n_{v} E_{v}^{*}\right\}=n_{w} E_{w}^{*}$. Since, by assumption there exist functions $f_{u}$ and $f_{v}$, which can be regarded as sections of $\mathcal{O}\left(-n_{u} E_{u}^{*}\right)$ and $\mathcal{O}\left(-n_{v} E_{v}^{*}\right)$ without fixed components, the generic linear combination $a f_{u}+b f_{v}$ is a section of $\mathcal{O}\left(-n_{w} E_{w}^{*}\right)$ without fixed components. For $(b)$ use part $(a)$ and the fact that $\Gamma$ is a tree.

\subsection{Different reinterpretations of $\operatorname{dim}\left(V_{\widetilde{X}}\left(I^{\prime}\right)\right)$ when $I^{\prime} \in \overline{\mathcal{S}_{i m}^{\prime}}$}

In the sequel we apply the results of the previous section (e.g. Theorem 6.1.9) for natural line bundles. This will also include the 'classical' cases $\mathcal{L}=\mathcal{O}_{\widetilde{X}}(-l)$, where $l$ is an effective integral cycle. In order to do this we will need additional assumptions of type $\mathcal{L} \in \operatorname{im}\left(c^{n l^{\prime}}\right)$.

We fix the following setup. We consider line bundles over $\tilde{X}$, or over $Z \gg 0$. We write $V_{\widetilde{X}}\left(l^{\prime}\right)$ for the stabilized $V_{Z}\left(l^{\prime}\right)$ with $Z \gg 0$. We fix $l^{\prime} \in \mathcal{S}^{\prime}$ from $\overline{\mathcal{S}_{i m}^{\prime}}$, this means that there exists $n \gg 1$ such that $\mathcal{O}\left(-n l^{\prime}\right)$ admits sections without fixed components. Let $o \in \mathbb{Z}_{>0}$ be the order of $\left[l^{\prime}\right]$ in $L^{\prime} / L$. We also write $o l^{\prime}=l \in L$. Note that $V_{\widetilde{X}}\left(l^{\prime}\right)=V_{\widetilde{X}}\left(o l^{\prime}\right)$, cf. Lemma 6.1.6.

\section{$9.4 \operatorname{dim}\left(V_{\widetilde{X}}\left(I^{\prime}\right)\right)$ as the last coefficient of a Hilbert polynomial}

Consider the situation of Sect. 9.3. For $n \gg 1$ from the exact sequence of sheaves $0 \rightarrow \mathcal{O}_{\tilde{X}}(-n l) \rightarrow \mathcal{O}_{\tilde{X}} \rightarrow \mathcal{O}_{n l} \rightarrow 0$, we get

$$
\operatorname{dim} H^{0}(\mathcal{O}) / H^{0}(\mathcal{O}(-n l))=\chi(n l)-h^{1}(\mathcal{O}(-n l))+p_{g}(X, o),
$$

which combined with Theorem 6.1.9 gives

$$
\operatorname{dim} H^{0}(\mathcal{O}) / H^{0}(\mathcal{O}(-n l))=\chi(n l)+\operatorname{dim} V_{\widetilde{X}}(l)
$$

This already shows that $V_{\widetilde{X}}(l)$ is the free term of the Hilbert polynomial associated with $n \mapsto \operatorname{dim} H^{0}(\mathcal{O}) / H^{0}(\mathcal{O}(-n l))$. This fact can be reorganized even more. Note that by Theorem 6.1.9(d) $\mathcal{O}(-n l)$ is generated by global sections for all $n \geq n_{0}$ for some $n_{0}$. Therefore, if we denote the ideal $H^{0}\left(\widetilde{X}, \mathcal{O}\left(-n_{0} l\right)\right) \subset \mathcal{O}_{X, o}$ by $\mathcal{J}$, then the integral closure of its powers satisfy $\overline{\mathcal{J}^{m}}=H^{0}\left(\tilde{X}, \mathcal{O}\left(-m n_{0} l\right)\right)$ [27]. In particular, $\operatorname{dim}\left(\mathcal{O}_{X, o} / \overline{\mathcal{J}^{m}}\right)=\chi\left(m n_{0} l\right)+\operatorname{dim} V_{\widetilde{X}}(l)$.

Recall that there exist integral coefficients $\bar{e}_{i}(\mathcal{J})$ (where $i=1,2,3$ ) such that $\operatorname{dim}\left(\mathcal{O}_{X, o} / \overline{\mathcal{J}^{m}}\right)=\bar{e}_{0}(\mathcal{J})\left(\begin{array}{c}m+1 \\ 2\end{array}\right)-\bar{e}_{1}(\mathcal{J})\left(\begin{array}{c}m \\ 1\end{array}\right)+\bar{e}_{2}(\mathcal{J})$ for $m \gg 1$. Here, the polynomial 
from the right hand side is called the normal Hilbert polynomial of $\mathcal{J}$. One verifies that $\bar{e}_{2}(\mathcal{J})$ is independent of the choice of $n_{0}$. Then, the two identities combined provide $\operatorname{dim} V_{\widetilde{X}}(l)=\bar{e}_{2}(\mathcal{J})$.

If in our general identities from Theorem 6.1 .9 we insert $\bar{e}_{2}(\mathcal{J})$ for $\operatorname{dim} V_{\widetilde{X}}(l)$, then we recover e.g. the results from [52, §3]; or the additivity statement from [49, Cor. 4.5].

\section{$9.5 \operatorname{dim}\left(V_{\widetilde{X}}\left(I^{\prime}\right)\right)$ in terms of the multivariable series $P_{h=0}(t)$}

Assume again that $l^{\prime} \in \overline{\mathcal{S}_{i m}^{\prime}}$, and let $I$ be the $E_{v}^{*}$-support of $l^{\prime}$, that is, $l^{\prime}=\sum_{v \in I} a_{v} E_{v}^{*}$ with $a_{v} \in \mathbb{Z}_{>0}$. Then with the notations of 9.3 , for $n$ sufficiently large $\mathcal{O}\left(-n o l^{\prime}\right)$ has no fixed components and $h^{1}(\tilde{X}, \mathcal{O}(-n l))=p_{g}-\operatorname{dim} V_{\widetilde{X}}(I)$. This combined with (2.3.8) gives that for cycles of type $n l(n \gg 1)$

$$
\sum_{\tilde{l} \in L, \tilde{l} \nsupseteq n l} p_{\mathcal{O}(-\tilde{l})}=\chi(n l)+\operatorname{dim} V_{\widetilde{X}}(I) ;
$$

that is, the counting function $n l \mapsto \sum_{\tilde{l} \in L, \tilde{l} \nsupseteq n l} p_{\mathcal{O}(-\tilde{l})}$ of the coefficients of $P_{h=0}(\mathbf{t})$ is (for $n \gg 1$ ) the multivariable quadratic polynomial $\chi(n l)+\operatorname{dim} V_{\widetilde{X}}(I)$ in $n l$, whose free term is exactly $\operatorname{dim} V_{\widetilde{X}}(I)$.

The above counting function can be simplified even more: we will reduce the variables of $P_{0}$ to the variables indexed by $I$. For this we define the projection (along the $E$-coordinates) $\pi_{\mathcal{I}}: \mathbb{R}\left\langle E_{v}\right\rangle_{v \in \mathcal{V}} \rightarrow \mathbb{R}\left\langle E_{v}\right\rangle_{v \in \mathcal{I}}$, denoted also as $\left.x \mapsto x\right|_{\mathcal{I}}$, by $\sum_{v \in \mathcal{V}} l_{v} E_{v} \mapsto \sum_{v \in \mathcal{I}} l_{v} E_{v}$.

For further motivations and topological analogues of the next statements see also [23] (where $Z(\mathbf{t})$ plays the role of $P(\mathbf{t})$ ).

Lemma 9.5.2 Assume that $l^{\prime}=\sum_{v \in I} a_{v} E_{v}^{*}$ with $a_{v}>0$, and $l^{\prime \prime} \in \mathcal{S}^{\prime}$ too. Then $l^{\prime \prime} \geq l^{\prime}$ if and only if $\left.l^{\prime \prime}\right|_{I} \geq\left. l^{\prime}\right|_{I}$.

Proof We prove the $\Leftarrow$ part. Write $l^{\prime \prime}-l^{\prime}$ as $x+y$, where $x$ (resp $y$ ) is supported on $E_{I}$ (resp. on $\left.E_{\mathcal{V} \backslash I}\right)$. By assumption, $x \geq 0$. For any $u \in \mathcal{V} \backslash I$ one has $0 \geq\left(l^{\prime \prime}, E_{u}\right)=$ $\left(l^{\prime}, E_{u}\right)+\left(x, E_{u}\right)+\left(y, E_{u}\right)$. But $\left(l^{\prime}, E_{u}\right)=0$ and $\left(x, E_{u}\right) \geq 0$. Hence $\left(y, E_{u}\right) \leq 0$ for any $u$ in the support of $y$. Since (, ) is negative definite, $y \geq 0$.

According to the $\pi_{I}$ projection, we also define the series $P_{I, h}\left(\mathbf{t}_{I}\right)$ (for any $h \in H$ ), in variables $\left\{t_{v}\right\}_{v \in I}$ by $P_{I, h}\left(\mathbf{t}_{I}\right):=\left.P_{h}(\mathbf{t})\right|_{t_{v}=1, v \notin I}$.

Note that the series $P_{I, 0}\left(\mathbf{t}_{I}\right)$ has the form $\sum_{l_{I} \in \pi_{I}\left(\mathcal{S}^{\prime} \cap L\right)} p_{I}\left(l_{I}\right) \mathbf{t}_{I}^{l_{I}}$. By Lemma 9.5.2 one has

$$
\sum_{\tilde{l} \in L, \tilde{l} \ngtr n l} p_{\mathcal{O}(-\tilde{l})}=\sum_{l_{I} \in \pi_{I}(L),\left.l_{I} \ngtr n l\right|_{I}} p_{I}\left(l_{I}\right) .
$$


Therefore, for $n \gg 1$, one also has that the counting function of the coefficients of the reduced series $P_{I, 0}$ provides the same expression

$$
\sum_{l_{I} \in \pi_{I}(L),\left.l_{I} \ngtr n l\right|_{I}} p_{I}\left(l_{I}\right)=\chi(n l)+\operatorname{dim} V_{\widetilde{X}}(I) .
$$

(Note that if the $E^{*}$-support of $n l$ is $I$, then $\left.n l\right|_{I}$ determines uniquely $n l$.)

E.g., if $I=\{v\}$ (under the assumption $E_{v}^{*} \in \overline{\mathcal{S}_{i m}^{\prime}}$ ), $P_{I, 0}=\sum_{m \geq 0} p_{v}(m) t_{v}^{m}$ has only one variable, and $\sum_{m \geq\left. n l\right|_{v}} p_{v}(m)=\chi(n l)+\operatorname{dim} V_{\widetilde{X}}(I)$ for $n \gg 1$.

Theorem 9.5.4 Assume that $(X, o)$ is a splice quotient singularity associated with the graph $\Gamma$ (or, equivalently, $\phi: \widetilde{X} \rightarrow X$ satisfies the ECC, cf. Definition 9.2.1). Then for any $I$ the dimension $\operatorname{dim} V_{\widetilde{X}}(I)$ is topological, computable from $\Gamma$.

Proof For splice quotient singularities $P(\mathbf{t})$ equals the topological series $Z(\mathbf{t})$, cf. [37]. Hence, in (9.5.1) the left hand side can be replaced by the corresponding sum of the coefficients of $Z(\mathbf{t})$.

Remark 9.5.5 Let us denote the Seiberg-Witten invariant of the link $M(\Gamma)$, associate with the canonical $\operatorname{spin}^{c}$-structure of $M(\Gamma)$ with $\mathfrak{s w}_{\text {can }}(M(\Gamma))$, and the corresponding normalized Seiberg-Witten invariant by $\overline{\mathfrak{s w}}_{\text {can }}(M(\Gamma)):=\mathfrak{s w}_{\text {can }}(M(\Gamma))+\left(Z_{K}^{2}+\right.$ $|\mathcal{V}(\Gamma)|) / 8$, see e.g. [23]. Recall also that in the splice quotient case $P(\mathbf{t})=Z(\mathbf{t})$ (cf. [37]). Therefore, if we replace in (9.5.3) $P(\mathbf{t})$ by $Z(\mathbf{t})$, in the terminology of [23] (9.5.3) reads as follows: $\operatorname{dim} V_{\widetilde{X}}(I)$ is the periodic constant of the $I$-reduction $Z_{I, 0}\left(\mathbf{t}_{I}\right)$ of $Z_{0}(\mathbf{t})$, and by Theorem 3.1.1 of [23] it equals $-\overline{\mathfrak{s w}}_{c a n}(M(\Gamma))+\overline{\mathfrak{s w}}_{c a n}(M(\Gamma \backslash I))$.

\subsection{The equivariant version of 9.5}

Note that the identity $(\dagger) h^{1}\left(\tilde{X}, \mathcal{O}\left(-n l^{\prime}\right)\right)=p_{g}-\operatorname{dim} V_{\widetilde{X}}(I)$ holds uniformly for any $n \gg 1$, though $\left[n l^{\prime}\right] \in H$ might have different $H$-classes. Such stability usually cannot be proved via cohomology exact sequence of type $\left.0 \rightarrow \mathcal{L}(-l) \rightarrow \mathcal{L} \rightarrow \mathcal{L}\right|_{l} \rightarrow 0, l \in$ $L_{>0}$ (since in this situation $c_{1}(\mathcal{L}(-l))-c_{1}(\mathcal{L}) \in L$ ), or by eigenspace decomposition of some sheaf associated with the universal abelian cover $\left(X_{a b}, o\right)$. Maybe one should emphasize that in the above identity $(\dagger)$ the contribution $p_{g}$ comes from the dimension of Pic ${ }^{l^{\prime}}$, which is independent of the class $\left[l^{\prime}\right] \in H$, and not from the $p_{g}\left(X_{a b}, o\right)_{h}$ for $h=0$.

Now, if we apply (2.3.8) for $(\dagger)$ for different classes we obtain the following fact. Let us fix, as above $l^{\prime} \in \overline{\mathcal{S}_{i m}^{\prime}}$ with $E^{*}$-support $I$, and let us fix also some $k \in \mathbb{Z}_{\geq 0}$, $h:=\left[k l^{\prime}\right] \in H$, and write $k l^{\prime}=r_{h}+l_{k}$ for some $l_{k} \in L$. Let $o$ be the order of $\left[l^{\prime}\right]$ in $H$ as above. Then from (2.3.8) one has

$$
\begin{aligned}
h^{1}\left(\mathcal{O}\left(-r_{h}-l_{k}-n o l^{\prime}\right)=\right. & -\sum_{a \in L, a \nsupseteq 0} p_{\mathcal{O}\left(-r_{h}-l_{k}-n o l^{\prime}\right)} \\
& +p_{g}\left(X_{a b}, o\right)_{h}+\chi\left(l_{k}+n o l^{\prime}\right)-\left(l_{k}+n o l^{\prime}, r_{h}\right) .
\end{aligned}
$$


or, for any $k$ and any $n \gg 1$,

$$
\begin{aligned}
\sum_{a \in L, a \nsupseteq 0} p_{\mathcal{O}\left(-r_{h}-l_{k}-n o l^{\prime}\right)}= & \chi\left(l_{k}+n o l^{\prime}\right)-\left(l_{k}+n o l^{\prime}, r_{h}\right) \\
& +p_{g}\left(X_{a b}, o\right)_{h}-p_{g}+\operatorname{dim} V_{\widetilde{X}}(I) .
\end{aligned}
$$

Hence $\operatorname{dim} V_{\widetilde{X}}(I)$ connects the asymptotic behaviour of different $h$-components of $P(\mathbf{t})$ of the form $h=\left[k l^{\prime}\right], k \in \mathbb{Z}$.

\section{The 'non-stable' $\operatorname{dim} \operatorname{im}\left(c^{\prime \prime}\right)$ and differential forms}

10.1. The first theorem of this section is a generalization of that statement of Sect. 8, which says that for $Z \geq E$ the dual of the vector subspace $V_{Z}\left(n l^{\prime}\right) \subset H^{1}\left(\mathcal{O}_{Z}\right)$, the 'stable image affine subspace' $\operatorname{im}\left(\widetilde{c}^{n l^{\prime}}\right)=A_{Z}\left(n l^{\prime}\right)(n \gg 1)$ shifted to the origin, agrees with the subspace of forms $\Omega_{Z}(I)$, where $I$ is the $E^{*}$-support of $l^{\prime}$ (see Theorem 8.1.3 and Sect. 8.3). $V_{Z}\left(n l^{\prime}\right)$ can also be interpreted (up to a shift) as the tangent space at any $\mathcal{L} \in A_{Z}\left(n l^{\prime}\right)$ of $A_{Z}\left(n l^{\prime}\right)$. Hence, $\mathcal{L}+V_{Z}\left(n l^{\prime}\right)$ is the intersection of all the kernels of linear maps $T_{\mathcal{L}} \omega$, where $\omega \in \Omega_{Z}(I)$ (that is, for all $\omega$ without pole along those $E_{v}$ 's which support the divisors from $\mathrm{ECa}^{n l^{\prime}}(Z)$ ). For the explicit description of the duality see 7.1 .

The new setup is the following. Consider a divisor $D \in \mathrm{ECa}^{l^{\prime}}(Z)$, which is a union of $\left(l^{\prime}, E\right)$ disjoint divisors $\left\{D_{i}\right\}_{i}$, each of them $\mathcal{O}_{Z}$-reduction of divisors $\left\{\widetilde{D}_{i}\right\}_{i}$ from $\operatorname{ECa}^{l^{\prime}}(\widetilde{X})$ intersecting $E$ transversally. Set $\widetilde{D}=\cup_{i} \widetilde{D}_{i}$ and $\mathcal{L}:=\widetilde{c}^{l^{\prime}}(D) \in H^{1}\left(Z, \mathcal{O}_{Z}\right)$. Set also $Z=\sum_{v} m_{v} E_{v}$.

We introduce a subsheaf $\Omega_{\widetilde{X}}^{2}(Z)^{\operatorname{regRes}} \widetilde{D}$ of $\Omega_{\widetilde{X}}^{2}(Z)$ consisting of those forms $\omega$ which have the property that the residue $\operatorname{Res} \widetilde{D}_{i}(\omega)$ has no poles along $\widetilde{D}_{i}$ for all $i$. This means that the restrictions of $\Omega_{\widetilde{X}}^{2}(Z)^{\text {regRes } \widetilde{D}}$ and $\Omega_{\widetilde{X}}^{2}(Z)$ on the complement of the support of $\widetilde{D}$ coincide, however along $\widetilde{D}$ is satisfies the following requirement. If $p=E \cap \widetilde{D}_{i}=$ $E_{v_{i}} \cap \widetilde{D}_{i}$ has local coordinates $(u, v)$ with $\{u=0\}=E$ and $\widetilde{D}_{i}$ with local equation $v$, then a local section of $\Omega_{\widetilde{X}}^{2}(Z)$ near $p$ has the form $\omega=\sum_{i \geq-m_{v_{i}}, j \geq 0} a_{i, j} u^{i} v^{j} d u \wedge d v$. Then the residue $\operatorname{Res}_{\widetilde{D}_{i}}(\omega)$ is $\left.(\omega / d v)\right|_{v=0}=\sum_{i} a_{i, 0} u^{i} d u$, hence the pole-vanishing reads as $a_{i, 0}=0$ for all $i<0$. Note that $\Omega_{\widetilde{X}}^{2}(Z-\widetilde{D})$ and the sheaf of regular forms $\Omega_{\widetilde{X}}^{2}$ are subsheaves of $\Omega_{\widetilde{X}}^{2}(Z)^{\text {regRes } \widetilde{D}}$.

Theorem 10.1.1 In the above situation one has the following facts.

(a) The sheaves $\Omega_{\widetilde{X}}^{2}(Z)^{\operatorname{regRes} \widetilde{D}} / \Omega_{\widetilde{X}}^{2}$ and $\mathcal{O}_{Z}\left(K_{\widetilde{X}}+Z-D\right)$ are isomorphic.

(b) $H^{0}\left(\widetilde{X}, \Omega_{\widetilde{X}}^{2}(Z)^{\operatorname{regRes}} \widetilde{D}\right) / H^{0}\left(\widetilde{X}, \Omega_{\widetilde{X}}^{2}\right) \simeq H^{1}(Z, \mathcal{L})^{*}$. (The left hand side can be regarded as a subspace of $\left.H^{0}\left(\tilde{X}, \Omega_{\widetilde{X}}^{2}(Z)\right) / H^{0}\left(\tilde{X}, \Omega_{\widetilde{X}}^{2}\right) \simeq H^{1}\left(Z, \mathcal{O}_{Z}\right)^{*}\right)$.

(c) The image $T_{D} \widetilde{c}^{l^{\prime}}\left(T_{D} \underset{\widetilde{E C a}}{l^{\prime}}(Z)\right)$ of the tangent map $T_{D} \widetilde{c}^{l^{\prime}}$ at $D$ of $\widetilde{c}^{l^{\prime}}: \mathrm{ECa}^{l^{\prime}}(Z) \rightarrow$ $H^{1}\left(Z, \mathcal{O}_{Z}\right)=H^{1}\left(\widetilde{X}, \mathcal{O}_{\widetilde{X}}\right)$ is the intersection of kernels of linear maps $T_{\mathcal{L}} \omega:$ $T_{\mathcal{L}} H^{1}\left(\widetilde{X}, \mathcal{O}_{\tilde{X}}\right) \rightarrow \mathbb{C}$, where $\omega \in H^{0}\left(\widetilde{X}, \Omega_{\widetilde{X}}^{2}(Z)^{\operatorname{regRes} \widetilde{D}}\right)$. 
Proof (a) Consider the following diagram:

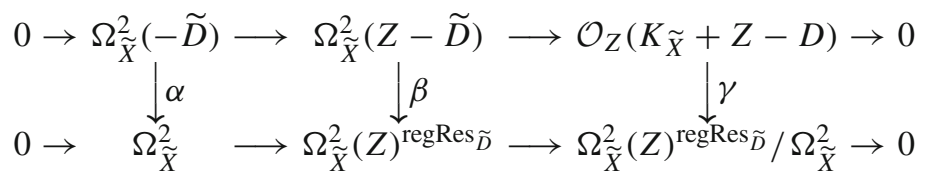

Above $\alpha$ and $\beta$ are the natural inclusions. We claim that their cokernels are isomorphic. Indeed, with the notation $M_{i, j}=u^{i} v^{j} d u \wedge d v$ one has $\operatorname{coker}(\alpha)=$ $\left\{\sum_{j \geq 0, i \geq 0} a_{i, j} M_{i, j}\right\} /\left\{\sum_{j \geq 1, i \geq 0} a_{i, j} M_{i, j}\right\}$ and $\operatorname{coker}(\beta)=\left\{\sum_{j \geq 0, i \geq-m_{v_{i}}} a_{i, j}\right.$ $\left.M_{i, j} \mid a_{i<0,0}=0\right\} /\left\{\sum_{j \geq 1, i \in \mathbb{Z}} a_{i, j} M_{i, j}\right\}$. Hence $\gamma$ is an isomorphism.

(b) Since $H^{1}\left(\widetilde{X}, \Omega_{\widetilde{X}}^{2}\right)=0$, by part $(a)$ we have $H^{0}\left(\widetilde{X}, \Omega_{\widetilde{X}}^{2}(Z)^{\operatorname{regRes}} \widetilde{D}\right) / H^{0}\left(\widetilde{X}, \Omega_{\widetilde{X}}^{2}\right)$ $=H^{0}\left(\mathcal{O}_{Z}\left(K_{\tilde{X}}+Z-D\right)\right)$. But, this last one equals $H^{1}\left(Z, \mathcal{O}_{Z}(D)\right)^{*}$ by Serre duality.

(c) We prove the statement in the case $\left(l^{\prime}, E\right)=1$, the general case follows similarly. Hence, set $l^{\prime}=-E_{v}^{*}$ for some vertex $v \in \mathcal{V}$, that is, $\widetilde{D}$ is a transversal cut at the point $p$ of the exceptional divisor $E_{v}$. Consider local coordinates $(u, v)$ around $p$ as above. Recall that the local equation of $D$ is $v$. Let $\left\{\widetilde{D}_{t}\right\}_{t \in \mathbb{C},|t| \ll 1}$ be a path in $\mathrm{ECa}^{l^{\prime}}$ at $D$ whose local equation is $v+t u^{o-1}$ for some $o \geq 1$.

Consider also an arbitrary form $\omega \in H^{0}\left(\widetilde{X}, \Omega_{\widetilde{X}}^{2}(Z)\right)$ (with local equation as above). Then (the class of) $\omega$ is in the dual space of the image $T_{D} \widetilde{c}^{l^{\prime}}\left(T_{D} \mathrm{ECa}{ }^{l^{\prime}}(Z)\right)$ if and only if $\left(T_{\mathcal{L}} \omega\right)\left(T_{D} \widetilde{c}^{\prime}(\delta)\right)=0$ for all tangent vectors $\delta$, the tangent vectors of paths of type $D_{t}$ at $D$. But $T_{\mathcal{L}} \omega\left(T_{D} \widetilde{c}^{\prime}(\delta)\right)=\lambda \cdot a_{-o, 0}(\lambda \neq 0)$ by 7.2.3. Therefore, the dual space of forms is exactly the class of forms from $H^{0}\left(\widetilde{X}, \Omega_{\widetilde{X}}^{2}(Z)^{\operatorname{regRes}} \widetilde{D}\right)$.

In fact, one also sees that the dimensions of these two spaces $\operatorname{im}\left(T_{D} \widetilde{c}\right)$ and $\cap_{\omega} T_{\mathcal{L}} \omega$ agree. Indeed, $\operatorname{dim} \operatorname{im}\left(T_{D} \widetilde{c}\right)=h^{1}\left(\mathcal{O}_{Z}\right)-h^{1}(Z, \mathcal{L})$ by (3.1.8). But, $\operatorname{dim} \cap_{\omega} T_{\mathcal{L}} \omega$ is the same by $(b)$.

Corollary 10.1.2 Assume that $\left\{\omega_{1}, \ldots, \omega_{h}\right\}$ form a basis of $H^{0}\left(\widetilde{X}, \Omega_{\widetilde{X}}^{2}(Z)\right) /$ $H^{0}\left(\tilde{X}, \Omega_{\widetilde{X}}^{2}\right)$. Then

$$
\begin{aligned}
& H^{0}\left(\tilde{X}, \Omega_{\widetilde{X}}^{2}(Z)^{\operatorname{regRes} \widetilde{D}}\right) / H^{0}\left(\tilde{X}, \Omega_{\widetilde{X}}^{2}\right) \\
& \quad=\left\{\left(a_{1}, \ldots, a_{h}\right) \in \mathbb{C}^{h}: \operatorname{Res}_{\widetilde{D}_{i}}\left(\sum_{\alpha} a_{\alpha} \omega_{\alpha}\right) \text { has no pole along } \widetilde{D}_{i} \text { for all } i\right\} .
\end{aligned}
$$

Hence, by Theorem 10.1.1, the dimension of the right hand side is $h^{1}(Z, \mathcal{L})$, and the number of independent relations between $\left(a_{1}, \ldots, a_{h}\right), h^{1}\left(\mathcal{O}_{Z}\right)-h^{1}(Z, \mathcal{L})$, is the dimension of $\operatorname{im} T_{D} c^{l^{\prime}}\left(T_{D} \mathrm{ECa}^{l^{\prime}}(Z)\right)$.

In particular, $\operatorname{dim}\left(\operatorname{im}\left(c^{l^{\prime}}(Z)\right)\right)$ is the number of independent relations for $\left\{\widetilde{D}_{i}\right\}_{i}$ generic.

10.2. The above theorem can be applied rather directly in several situations, when we can provide a bases for $H^{1}\left(Z, \mathcal{O}_{Z}\right)^{*}=H^{0}\left(\widetilde{X}, \Omega_{\widetilde{X}}^{2}(Z)\right) / H^{0}\left(\widetilde{X}, \Omega_{\widetilde{X}}^{2}\right)$, and verify directly for certain (or for all) divisors $D$ the above pole-vanishing property. In the next subsections we provide such applications. 


\subsection{The Gorenstein case}

Assume that $(X, o)$ is Gorenstein, fix a resolution $\tilde{X} \rightarrow X$ as above, and let $\omega_{0} \in H^{0}\left(\widetilde{X}, \Omega_{\widetilde{X}}^{2}\left(Z_{K}\right)\right)$ be the pullback of the Gorenstein form, well defined up to a non-zero constant. Its pole is $Z_{K}$, the (anti)canonical cycle. Since $\Omega_{\widetilde{X}}^{2}=\mathcal{O}_{\widetilde{X}}\left(-Z_{K}\right.$ ), $H^{0}\left(\tilde{X}, \Omega_{\widetilde{X}}^{2}\left(Z_{K}\right)\right) / H^{0}\left(\widetilde{X}, \Omega_{\widetilde{X}}^{2}\right)$ is isomorphic with $H^{0}\left(\widetilde{X}, \mathcal{O}_{\tilde{X}}\right) / H^{0}\left(\widetilde{X}, \mathcal{O}_{\widetilde{X}}\left(-Z_{K}\right)\right)$, hence if we fix a basis of $H^{0}\left(\tilde{X}, \mathcal{O}_{\tilde{X}}\right) / H^{0}\left(\tilde{X}, \mathcal{O}_{\widetilde{X}}\left(-Z_{K}\right)\right)$ consisting of classes of functions $\left\{f_{1}, \ldots, f_{p_{g}}\right\} \subset H^{0}\left(\widetilde{X}, \mathcal{O}_{\tilde{X}}\right)$ with divisors $\operatorname{div}_{E} f_{\alpha} \nsucceq Z_{K}$ then in $H^{0}\left(\tilde{X}, \Omega_{\widetilde{X}}^{2}(Z)\right) / H^{0}\left(\widetilde{X}, \Omega_{\widetilde{X}}^{2}\right)$ the classes of forms $\left\{f_{1} \omega_{0}, \ldots, f_{p_{g}} \omega_{0}\right\}$ form a basis.

Therefore, for any fixed $I \subset \mathcal{V}$,

$$
\Omega(I)=\left\{\left(a_{1}, \ldots, a_{p_{g}}\right) \in \mathbb{C}^{p_{g}}: m_{E_{v}}\left(\sum_{\alpha} a_{\alpha} f_{\alpha}\right) \geq m_{E_{v}}\left(Z_{K}\right) \text { for any } v \in I\right\},
$$

where $m_{E_{v}}(\cdot)$ denotes the coefficient of a cycle along $E_{v}$.

By Theorem 6.1.9 $\operatorname{dim} \Omega(I)=h^{1}(\widetilde{X}, \mathcal{L})$ for any $\mathcal{L}$ with $c_{1}(\mathcal{L})=n l^{\prime}$ with $n \gg 1$ and where $I:=\left\{E^{*}\right.$-support of $\left.l^{\prime}\right\}$. Furthermore, the number of independent relations between $\left(a_{1}, \ldots, a_{p_{g}}\right), p_{g}-\operatorname{dim} \Omega(I)$, is the dimension of the stable $\operatorname{im}\left(c^{n l^{\prime}}\right)(n \gg 1)$.

According to Theorem 10.1.1, these facts have the following generalizations. Set $\widetilde{D}=\cup_{i} \widetilde{D}_{i}$ be a divisor as in 10.1: each $\widetilde{D}_{i}$ is a transversal cut intersecting $E_{v(i)}$. Let $\gamma_{i}$ : $(\mathbb{C}, 0) \rightarrow\left(\widetilde{D}_{i}, \widetilde{D}_{i} \cap E_{v(i)}\right), t \mapsto \gamma_{i}(t)$, be a parametrization (local diffeomorphism). Set $\mathcal{L}=\mathcal{O}_{\widetilde{X}}(D)$ and $c_{1}(\mathcal{L})=l^{\prime}$.

Theorem 10.3.2 With the above notations one has

$$
\begin{aligned}
& H^{0}\left(\tilde{X}, \Omega_{\widetilde{X}}^{2}(Z)^{\operatorname{regRes} \widetilde{D}}\right) / H^{0}\left(\widetilde{X}, \Omega_{\widetilde{X}}^{2}\right) \\
& \quad=\left\{\left(a_{1}, \ldots, a_{p_{g}}\right) \in \mathbb{C}^{p_{g}}: \operatorname{ord}_{t}\left(\sum_{\alpha} a_{\alpha} f_{\alpha} \circ \gamma_{i}\right) \geq m_{E_{v(i)}}\left(Z_{K}\right) \text { for all } i\right\} .
\end{aligned}
$$

Similarly as in Corollary 10.1.2, the dimension of the right hand side is $h^{1}(\tilde{X}, \mathcal{L})$, and the number of independent relations between $\left(a_{1}, \ldots, a_{p_{g}}\right), p_{g}-h^{1}(\widetilde{X}, \mathcal{L})$, is the dimension of $\operatorname{im} T_{D} c^{l^{\prime}}\left(T_{D} \mathrm{ECa}^{l^{\prime}}(Z)\right)(Z \gg 0)$, and $\operatorname{dim}\left(\operatorname{im}\left(c^{l^{\prime}}\right)\right)$ is the number of independent relations for $\left\{\widetilde{D}_{i}\right\}_{i}$ generic.

We will apply this theorem in Sect. 11 for superisolated (hypersurface, hence Gorenstein) germs. The general non-Gorenstein case (that is, Corollary 10.1.2) will be exemplified in Sect. 12 on the case of weighted homogeneous germs, in which case we construct a concrete basis $\left\{\omega_{1}, \ldots, \omega_{p_{g}}\right\}$.

\section{Superisolated singularities}

\subsection{The setup}

We will exemplify the Gorenstein case on a special family of isolated hypersurface singularities. The family of superisolated singularities creates a bridge between the 
theory of projective plane curves and the theory of surface singularities. This bridge will be present in the next discussions as well. For details and results regarding such germs see e.g. [28,29].

Assume that $(X, o)$ is a hypersurface superisolated singularity. This means that $(X, o)$ is a hypersurface singularity $\left\{F\left(x_{1}, x_{2}, x_{3}\right)=0\right\}$, where the homogeneous terms $F_{d}+F_{d+1}+\cdots$ of $F$ satisfy the following properties: $\left\{F_{d}=0\right\}$ is reduced and it defines in $\mathbb{C P}^{2}$ an irreducible rational cuspidal curve $C$; furthermore, the intersection $\left\{F_{d+1}=0\right\} \cap \operatorname{Sing}\left\{F_{d}=0\right\}$ in $\mathbb{C P}^{2}$ is empty. The restrictions regarding $F_{d}$ implies that the link of $(X, o)$ is a rational homology sphere (this fact motivates partly the presence of these restrictions). With $F_{d}$ fixed, all the possible choices for $\left\{F_{i}\right\}_{i>d}$ define an equisingular family of singularities with fixed topology and fixed $p_{g}=$ $d(d-1)(d-2) / 6$. For simplicity, here we will take for $F_{d+1}$ the $(d+1)^{t h}$-power of some linear function and $F_{i}=0$ for $i>d+1$. Moreover, by linear change of variables, we can assume $F_{d+1}=-x_{3}^{d+1}$. (Note that in our treatment the analytic type of the singularity plays a crucial role, hence, by the choice $F_{d+1}=-x_{3}^{d+1}$ we restrict ourselves to a special analytic family. We do this since in this case the presentation of the next subsections are more transparent. However, it would be interesting to analyse the stability/non-stability of the Abel map in the whole equisingular family when we vary $F_{i}, i \geq d+1$.)

If we blow up the origin of $\mathbb{C}^{3}$ then the strict transform $X^{\prime}$ of $X$ is already smooth (this property is responsible for the name 'superisolated') —-hence a minimal resolution of $X$-the exceptional curve $C^{\prime} \subset X^{\prime}$ is irreducible and it can be identified with $C$ [28]. Hence, resolving the plane curve singularities of $C^{\prime}$ we get a minimal good resolution of $X$; for the precise resolution graph see e.g. [28,29]. In the minimal (or the minimal good) resolution the exceptional curve corresponding to $C^{\prime}$ will be denoted by $E_{0}$.

In the chart $x_{1}=u w, x_{2}=v w, x_{3}=w$ of the blow up the total transform has equation $w^{d}\left(w-F_{d}(u, v, 1)\right)=0, X^{\prime}=\left\{w=F_{d}(u, v, 1)\right\}, C^{\prime}=\{w=$ $\left.F_{d}(u, v, 1)=0\right\}$.

We wish to discuss the Abel map associated with several choices of $l^{\prime}$ and $Z$.

\subsection{The case $l^{\prime}=-k E_{0}^{*}(k \geq 1), Z=Z_{K}$ (and generic divisor on $\left.\mathrm{ECa}^{I^{\prime}}(Z)\right)$}

In this case a generic point $D$ of $\mathrm{ECa}^{l^{\prime}}(Z)$ consists of $k$ transversal cuts of $E_{0}$ at generic points. In order to determine $\operatorname{dimim}\left(c^{l^{\prime}}\right)$, which equals $\operatorname{dim} \operatorname{im} T_{D} \widetilde{c}^{\prime}$ $\left(T_{D} \mathrm{ECa}^{l^{\prime}}(Z)\right)$, we will apply Theorem 10.3.2. Hence, we need to analyse the restriction of forms on the components of the divisor $D$. Note that Theorem 10.3.2 automatically provides $h^{1}\left(Z_{K}, \mathcal{O}(D)\right)$ too. Furthermore, by Grauert-Riemenschneider vanishing $h^{1}\left(\widetilde{X}, \mathcal{O}\left(\widetilde{D}-Z_{K}\right)\right)=0$, one also has $h^{1}\left(Z_{K}, \mathcal{O}(D)\right)=h^{1}(\widetilde{X}, \mathcal{O}(\widetilde{D}))$.

Since the first blow up already creates the exceptional divisor $C^{\prime}=E_{0}$, all the computation can be done in this minimal resolution $\phi: X^{\prime} \rightarrow X$, and we can even assume that $D$ is in the chart considered above. First, we find $\left\{f_{\alpha}\right\}_{\alpha=1}^{p_{g}}$ such that $\left\{f_{1} \omega_{0}, \ldots, f_{p_{g}} \omega_{0}\right\}$ induces a basis in $H^{0}\left(\widetilde{X}, \Omega_{\widetilde{X}}^{2}(Z)\right) / H^{0}\left(\widetilde{X}, \Omega_{\widetilde{X}}^{2}\right)$. Notice that the pullback of any monomial $\mathbf{x}^{\mathbf{m}}=x_{1}^{m_{1}} x_{2}^{m_{2}} x_{3}^{m_{3}}$ has vanishing order $\operatorname{deg}\left(\mathbf{x}^{\mathbf{m}}\right)=\sum_{i} m_{i}=$ $|\mathbf{m}|$ along $E_{0}$. Moreover, the multiplicity of $Z_{K}$ along $C^{\prime}$ is $d-2$. Since the number 
of monomials of degree strict less than $d-2$ is $p_{g}=d(d-1)(d-2) / 6$, the set $\left\{\mathbf{x}^{\mathbf{m}}: \operatorname{deg}\left(\mathbf{x}^{\mathbf{m}}\right) \leq d-3\right\}$ serve as a basis for $H^{0}\left(\widetilde{X}, \mathcal{O}_{\tilde{X}}\right) / H^{0}\left(\widetilde{X}, \mathcal{O}_{\tilde{X}}\left(-Z_{K}\right)\right)$.

Next, we consider parametrizations of each component $\left\{\widetilde{D}_{i}\right\}_{i=1}^{k}$ (the liftings of the divisors $\left.\left\{D_{i}\right\}_{i}\right), t \mapsto \gamma_{i}(t)=\left(u_{i}(t), v_{i}(t), w_{i}(t)\right) \subset X^{\prime}$. In fact, we can start with a parametrization $t \mapsto\left(u_{i}(t), v_{i}(t)\right)$ of a transversal cut of $\left\{F_{d}(u, v, 1)=0\right\} \subset \mathbb{C}^{2}$ at some smooth point. Then we lift it to $X^{\prime}$ by setting $w_{i}(t):=f\left(u_{i}(t), v_{i}(t), 1\right)$. The tranversality implies that $w_{i}(t)$ has the form $c_{1} t+c_{2} t^{2}+\cdots$ with $c_{1} \neq 0$, hence after a reparametrization with $t^{\prime}:=w_{i}(t)$, we can assume that $w_{i}(t)=t$.

We denote the point $\left(u_{i}(0), v_{i}(0)\right) \in\left\{F_{d}(u, v, 1)=0\right\} \subset \mathbb{C}^{2}$ by $p_{i}$. We abridge $(u, v)^{\mathbf{m}}\left(p_{i}\right):=u_{i}(0)^{m_{1}} v_{i}(0)^{m_{2}}$. Then, the restriction of a monomial $\mathbf{x}^{\mathbf{m}}$ to $\widetilde{D}_{i}$ is

$$
u(t)^{m_{1}} v(t)^{m_{2}} t^{|\mathbf{m}|}=t^{|\mathbf{m}|}\left((u, v)^{\mathbf{m}}\left(p_{i}\right)+H_{\mathbf{m}}(t)\right),
$$

where $H_{\mathbf{m}}(t)$ denotes the 'higher order terms' with $H_{\mathbf{m}}(0)=0$. Hence, by Theorem 10.3.2,

$$
\begin{aligned}
h^{1}\left(Z_{K}, \mathcal{O}(D)\right)= & \operatorname{dim}\left\{\left(a_{\mathbf{m}}\right)_{\mathbf{m}} \in \mathbb{C}^{p_{g}}: \sum_{\mathbf{m}} a_{\mathbf{m}}\right. \\
& \cdot \frac{(u, v)^{\mathbf{m}}\left(p_{i}\right)+H_{\mathbf{m}}}{\left.t^{d-2-|\mathbf{m}|} \text { has no pole for all } i\right\} .}
\end{aligned}
$$

Expanding the sum into its Laurent series in $t$, and separating the coefficients of $\left\{t^{-d+2+j}\right\}_{0 \leq j \leq d-3}$, we get for each $D_{i}$ a linear system with $d-2$ equations for the variable $\left(a_{\mathbf{m}}\right)_{\mathbf{m}}$. We need to determine the rank of the corresponding matrix. This matrix has a natural block decomposition, a block is indexed by $j$ and the set $\mathbf{m}$ with fixed $|\mathbf{m}|$. We prefer to order the rows by $t^{-d+2}, t^{-d+3}, \ldots, t^{-1}$.

E.g., for fixed $D_{i}$, the first row has its first entry 1 (corresponding to the block $t^{-d+2}$ and $|\mathbf{m}|=0$ ) and all other entries zero. The second row has some entry in the first place, the second block corresponding to $t^{-d+3}$ and $\left.|\mathbf{m}|=1\right)$ has three entries, namely $u\left(p_{i}\right), v\left(p_{i}\right), 1$ (which are the evaluations of the degree $\leq 1(u, v)$-monomials at $p_{i}$ ), and the blocks corresponding to $|\mathbf{m}|>1$ are zero. More generally, above the diagonal all the blocks are zero, the diagonal block indexed by $t^{-d+2+j}$ and $|\mathbf{m}|=j$ contains the evaluation of the $(u, v)$-monomials of degree $\leq j$ at $p_{i}$.

E.g., if $k=1$, then the matrix has $d-2$ rows and $p_{g}$ columns, and each diagonal block contains one entry 1 , hence its rank of the linear system is $d-2$. In particular, $\operatorname{dimim}\left(c^{-E_{0}^{*}}\right)=d-2$.

For $k \geq 2$, we have to put together all the linear equations corresponding to all $D_{i}$. A block indexed by $t^{-d+2+j}$ and $|\mathbf{m}|=j^{\prime}$ will have $k$ rows. Again, all the blocks above the diagonal are zero. On the other hand, the rank of the diagonal block indexed by $t^{-d+2+j}$ and $|\mathbf{m}|=j$ is as large as possible, it is $\min \left\{k,\left(\begin{array}{c}j+2 \\ 2\end{array}\right)\right\}$. Indeed, its rows consists of the evaluation of $(u, v)$-monomials of degree $\leq j$ at points $p_{i}$ : since the points $p_{i}$ are generic they impose independent conditions on the corresponding (homogeneous) linear system (in variable $\left.\left(x_{1}, x_{2}, x_{3}\right)\right)$ of degree $j$. Hence, the rank of the matrix is $\sum_{j=0}^{d-3} \min \left\{k,\left(\begin{array}{c}j+2 \\ 2\end{array}\right)\right\}$. 
Theorem 11.2.1 For any $k \geq 1$ the dimension of $\operatorname{im}\left(c^{\left.-k E_{0}^{*}\right)}\right.$ is $\sum_{j=0}^{d-3} \min \left\{k,\left(\begin{array}{c}j+2 \\ 2\end{array}\right)\right\}$. The first value of $k$ when $c^{-k E_{0}^{*}}$ is dominant is $k=\left(\begin{array}{c}d-1 \\ 2\end{array}\right) . \operatorname{im}\left(c^{-k E_{0}^{*}}\right)$ has codimension 1 for $k=\left(\begin{array}{c}d-1 \\ 2\end{array}\right)-1$.

Accordingly, for a generic $\mathcal{L} \in \operatorname{im}\left(c^{-k E_{0}^{*}}\right), h^{1}\left(Z_{K}, \mathcal{L}\right)=p_{g}-\operatorname{dim}\left(\operatorname{im}\left(c^{-k E_{0}^{*}}\right)\right)$.

\subsection{The case $I^{\prime}=-k E_{0}^{*}(k \geq 1), Z=Z_{K}$ (and special divisor on $\mathrm{ECa}^{l^{\prime}}(Z)$ )}

In the previous subsection we considered generic points $\mathcal{P}:=\left\{p_{1}, \ldots, p_{k}\right\}$ on $C$, in particular, for all $j(0 \leq j \leq d-3)$ they imposed independent conditions on the linear system $\mathcal{O}_{\mathbb{P}^{2}}(j)$ (or, on the $(u, v)$-monomials of degree $\leq j$ ). However, taking special points they might fail to impose independent conditions on some $\mathcal{O}_{\mathbb{P}^{2}}(j)$. The discussion will show that $\operatorname{im}\left(c^{l^{\prime}}\right)$ has several (rather complicated) $h^{1}$-stratification, (some of them) imposed by special divisors.

Here we will indicate such possibilities; nevertheless, for simplicity we will restrict ourselves only to certain cases when only one block degenerates and the rang of the total linear system is determined again by the diagonal blocks. Even under this restriction we find the situation extremely rich, since it accumulates the classical plane curve geometry. However, the reader is invited to work out cases when the global rank depends on certain entries from the sub-diagonal blocks as well, covering even more sophisticated $h^{1}$-strata.

Recall that in the diagonal block of $\left(t^{-d+2+j},|\mathbf{m}|=j\right)$ we test if $\mathcal{P}$ impose independent conditions on $\mathcal{O}_{\mathbb{P}^{2}}(j)$ or not. In the sequel we will assume that there exits exactly one $j$, say $j_{0}$, when $\mathcal{P}$ fails to impose independent conditions. Clearly $j_{0}>0$. Furthermore, we will also assume that $\left(\begin{array}{c}j_{0}+1 \\ 2\end{array}\right) \leq k \leq\left(\begin{array}{c}j_{0}+3 \\ 2\end{array}\right)$. This means that in all the diagonal blocks with $j<j_{0}$ the number $k$ of rows is greater than or equal to the number $\left(\begin{array}{c}j+2 \\ 2\end{array}\right)$ of columns, hence the $j$-blocks has rank $\left(\begin{array}{c}j+2 \\ 2\end{array}\right)$. Symmetrically, in all the $j$-diagonal blocks with $j>j_{0}$ the number $k$ or rows is $\leq$ than the number $\left(\begin{array}{c}j+2 \\ 2\end{array}\right)$ of columns, hence the rank is $k$. Therefore, if the $j_{0}$-block is degenerated with rank $\min \left\{k,\left(\begin{array}{c}j_{0}+2 \\ 2\end{array}\right)\right\}-\Delta$ for some $\Delta>0$, then independently of the sub-diagonal entries, the rank of the matrix of the system is $\sum_{j=0}^{d-3} \min \left\{k,\left(\begin{array}{c}j+2 \\ 2\end{array}\right)\right\}-\Delta$. In particular, $h^{1}\left(Z_{K}, \mathcal{O}(D)\right)$ increases by $\Delta$ compared with the generic situation of 11.2.

Let us list some cases when such a degeneration can occur. Take e.g. $j_{0}=1$ and $k=3$ and $\left\{p_{1}, p_{2}, p_{3}\right\}$ are collinear. For $j_{0}=2$ we give two possibilities: either $k=4$ and the four points are collinear, or $k=6$ and the six points are contained in a conic.

We recall here two classical theorems of plane curve geometry, which can be used to produce similar examples; for more see the article [12] and the citations therein.

(a) [12, Prop. 1] For $j_{0} \geq 1$ and $k \leq 2 j_{0}+2$ the points $\mathcal{P}$ fail to impose independent conditions on $\mathcal{O}_{\mathbb{P} 2}\left(j_{0}\right)$ if and only if either $j_{0}+2$ points of $\mathcal{P}$ are collinear or $k=2 j_{0}+2$ and $\mathcal{P}$ is contained in a conic.

(b) [12, Th. Cayley-Bacharach4] Assume that $\mathcal{P}$ consists of $k=e \cdot f$ poinst which are the intersection points of two curves of degree $e$ and $f$. Then if a plane curve of degree $j_{0}=e+f-3$ contains all but one point of $\mathcal{P}$ then it contains all of $\mathcal{P}$. 


\section{Weighted homogeneous singularities}

\subsection{Preliminaries}

Assume that $(X, o)$ is a weighted homogeneous normal surface singularity, that is, there exists a normal affine variety $X^{a}$, which admits a good $\mathbb{C}^{*}$-action and singular point $o \in X^{a}$ such that $(X, o)$ is analytically isomorphic with $\left(X^{a}, o\right)$. This implies that the minimal good resolution graph $\Gamma$ is star shaped. As above, we assume that the link is a rational homology sphere, hence all the vertex-genera are zero. We write $v_{0}$ for the central vertex, hence $\Gamma \backslash v_{0}$ consists of $v$ strings. We assume that $v \geq 3$ (otherwise $p_{g}=0$, an uninteresting situation for the Abel map). Let $-b_{0}$ be the Euler number of $v_{0}$. The Euler numbers of the vertices $v_{j i}$ of the $j^{\text {th }}$ string $(1 \leq$ $j \leq v)$ are $-b_{j 1}, \ldots,-b_{j s_{j}}$, with $b_{j i} \geq 2$, determined by the continued fraction $\alpha_{j} / \omega_{j}=\left[b_{j 1}, \ldots, b_{j s_{j}}\right]$, where $\operatorname{gcd}\left(\alpha_{j}, \omega_{j}\right)=1,0<\omega_{j}<\alpha_{j}$. For each $j, v_{0}$ is connected with $v_{j 1}$ by one edge. The link is a Seifert fibered 3-manifold with Seifert invariants $\left(b_{0}, g=0 ;\left\{\left(\alpha_{j}, \omega_{j}\right)\right\}_{j}\right)$. In particular, the Seifert invariants characterize the topological type uniquely, see e.g. [41].

We denote by $E_{j i}$ the irreducible exceptional curves indexed by vertices $v_{j i}$. Let $P_{j}(1 \leq j \leq v)$ be $E_{v_{0}} \cap E_{j 1}$. One has the following result:

Theorem 12.1.1 (Analytic Classification Theorem) [9-11,42,54,55] The analytic isomorphism type of a normal surface weighted homogeneous singularity (with rational homology sphere link) with fixed Seifert invariants is determined by the analytic type of $\left(E_{v_{0}},\left\{P_{j}\right\}_{j}\right)$ modulo an action of $\operatorname{Aut}\left(E_{v_{0}},\left\{P_{j}\right\}_{j}\right)$. (This is the same as the analytic classification of Seifert line bundles over the projective line.)

Next we show that the minimal resolution of any weighted homogeneous singularity can be constructed by a special 'analytic plumbing'.

First we construct an analytic space $\widetilde{X^{a}}$ (the candidate for the resolution of $X^{a}$ ). Basically we mimic the analytic plumbing construction of the resolution of cyclic quotient singularities from e.g. [5,24]. Corresponding to the legs we fix distinct complex numbers $p_{j} \in \mathbb{C}$; the affine coordinates of the points $P_{j}$. Each leg, with divisors $\left\{E_{j i}\right\}_{i=1}^{s_{j}}, 1 \leq j \leq \nu$, will be covered by open sets $\left\{U_{j, i}\right\}_{i=0}^{s_{j}}$, copies of $\mathbb{C}^{2}$ with coordinates $\left(u_{j, i}, v_{j, i}\right)$. For each $1 \leq i \leq s_{j}$ we glue $U_{j, i-1} \backslash\left\{u_{j, i-1}=0\right\}$ with $U_{j, i} \backslash\left\{v_{j, i}=0\right\}$. The gluing maps are $v_{j, i}=u_{j, i-1}^{-1}\left(1 \leq i \leq s_{j}\right)$ and $u_{j, i}$ equals $u_{j, i-1}^{b_{j i}} v_{j, i-1}$ for $2 \leq i \leq s_{j}$ and $u_{j, 0}^{b_{j 1}}\left(v_{j, 0}-p_{j}\right)$ for $i=1$.

Furthermore, all $U_{j, 0}$ charts will be identified to each other: $u_{j, 0}=u_{k, 0}, v_{j, 0}=$ $v_{k, 0}$; denoted simply by $U_{0}$, with coordinates $\left(u_{0}, v_{0}\right)$. Till now, the curve $E_{v_{0}}$ appears only in $U_{0}$, it has equation $u_{0}=0$. To cover $E_{v_{0}}$ completely we need another copy $U_{-1}$ of $\mathbb{C}^{2}$ with coordinates $\left(u_{-1}, v_{-1}\right)$ as well; the gluing of $U_{0} \backslash\left\{v_{0}=0\right\}$ with $U_{-1} \backslash\left\{u_{-1}=0\right\}$ is $v_{0}=u_{-1}^{-1}, u_{0}=u_{-1}^{b_{0}} v_{-1}$. 


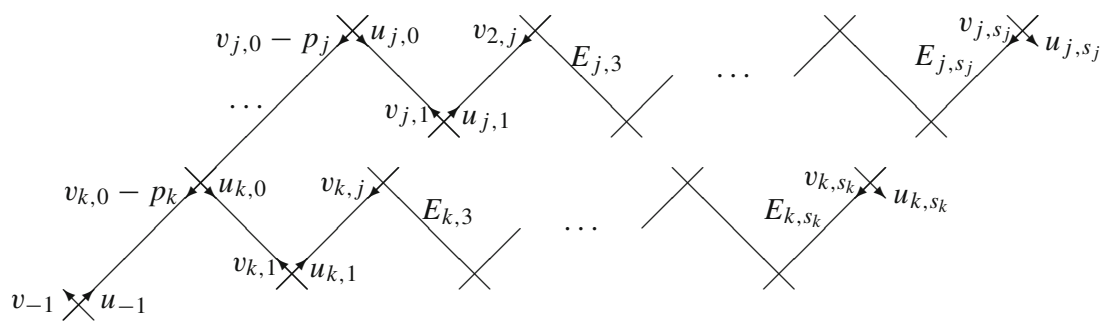

We call the output space $\widetilde{X^{a}}$. If we contract (analytically) $E=E_{v_{0}} \cup\left(\cup_{j, i} E_{j i}\right)$ we get a space $X^{a}$ whose germ at its singular point is a normal surface singularity $\left(X_{p l}, o\right)$. In this context, a resolution $\widetilde{X_{p l}}$ of $\left(X_{p l}, o\right.$ ) (as a subset of $\left.\widetilde{X^{a}}\right)$ is the pullback of a small Stein neighbourhood of $o$. The following statement is proved in [39]; basically it follows from the Analytic Classification Theorem 12.1.1 and from the fact that if we blow down the legs the obtained space carries naturally a Seifert line bundle structure over the projective line.

Proposition 12.1.2 The analytic structure on $\left(X_{p l}, o\right)$ carries a weighted homogeneous structure. Moreover, the minimal good resolution of any weighted homogeneous singularity with Seifert invariants $\left(b_{0}, g=0 ;\left\{\left(\alpha_{j}, \omega_{j}\right)\right\}_{j}\right)$ admits such an analytic plumbing representation for certain constants $\left\{p_{j}\right\}_{j}$ (that is, it can be embedded in some $\widetilde{X^{a}}$ constructed above via plumbing). By Theorem 12.1.1 we can even assume that each $p_{j}$ is non-zero (what we will assume below).

The $\mathbb{C}^{*}$ orbits lifted to $\widetilde{X^{a}}$ and closed are as follows: the generic ones, which intersect $E_{v_{0}}$ sit in $U_{0} \cup U_{-1}$ and are given by $\left\{v_{0}=c\right\}, c \in\left(\mathbb{C} \backslash\left\{\cup_{j}\left\{p_{j}\right\}\right\}\right) \cup \infty$. The special Seifert orbit for each $j$ in $U_{j, s_{j}}$ is given by $\left\{v_{j, s_{j}}=0\right\}$.

In the sequel we will identify our weighted homogeneous germ $(X, o)$ with such $\left(X_{p l}, 0\right)$. $\tau_{j}$.

For each $j$ we also introduce $0<\omega_{j}^{\prime}<\alpha_{j}$ such that $\omega_{j} \omega_{j}^{\prime}-1=\alpha_{j} \tau_{j}$ for some

\subsection{A basis for $H^{0}\left(\tilde{X} \backslash E, \Omega_{\tilde{X}}^{2}\right) / H^{0}\left(\tilde{X}, \Omega_{\tilde{X}}^{2}\right)$}

For $\ell,\left\{m_{j}\right\}_{j} \in \mathbb{Z}, n \in \mathbb{Z}_{\geq 0}$, let $\omega_{\ell, n}^{0}:=u_{0}^{-\ell-1} \prod_{j}\left(v_{0}-p_{j}\right)^{-m_{j}} v_{0}^{n} d v_{0} \wedge d u_{0}$ be a section of $\Omega_{\widetilde{X}}^{2}$ over $U_{0}$, with possible poles over $E \cap U_{0}$. This under the transformation $v_{0}=u_{-1}^{-1}, u_{0}=u_{-1}^{b_{0}} v_{-1}$ transforms into the following form on $U_{-1}$ :

$$
\pm u_{-1}^{-b_{0} \ell+\sum m_{j}-n-2} v_{-1}^{-\ell-1} \prod_{j}\left(1-u_{-1} p_{j}\right)^{-m_{j}} d u_{-1} \wedge d v_{-1}
$$

The regularity over $\widetilde{X} \backslash E$ requires that the exponent of $u_{-1}$ should be non-negative:

$$
n \leq-b_{0} \ell-2+\sum_{j} m_{j}
$$


Let is fix one of the legs, say $j$. By induction using substrings of the legs and the corresponding continued fraction identities (facts used intensively in cyclic quotient invariants computations) one gets that the transformation between chart $U_{0}$ and $U_{j, s_{j}}$ is $u_{0}=u_{j, s_{j}}^{-\tau_{j}} v_{j, s_{j}}^{-\omega_{j}}, v_{0}=u_{j, s_{j}}^{\omega_{j}^{\prime}} v_{j, s_{j}}^{\alpha_{j}}$. Then, $\omega_{\ell, n}^{0}$ in the chart $U_{j, s_{j}}$ under this transformation becomes

$$
\begin{gathered}
u_{j, s_{j}}^{\tau_{j} \ell-\omega_{j}^{\prime} m_{j}+\omega_{j}^{\prime}-1} v_{j, s_{j}}^{\omega_{j} \ell-\alpha_{j} m_{j}+\alpha_{j}-1}\left(u_{j, s_{j}}^{\omega_{j}^{\prime}} v_{j, s_{j}}^{\alpha_{j}}+p_{j}\right)^{n} \\
\cdot \prod_{j^{\prime} \neq j}\left(u_{j, s_{j}}^{\omega_{j}^{\prime}} v_{j, r_{j}}^{\alpha_{j}}+p_{j^{\prime}}-p_{j}\right)^{-m_{j}} d v_{j, s_{j}} \wedge d u_{j, s_{j}} .
\end{gathered}
$$

Again, by the regularity along $\widetilde{X} \backslash E$, the exponent of $v_{j, s_{j}}$ should be non-negative, hence $\omega_{j} \ell-\alpha_{j} m_{j}+\alpha_{j}-1 \geq 0$. The largest solution for $m_{j}$ is

$$
m_{j}=\left\lceil\omega_{j} \ell / \alpha_{j}\right\rceil
$$

Hence, the form $\omega_{\ell, n}^{0}$ extends to a form $\omega_{\ell, n}$ on $\tilde{X}$, regular on $\tilde{X} \backslash E$, if for $m_{j}:=\left\lceil\omega_{j} \ell / \alpha_{j}\right\rceil$ as in (12.2.2) (for all $j$ ) the inequality (12.2.1) holds. If $\ell<$ 0 then $m_{j}=\left\lceil\omega_{j} \ell / \alpha_{j}\right\rceil \leq 0$, hence the form $\omega_{\ell, n}$ is regular on $\tilde{X}$, and in $H^{0}\left(\tilde{X} \backslash E, \Omega_{\widetilde{X}}^{2}\right) / H^{0}\left(\widetilde{X}, \Omega_{\widetilde{X}}^{2}\right)$ it is zero. Hence, we can consider only the values $\ell \geq 0$. For them we set as a combination of the right hand side of (12.2.1) and (12.2.2)

$$
n_{\ell}:=-b_{0} \ell-2+\sum_{j}\left\lceil\omega_{j} \ell / \alpha_{j}\right\rceil \text {. }
$$

If $n_{\ell}<0$ then there is no such form with pole $\ell+1$ along $E_{v_{0}}$, cf. (12.2.1). Set $\mathcal{W}:=\left\{\ell \geq 0: n_{\ell} \geq 0\right\}$.

Lemma 12.2.3 [39] The forms $\omega_{\ell, n}\left(\ell \in \mathcal{W}, 0 \leq n \leq n_{\ell}\right)$ form a basis of $H^{0}\left(\widetilde{X} \backslash E, \Omega_{\widetilde{X}}^{2}\right) / H^{0}\left(\widetilde{X}, \Omega_{\widetilde{X}}^{2}\right)$.

Proof By their construction, the forms generate $H^{0}\left(\widetilde{X} \backslash E, \Omega_{\widetilde{X}}^{2}\right) / H^{0}\left(\widetilde{X}, \Omega_{\widetilde{X}}^{2}\right)$. But, by the $p_{g}$-formula of Pinkham [55], namely $p_{g}=\sum_{\ell \in \mathcal{W}}\left(n_{\ell}+1\right)$, their number is exactly $p_{g}$, the dimension of this quotient space.

Remark 12.2.4 $n_{0}=-2$, hence the $E_{v_{0}}$-pole of any $\omega_{\ell, n}$ is $\geq 2$.

\subsection{Natural line bundles}

Let $\widetilde{X^{a}}$ be as above, let $O^{a}$ be the closure of a lifted $\mathbb{C}^{*}$ orbit into $\widetilde{X^{a}}$, and set $O:=$ $O^{a} \cap \widetilde{X} \subset \widetilde{X}$.

Theorem 12.3.1 $\mathcal{O}_{\tilde{X}}(O)$ is a natural line bundle in $\operatorname{Pic}(\tilde{X})$.

Proof For each $O=O^{a} \cap \tilde{X}$ we find a local analytic function $f_{O}:(X, o) \rightarrow(\mathbb{C}, 0)$ such that the divisor in $\widetilde{X}$ of $f_{O} \circ \phi$ has the form $n_{O} O+\sum_{v} n_{v} E_{v}$ for some $n_{O}, n_{v} \in$ $\mathbb{Z}_{>0}$. This implies that $\mathcal{O}_{\widetilde{X}}\left(n_{O} O\right) \simeq \mathcal{O}_{\widetilde{X}}\left(-\sum_{v} n_{v} E_{v}\right)$. In order to find $f_{O}$ we use the 
fact that a weighted homogeneous germ is splice quotient $[45,46]$. In fact, by [43], the universal abelian cover (UAC) of $(X, o)$ is a Brieskorn complete intersection and certain powers of the coordinate functions of this complete intersection are the end curve functions of $(X, o)$ which have the wished properties for the orbits supported by the end vertices.

A similar argument clarifies the case of the other (generic) orbits as well. Let the UAC complete intersection equations be $\sum_{i, j} a_{i, j} z^{\alpha_{j}}=0,1 \leq j \leq v$, and $1 \leq i \leq v-2$, and where $\left\{a_{i, j}\right\}_{i, j}$ has full rank, cf. [43]. Then we add one more equation of type $\sum_{i} b_{i} z^{\alpha_{i}}+w=0$, such that the new larger matrix has again full rank. The new system corresponds to a splice quotient equations of the graph $\Gamma^{\prime}$ obtained from $\Gamma$ by blowing up the central vertex. The point is that the resolution with dual graph $\Gamma^{\prime}$ of this splice quotient singularity associated with $\Gamma^{\prime}$ can be obtained from $\widetilde{X}$ by blowing up a certain point $P \in E_{v_{0}} \backslash \cup_{j} P_{j} . P$ is determined by the choice of the coefficients $\left\{b_{i}\right\}_{i}$, and modification of the $\left\{b_{i}\right\}_{i}$ 's provides different points $P$. By the theory of splice quotient singularities, the end curve function $w=0$ cuts out an end curve in the UAC, which projected on $(X, o)$ is irreducible. Hence $\sum_{i} b_{i} z^{\alpha_{i}}$ is a weighted homogeneous function on the UAC of $(X, o)$, one of its powers is a homogeneous function on $(X, o)$ whose reduced zero set is irreducible. Its strict transform is some $O$, where $O \cap E_{v_{0}}$ depends on the choice of $\left\{b_{i}\right\}_{i}$. In particular all such orbits define the same line bundle, the natural line bundle.

\subsection{The Abel maps, $h^{1}(Z, \mathcal{L})$ and $\operatorname{dim} \operatorname{im}\left(d^{\prime}\right)$ for different line bundles and $I^{\prime}$}

In the sequel we fix a cycle $Z$ : for simplicity we assume that $Z \gg 0$, e.g. in the numerical Gorenstein case we can take $Z=Z_{K}$, or, in general, $Z \in Z_{K}+\mathcal{S}^{\prime}$ (in which cases for any $\mathcal{L} \in \operatorname{Pic}(\widetilde{X})$ with $c_{1}(\mathcal{L}) \in-\mathcal{S}^{\prime}$ one has $h^{1}(\widetilde{X}, \mathcal{L})=h^{1}\left(Z,\left.\mathcal{L}\right|_{Z}\right)$ ). In this case we will use all the differential forms $\omega_{\ell, n}$ constructed above. The interested reader might rewrite the statements and proofs below for smaller cycles (using forms a similar system of forms with poles $\leq Z$ ).

\subsection{The value $h^{1}\left(Z, \mathcal{O}_{Z}\left(-k E_{v_{0}}^{*}\right)\right), k \geq 1$}

Consider the natural line bundle $\mathcal{O}_{Z}\left(-E_{v_{0}}^{*}\right)$. If $O_{q}$ denotes the intersection of the generic $\mathbb{C}^{*}$-orbit with $\tilde{X}, O_{q} \cap E_{v_{0}}=\{q\}$ (where the intersection point $q$ can be identified with the $v_{0}$-affine coordinate in $\left.U_{0}\right)$, then by Theorem 12.3.1 $\mathcal{O}_{Z}\left(-E_{v_{0}}^{*}\right)=$ $\mathcal{O}_{Z}\left(O_{q}\right)$ for any $q \in E_{v_{0}} \backslash \cup_{j} E_{j, 1}$. Recall that $O_{q}$ in the chart $U_{0}$ is given by $\left\{v_{0}=q\right\}$. For $k$ distinct orbits $O_{q_{1}}, \ldots, O_{q_{k}}$ we apply Corollary 10.1.2. The restrictions are of type

$$
\sum_{n=0}^{n_{\ell}} a_{\ell, n} q_{i}^{n}=0, \text { for all } \ell \in \mathcal{W} \text { and } 1 \leq i \leq k
$$


Proposition 12.5.1 With the above notations, the number of independent relations (or, $p_{g}-h^{1}\left(Z, \mathcal{O}\left(-k E_{v_{0}}^{*}\right)\right)$, cf. Corollary 10.1.2) is $\sum_{\ell \in \mathcal{W}} \min \left\{n_{\ell}+1, k\right\}$. Hence

$$
h^{1}\left(Z, \mathcal{O}\left(-k E_{v_{0}}^{*}\right)\right)=\sum_{\ell \in \mathcal{W}} \max \left\{0, n_{\ell}+1-k\right\}
$$

Proof Use the previous discussion and $p_{g}=\sum_{\ell \in \mathcal{W}} n_{\ell}+1$.

E.g., for $k=1$ the number of independent relations is $\# \mathcal{W}$ and $h^{1}\left(Z, \mathcal{O}\left(-E_{v_{0}}^{*}\right)\right)=$ $p_{g}-\# \mathcal{W}$.

\subsection{The Zariski tangent space of $\operatorname{im}\left(d^{l^{\prime}}\right)$ at $\mathcal{O}_{Z}\left(I^{\prime}\right)$, for $I^{\prime}=-k E_{v_{0}}^{*}, k \geq 1$}

Take first $k=1, \mathcal{L}=\mathcal{O}_{Z}\left(l^{\prime}\right)=\mathcal{O}_{Z}\left(-E_{v_{0}}^{*}\right)$, and let $T_{\mathcal{L}} \operatorname{im}\left(c^{l^{\prime}}\right)$ be the Zariski tangent space of $\operatorname{im}\left(c^{l^{\prime}}\right)$ at $\mathcal{L}$. By Theorem 12.3.1 $O_{q} \in\left(c^{l^{\prime}}\right)^{-1}(\mathcal{L})$ for any $q \in E_{v_{0}} \backslash \cup_{j} P_{j}$, and (cf. Corollary 10.1.2 and 12.5) $\mathrm{im} T_{\mathcal{O}_{q}}\left(c^{l^{\prime}}\right)$ is the kernel of forms $\sum a_{\ell, n} \omega_{\ell, n}$ with $\sum_{n} a_{\ell, n} q^{n}=0$ for all $\ell \in \mathcal{W}$. We wish to describe the space generated by all subspaces $\operatorname{im} T_{\mathcal{O}_{q}}\left(c^{l^{\prime}}\right) \subset T_{\mathcal{L}} \operatorname{im}\left(c^{l^{\prime}}\right)$ when we move $q$. By taking $\left(n_{\ell}+1\right)$ different values $q_{r}$ we get that the vectors $\left(q_{r}^{0}, q_{r}^{1}, \ldots, q^{n_{\ell}}\right)$ (dual to the hyperplane $\sum_{n} a_{\ell, n} q_{r}^{n}=0$ ) are linearly independent (since their Vandermonde determinant is non-zero), hence $\sum_{q} \operatorname{im} T_{\mathcal{O}_{q}}\left(c^{l^{\prime}}\right)=T_{\mathcal{L}} \mathrm{Pic}^{l^{\prime}}(Z)$, the whole tangent space of $\operatorname{Pic}^{l^{\prime}}(Z)$ at $\mathcal{L}$. Hence we proved the following statement for $k=1$.

Theorem 12.6.1 $T_{\mathcal{L}} \operatorname{im}\left(c^{l^{\prime}}\right)=T_{\mathcal{L}} \mathrm{Pic}^{l^{\prime}}(Z)$ for any $l^{\prime}=-k E_{v_{0}}^{*}, k \geq 1$.

The general case $k \geq 1$ follows from the case $k=1$ and (6.1.2).

\subsection{The value $h^{1}\left(Z, \mathcal{O}_{Z}\left(-E_{j, s_{j}}^{*}\right)\right)$}

Fix some leg $j$ and consider the corresponding end-vertex $E_{j, s_{j}}$ and the natural line bundle $\mathcal{O}_{Z}\left(-E_{j, s_{j}}^{*}\right)$. If $O_{j}$ denotes the intersection of the special $\mathbb{C}^{*}$-orbit with $\widetilde{X}$, then in $U_{j, s_{j}}$ it is given by $\left\{v_{j, s_{j}}=0\right\}$ and by Theorem 12.3.1 $\mathcal{O}_{Z}\left(-E_{j, s_{j}}^{*}\right)=\mathcal{O}_{Z}\left(O_{j}\right)$. We apply again Corollary 10.1.2 for the forms $\omega_{\ell, n}$ in $U_{j, s_{j}}$ (cf. 12.2)

$$
\begin{gathered}
u_{j, s_{j}}^{\tau_{j} \ell-\omega_{j}^{\prime} m_{j}+\omega_{j}^{\prime}-1} v_{j, s_{j}}^{\omega_{j} \ell-\alpha_{j} m_{j}+\alpha_{j}-1}\left(u_{j, s_{j}}^{\omega_{j}^{\prime}} v_{j, s_{j}}^{\alpha_{j}}+p_{j}\right)^{n} \\
\cdot \prod_{j^{\prime} \neq j}\left(u_{j, s_{j}}^{\omega_{j}^{\prime}} v_{j, r_{j}}^{\alpha_{j}}+p_{j^{\prime}}-p_{j}\right)^{-m_{j}} d v_{j, s_{j}} \wedge d u_{j, s_{j}} .
\end{gathered}
$$

Some of the forms have no poles along $\left\{u_{j, s_{j}}=0\right\}$, hence they determine no restrictions. That is, any restriction appears only if $\tau_{j} \ell-\omega_{j}^{\prime} m_{j}+\omega_{j}^{\prime}-1<0$.

We recall that the $v_{j, s_{j}}$ exponent $\omega_{j} \ell-\alpha_{j} m_{j}+\alpha_{j}-1$ is non-negative. However, if this exponent is strict positive, then the restriction to $\left\{v_{j, s_{j}}=0\right\}$ is zero. Hence, restriction appears only if this exponent is exactly zero. Note that $\omega_{j} \ell-\alpha_{j} m_{j}+\alpha_{j}-$ $1=0$ if and only if $\alpha_{j} \mid \omega_{j} \ell-1$. 
Proposition 12.7.1 The number of independent relations, $p_{g}-h^{1}\left(Z, \mathcal{O}_{Z}\left(-E_{j, s_{j}}^{*}\right)\right)$, is

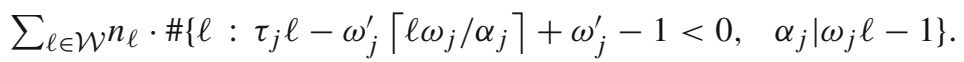

\subsection{The dimension of im $\left(c^{\prime}\right)$ for $l^{\prime}=-E_{v_{0}}^{*}$}

Let $d:=\left(Z, l^{\prime}\right)$, this is the dimension of $\mathrm{ECa}^{l^{\prime}}(Z)$ (cf. Theorem 3.1.10). In fact, $\mathrm{ECa}^{l^{\prime}}(Z)$ projects onto $E_{v_{0}} \backslash \cup_{j} P_{j}$ with fibers $\simeq \mathbb{C}^{d-1}$. We are again in chart $U_{0}$, and we simplify the coordinate notations $\left(u_{0}, v_{0}\right)$ into $(u, v)$. We have to restrict the forms $\omega_{\ell, n}$ to the generic transversal cut $\widetilde{D}_{g e n}$ given by $\left\{v=c_{0}+c_{1} u+\cdots+c_{d-1} u^{d-1}\right\}$. In this generic case the linear system is more complicated, the rank is much harder to compute.

Recall $p_{g}=\sum_{\ell \in \mathcal{W}}\left(n_{\ell}+1\right)$. We define the function $s: \mathbb{Z}_{\geq 0} \rightarrow \mathbb{Z}_{\geq 0}$ by decreasing induction. First, set $s(\ell)=0$ for any $\ell \gg 0$ (e.g. for any $\ell$ larger than any element of $\mathcal{W})$. Then define

$$
s(\ell):= \begin{cases}\max \{0, s(\ell+1)-1\} & \text { if } \ell \notin \mathcal{W} \\ s(\ell+1)+n_{\ell} & \text { if } \ell \in \mathcal{W}\end{cases}
$$

Lemma 12.8.2 (a) $s(0) \leq p_{g}-\# \mathcal{W}$.

(b) The following facts are equivalent: $(i) s(0)=p_{g}-\# \mathcal{W}$, (ii) $s(\ell)=0$ for all $\ell \geq 0$, (iii) $n_{\ell}=0$ for all $\ell \in \mathcal{W}$, (iv) $p_{g}=\# \mathcal{W}$ and $s(0)=0$.

Proof (a) By a decreasing induction one gets

$$
s(\ell)=\sum_{\ell^{\prime} \geq \ell, \ell \in \mathcal{W}} n_{\ell^{\prime}}-\#\left\{\ell^{\prime}: \ell^{\prime} \geq \ell, \ell^{\prime} \notin \mathcal{W}, s\left(\ell^{\prime}+1\right)>0\right\} .
$$

In particular,

$$
s(0)=p_{g}-\# \mathcal{W}-\#\{\ell: \ell \geq 0, \ell \notin \mathcal{W}, s(\ell+1)>0\} \leq p_{g}-\# \mathcal{W} .
$$

(b) (ii) $\Rightarrow($ iii $) \Rightarrow($ iv $) \Rightarrow($ i $)$ are easy, we prove $(i) \Rightarrow($ ii $)$. We use from (12.8.3) that $(\dagger)$ $\{\ell \geq 0, \ell \notin \mathcal{W}, s(\ell+1)>0\}=\emptyset$. Recall that $0 \notin \mathcal{W}$ (cf. 12.2.4). Hence $s(1)=0$ (and necessarily $s(0)=0$ too). If $1 \in \mathcal{W}$ then $s(2)=0$ from the definition of $s(1)$, if $1 \notin \mathcal{W}$ then $s(2)=0$ from $(\dagger)$. Repeating the argument, we get (ii).

Theorem 12.8.4 $h^{1}\left(Z, \mathcal{O}_{Z}\left(\widetilde{D}_{\text {gen }}\right)\right)=s(0)$, hence

$$
\text { number of independent relations }=\operatorname{dim} \operatorname{im}\left(c^{l^{\prime}}\right)=p_{g}-s(0) .
$$

Recall that if $O_{\text {gen }}$ is the intersection of the generic $\mathbb{C}^{*}$-orbit with $\tilde{X}$, then the natural line bundle $\mathcal{O}_{Z}\left(-E_{v_{0}}^{*}\right)$ is $\mathcal{O}_{Z}\left(O_{g e n}\right)$ and $h^{1}\left(Z, \mathcal{O}_{Z}\left(O_{g e n}\right)\right)=p_{g}-\# \mathcal{W}$. This identity 
and the one from Theorem 12.8.4 show that the inequality from (12.8.3) is compatible with the semicontinuity of $h^{1}$, cf. 5.2.1.

Corollary 12.8.5 $h^{1}\left(Z, \mathcal{O}_{Z}\left(\widetilde{D}_{g e n}\right)\right)=h^{1}\left(Z, \mathcal{O}_{Z}\left(O_{\text {gen }}\right)\right)$ if and only if $n_{\ell}=0$ for all $\ell \in \mathcal{W}$, and in this case in fact $h^{1}\left(Z, \mathcal{O}_{Z}\left(O_{g e n}\right)\right)=0$ and $c^{-E_{v_{0}}^{*}}$ is dominant.

In the next proof we write $\mathcal{W}=\left\{\ell_{1}, \ldots, \ell_{\max }\right\}$ with $\ell_{1}<\cdots<\ell_{\max }$.

12.8.6 Proof of Theorem 12.8.4 According to Corollary 10.1.2 we have to find the dimension

$$
\left\{\left\{a_{\ell, n}\right\}_{\ell \in \mathcal{W}, 0 \leq n \leq n_{\ell}}: \operatorname{Res}_{\widetilde{D}_{g e n}}\left(\sum_{\ell, n} a_{\ell, n} \omega_{\ell, n}\right) \text { has no pole along } \widetilde{D}_{g e n}\right\}
$$

Recall that $\widetilde{D}_{g e n}=\left\{v=c_{0}+c_{1} u+\cdots+c_{d-1} u^{d-1}\right\}$ with $c_{i}$ generic. In fact, what we will need is $c_{1} \neq 0$. If we set $v^{\prime}:=v-\left(c_{0}+c_{1} u+\cdots+c_{d-1} u^{d-1}\right)$ then $d v \wedge d u=d v^{\prime} \wedge d u$, hence in the form $d v \wedge d u$ can be replaced by $d v^{\prime} \wedge d u$, and $\operatorname{Res}_{\widetilde{D}_{g e n}}\left(\sum_{\ell, n} a_{\ell, n} \omega_{\ell, n}\right)=\left.\left(\sum_{\ell, n} a_{\ell, n} \omega_{\ell, n} / d v\right)\right|_{\widetilde{D}_{g e n}}$.

The vanishing of poles provides a linear system whose matrix will be labelled as follows. The columns are indexed by $\{\ell, n\}_{\ell \in \mathcal{W}, n}$. We subdivide this in vertical blocks. The first one, $B\left(\ell_{1}\right)$, contains $n_{\ell_{1}}+1$ columns, the second one, $B\left(\ell_{2}\right)$, contains $n_{\ell_{2}}+1$ columns, etc. Their columns are indexed by the corresponding $n$ 's. The rows are indexed by the pole orders: the first row corresponds to $u^{-\ell_{\max }-1}$, the last one to $u^{-1}$.

We define also the sub-block $B^{\prime}\left(\ell_{i}\right)\left(\ell_{i} \in \mathcal{W}\right)$ of $B\left(\ell_{i}\right)$, it is obtained from $B\left(\ell_{i}\right)$ by deleting the rows corresponding to pole orders strict higher than $\ell_{i}+1$ (rows $u^{-\ell-1}$, $l>l_{i}$ ). In fact, all the entries of the deleted part are zero, and the highest row kept in $B^{\prime}\left(\ell_{i}\right)$ contains non-zero entries.

We proceed by induction: we fix some $\ell, 0 \leq \ell \leq \ell_{\text {max }}$, and assume that $\ell_{i-1}<$ $\ell \leq \ell_{i}$ for some $\ell_{i} \in \mathcal{W}$. Then we consider the submatrix $M(\ell)$ (in the up-right corner) containing all the entries from the columns contained in the vertical blocks $B\left(\ell_{j}\right)$ with $\ell_{j} \geq \ell_{i}$, and from the rows $u^{-l^{\prime}-1}$ with $l \leq l^{\prime} \leq l_{\text {max }}$. It is the matrix of a linear system with $l_{\text {max }}-\ell+1$ equations and with variables $\left\{\left\{a_{\ell_{j}, n}\right\}_{\ell_{j} \in \mathcal{W}, \ell_{j} \geq \ell_{i}, 0 \leq n \leq n_{\ell_{j}}}\right.$, what we formulate next. By decreasing induction we prove that $s(\ell)$ is exactly the dimension $\operatorname{dim}(\ell)$ of

$\left\{\left\{a_{\ell_{j}, n}\right\}_{\ell_{j} \geq \ell_{i}, 0 \leq n \leq n_{\ell_{j}}}:\left.\left(\sum_{\ell_{j} \geq \ell_{i}, n} a_{\ell_{j}, n} \omega_{\ell_{j}, n} / d v\right)\right|_{\widetilde{D}_{g e n}}\right.$ has no pole of order $\left.\geq \ell+1\right\}$.

If $\ell=\ell_{\max }$, then the system contains $n_{\ell_{\max }}+1$ variables and a nontrivial equation (one checks that at least one entry of the system is non-zero), hence $\operatorname{dim}\left(\ell_{\max }\right)=$ $n_{\ell_{\max }}=s\left(\ell_{\max }\right)$.

When we step from $\ell+1$ to $\ell\left(0 \leq \ell<\ell_{\max }\right)$, we have to consider two cases.

First assume that $\ell \in \mathcal{W}$ ( say $\ell=\ell_{i}$ ). Then we add $n_{\ell}+1$ new variables and one new equation. In the columns corresponding to the new variables only the last row contains non-zero entries, but this part indeed contains at least one non-zero entry. Hence the new equation is linearly independent from the old ones, and $\operatorname{dim}(l)=$ $\operatorname{dim}(l+1)+n_{\ell}+1-1$; this is the inductive step for $s(\ell)$ too. 
If $\ell \notin \mathcal{W}$, say $\ell_{i-1}<\ell<\ell_{i}$, then in the new system one has the same number of variables, but there is one more equation corresponding to the new row $\ell$. We divide this case into two subcases. First, assume that the rank of $M(\ell+1)$ equals the number of columns $\sum_{\ell_{j} \geq \ell_{i}}\left(n_{\ell_{j}}+1\right)$. Hence, adding a new row we cannot increase the rank, hence $\operatorname{dim}(\ell+1)=\operatorname{dim}(\ell)$. In fact, $\operatorname{dim}(\ell+1)=0$, and the new equation (even if it is 'generic') cannot decrease the dimension of the system.

In the second case we assume that the rank of $M(\ell+1)$ is strict smaller than the number of columns $\sum_{\ell_{j} \geq \ell_{i}}\left(n_{\ell_{j}}+1\right)$. In this case we claim $\operatorname{rank}(M(\ell))=\operatorname{rank}(M(\ell+$ $1)+1)$, hence $\operatorname{dim}(\ell)=\operatorname{dim}(\ell+1)-1$. This again agrees with (12.8.1). The claim follows from the next lemma via standard linear algebra.

Lemma 12.8.7 Fix $\ell_{i} \in \mathcal{W}$. Assume that the hight $\ell_{i}+1$ of the sub-block $B^{\prime}\left(\ell_{i}\right)$ is not smaller then its width $n_{\ell_{i}}+1$. Then the top $\left(n_{\ell_{i}}+1\right) \times\left(n_{\ell_{i}}+1\right)$ minor $M^{\prime}$ of $B^{\prime}\left(\ell_{i}\right)$ has non-zero determinant. Furthermore, if the hight $\ell_{i}+1$ of the sub-block $B^{\prime}\left(\ell_{i}\right)$ is smaller then its width $n_{\ell_{i}}+1$ then the $\ell_{i}+1$ rows of $B^{\prime}\left(\ell_{i}\right)$ are linearly independent.

We prove this lemma in two steps. The first step is the next statement.

Lemma 12.8.8 For any $m \in \mathbb{Z}_{>0}$ we construct the $m \times m$-matrix $M(c)$ as follows. Its $n^{\text {th }}$-column consists of the first $m$ coefficients of the series $\left(\sum_{k \geq 0} c_{k} u^{k}\right)^{n-1}$. E.g., the first column has entries $(1,0, \ldots)$, the second one $\left(c_{0}, c_{1}, \ldots\right)$, the third one $\left(c_{0}^{2}, 2 c_{0} c_{1}, \ldots\right)$. Then $\operatorname{det} M(c)=c_{1}^{m(m-1) / 2}$.

Proof Assume that $\left\{C_{n}\right\}_{1 \leq n \leq m}$ are the columns of $M(c)$. Consider the matrix $M(c)^{\prime}$ consisting of columns $\left\{C_{n}^{\prime}\right\}_{1 \leq n \leq m}, C_{1}^{\prime}=C_{1}, C_{2}^{\prime}=C_{2}-c_{0} C_{1}, C_{3}^{\prime}=C_{3}-2 c_{0} C_{2}+$ $c_{0}^{2} C_{1}$, etc. Then $\operatorname{det} M(c)=\operatorname{det} M(c)^{\prime}$. But $C_{n}^{\prime}$ consists of the coefficients of $\left(-c_{0}+\right.$ $\left.\sum_{k \geq 0} c_{k} u^{k}\right)^{n-1}$, hence the entries of $M(c)^{\prime}$ above the diagonal are zero, and on the diagonal one has the entries $1, c_{1}, c_{1}^{2}, \ldots$

Finally we prove Lemma 12.8.8. We apply Lemma 12.8 .7 for $m=\ell_{i}+1$. Note that we can consider only the first situation (when the height is sufficiently large, since in the other case the matrix can be completed to a square matrix of size $n_{\ell_{i}}+1$ with non-zero determinant.

For fixed $\ell_{i}$ the forms have the form $\omega_{\ell_{i}, n}=u^{-\ell_{i}-1} f_{\ell_{i}}\left(v_{0}\right) v_{0}^{n} d v_{0} \wedge d u_{0}, 0 \leq n \leq$ $n_{\ell_{i}}$, where $f_{\ell_{i}}\left(v_{0}\right):=\prod_{j}\left(v_{0}-p_{j}\right)^{-m_{j}}$. Let us we substitute $v_{0} \mapsto \sum_{k \geq 0} c_{k} u^{k}$ in this function, and consider its Taylor expansion $P(u)=b_{0}+b_{1} u+\cdots$. Then the columns of the top minor $M^{\prime}$ of $B^{\prime}\left(\ell_{i}\right)$ are the coefficients of the product $\left(\sum_{k \geq 0} c_{k} u^{k}\right)^{n} \cdot P(u)$. Since $b_{0} \neq 0$, this means that $M^{\prime}$ is obtained from $M(c)$ by multiplication with an invertible matrix. Hence det $M^{\prime} \neq 0$.

This ends the proof of Theorem 12.8.4.

\subsection{The Abel map $c^{\prime}$ in a neighborhood of some $0_{q}$ supported by $E_{v_{0}}$}

Since we have a basis of differential forms, using the results and the notations of Sect. 7.3 we are able to give the 'complete Abel map'. Indeed, assume that $O$ is the intersection of a generic $\mathbb{C}^{*}$-orbit with $\widetilde{X}$, and in some local chart it is given by 
$v=0$. Consider the parametrization of its neighbourhood in $\mathrm{ECa}^{l^{\prime}}(Z)$ in the form $D(c)=\left\{v=c_{0}+c_{1} u+\cdots+c_{d-1} u^{d-1}\right\}\left(\left|c_{0}\right| \ll 1\right)$, where $d=\left(Z, l^{\prime}\right)$ is the dimension of $\mathrm{ECa}^{l^{\prime}}(Z)$. Above we constructed $p_{g}$ differential forms having in this chart the expressions $\omega_{\ell, n}^{0}=u^{-\ell-1} f_{\ell, n} v^{n} d v \wedge d u$, where $f_{\ell, n}:=\prod_{j}\left(v-p_{j}\right)^{-m_{j}}, \ell \in \mathcal{W}$ and $0 \leq n \leq n_{\ell}$. Then the Abel map restricted to this chart is $c \mapsto\left(\left\langle\left\langle D(c), \omega_{\ell, n}\right\rangle\right\rangle\right)_{\ell, n}$, where each coordinate $\left\langle\left\langle D(c), \omega_{\ell, n}\right\rangle\right\rangle$ is determined explicitly in (7.3.5).

The reader is invited to take his/her favorite star-shaped graph, determine explicitly the forms and the corresponding Abel map. Here we will exemplify the general description by an example when the image of the Abel map is a singular hypersurface.

Example 12.9.1 Consider the star-shaped graph with $b_{0}=4, v=8,\left(\alpha_{j}, \omega_{j}\right)=(8,1)$ for all $j$. Then $p_{g}=3$. By a computation $\mathcal{W}=\{1\}$, and the three forms are $u^{-2} f(v) v^{n}$, where $n=0,1,2$, and $f(v)=\prod_{j}\left(v-p_{j}\right)^{-1}$. By a computation (using Laufer's algorithm) $Z_{\text {min }}=E+E_{v_{0}}$, and $h^{1}\left(\mathcal{O}_{Z_{\text {min }}}\right)=3$. In fact, $\left\lfloor Z_{K}\right\rfloor=Z_{\text {min }}$. We determine the Abel map $c^{l^{\prime}}$ for $Z=Z_{\text {min }}$ and $l^{\prime}=-E_{v_{0}}^{*}$. Hence $d=\left(Z, l^{\prime}\right)=2$, and $D(c)=\left\{v=c_{0}+c_{1} u\right\}$. By (7.3.5) the Abel map is

$$
\left(c_{0}, c_{1}\right) \mapsto\left(-c_{1} f\left(c_{0}\right),-c_{1} c_{0} f\left(c_{0}\right),-c_{1} c_{0}^{2} f\left(c_{0}\right)\right) .
$$

If $(A, B, C)$ are the coordinates in the target, then $\operatorname{im}(c)=\left\{A C=B^{2}\right\}$. It is surprising that $\operatorname{im}(c)$ is independent of the choice of the points $\left\{p_{j}\right\}_{j}$ (that is, of the analytic structure of $(X, o))$.

Acknowledgements Open access funding provided by MTA Alfréd Rényi Institute of Mathematics (MTA RAMKI). We thank the referee for the careful reading of the manuscript.

Open Access This article is distributed under the terms of the Creative Commons Attribution 4.0 International License (http://creativecommons.org/licenses/by/4.0/), which permits unrestricted use, distribution, and reproduction in any medium, provided you give appropriate credit to the original author(s) and the source, provide a link to the Creative Commons license, and indicate if changes were made.

\section{References}

1. Arbarello, E., Cornalba, M., Griffiths, P.A., Harris, J.: Geometry of algebraic curves, Grundlehren der Mathematischen Wissenschaften 267, vol. I. Springer, New York (1985)

2. Artin, M.: Some numerical criteria for contractibility of curves on algebraic surfaces. Am. J. Math. 84, 485-496 (1962)

3. Artin, M.: On isolated rational singularities of surfaces. Am. J. Math. 88, 129-136 (1966)

4. Artin, M.: Algebraic approximation of structures over complete local rings. Publ. Math. de l'IHES 36, 23-58 (1969)

5. Barth, W., Peters, C., Van de Ven, A.: Compact Complex Surfaces, Ergebnisse der Mathematik und ihrer Grenzgebiete, 3 Folge, Band. A Series of Modern Surveys in Mathematics, vol. 4. Springer, Berlin (1984)

6. Braun, G., Némethi, A.: Surgery formula for Seiberg-Witten invariants of negative definite plumbed 3-manifolds. J. für die reine und ang. Math. 638, 189-208 (2010)

7. Campillo, A., Delgado, F., Gusein-Zade, S.M.: Poincaré series of a rational surface singularity. Invent. Math. 155(1), 41-53 (2004)

8. Campillo, A., Delgado, F., Gusein-Zade, S.M.: Universal abelian covers of rational surface singularities and multi-index filtrations. Funk. Anal. i Prilozhen. 42(2), 3-10 (2008)

9. Conner, P.E., Raymond, F.: Holomorphic seifert fibrations (Proc. Conf. Transformation Groups, Amherst, 1973). Lect. Notes Math. 299, 124-204 (1974) 
10. Dolgachev, I.: Automorphic forms and quasihomogeneous singularities. Func. Anal. Appl. 9, 149-151 (1975)

11. Dolgachev, I.: Automorphic forms and weighted homogeneous equations (1977) (Unpublished typed manuscript)

12. Eisenbud, D., Green, M., Harris, J.: Cayley-Bacharach theorems and conjectures. Bull. AMS 33(3), 295-324 (1996)

13. Flamini, F.: Lectures on Brill-Noether theory. In: Muk, J.M., Kim, Y.R. (eds.) Proceedings of the workshop "Curves and Jacobians", pp. 1-20. Korea Institute for Advanced Study, Seoul (2011)

14. Grauert, H., Remmert, R.: Coherent analytic sheaves, Grundlehren der mathematischen Wissenschaften, vol. 265. Springer, Berlin (1984)

15. Grauert, H., Riemenschneider, O.: Verschwindungssätze für analytische kohomologiegruppen auf komplexen Räumen. Invent. Math. 11, 263-292 (1970)

16. Grothendieck, A.: Fondements de la géométrie algébrique, [Extraits du Séminaire Bourbaki 19571962]. Secrétariat mathématique, Paris (1962)

17. Hartshorne, R.: Algebraic Geometry, Graduate Texts in Mathematics, vol. 52. Springer, Berlin (1977)

18. Hironaka, H.: On the equivalence of singularities. I, Arithmetical Algebraic Geometry (Proc. Conf. Purdue Univ.), pp. 153-200. Harper \& Row, New York (1963)

19. Kleiman, StL: The Picard scheme, in 'Fundamental Algebraic Geometry: Grothendieck's FGA Explained'. Math. Surv. Monogr. 123, 248-333 (2005)

20. Kleiman, S.L.: The Picard Scheme. In: Schneps, L. (ed.) Alexandre Grothendieck: A Mathematical Portrait. International Press of Boston Inc, Cambridge (2014)

21. Kollár, J., Némethi, A.: Durfee's conjecture on the signature of smoothings of surface singularities, (an appendix by T. de Fernex). J. Ann. Sci. de l'Ecole Norm. Sup. 50(3), 787-798 (2017)

22. László, T.: Lattice cohomology and Seiberg-Witten invariants of normal surface singularities, Ph.D. thesis, Central European University, Budapest (2013)

23. László, T., Nagy, J., Némethi, A.: Surgery formulae for the Seiberg-Witten invariant of plumbed 3manifolds. Rev. Mat. Complut. (2019). https://doi.org/10.1007/s13163-019-00297-z

24. Laufer, H.B.: Normal Two-dimensional Singularities. Annals of Math. Studies, vol. 71. Princeton University Press, Princeton (1971)

25. Laufer, H.B.: On rational singularities. Am. J. Math. 94, 597-608 (1972)

26. Laufer, H.B.: On minimally elliptic singularities. Am. J. Math. 99, 1257-1295 (1977)

27. Lipman, J.: Rational singularities, with applications to algebraic surfaces and unique factorization. Inst. Hautes Études Sci. Publ. Math. 36, 195-279 (1969)

28. Luengo, I.: The $\mu$-constant stratum is not smooth. Invent. Math. 90(1), 139-152 (1987)

29. Luengo, I., Melle-Hernández, A., Némethi, A.: Links and analytic invariants of superisolated singularities. J. Algebraic Geom. 14, 543-565 (2005)

30. Macdonald, I.G.: Symmetric Functions and Hall polynomials, Oxford Math. Monographs. Clarendon Press, Oxford (1995)

31. Mumford, D.: Lectures on curves on an algebraic surface, Ann Studies, vol. 59. Princeton University Press, Princeton (1966)

32. Nagy, J., Némethi, A.: The Abel map for surface singularities II. Generic analytic structure; arXiv (2018)

33. Némethi, A.: "Weakly" Elliptic Gorenstein singularities of surfaces. Invent. Math. 137, 145-167 (1999)

34. Némethi, A.: Five lectures on normal surface singularities, lectures at the Summer School in Low dimensional topology Budapest, Hungary, 1998. Bolyai Soc. Math. Stud. 8, 269-351 (1999)

35. Némethi, A.: Graded Roots and Singularities, Singularities in Geometry and Topology, pp. 394-463. World Scientific Publishing, Hackensack (2007)

36. Némethi, A.: Poincaré series associated with surface singularities. In: Singularities I, 271-297, Contemp. Math. 474, Amer. Math. Soc., Providence RI (2008)

37. Némethi, A.: The cohomology of line bundles of splice-quotient singularities. Adv. Math. 229(4), 2503-2524 (2012)

38. Némethi, A.: The Seiberg-Witten invariants of negative definite plumbed 3-manifolds. J. Eur. Math. Soc. 13, 959-974 (2011)

39. Némethi, A.: Normal surface singularities (book in preparation)

40. Némethi, A., Okuma, T.: Analytic singularities supported by a specific integral homology sphere link. Methods Appl. Anal. 24(2), 303-320 (2017) 
41. Neumann, W.D.: A calculus for plumbing applied to the topology of complex surface singularities and degenerating complex curves. Trans. Am. Math. Soc. 268(2), 299-344 (1981)

42. Neumann, W.D.: Geometry of quasihomogeneous surface singularities, Singularities, Part 2 (Arcata, Calif., 1981). In: Proc. Sympos. Pure Math., 40, pp. 245-258, Amer. Math. Soc., Providence, RI (1983)

43. Neumann, W.D.: Abelian covers of quasihomogeneous surface singularities, Singularities, Part 2 (Arcata, Calif., 1981). In: Proc. Sympos. Pure Math., 40, pp. 233-243. Amer. Math. Soc., Providence, RI (1983)

44. Neumann, W., Wahl, J.: Casson invariants of links of singularities. Comment. Math. Helvetici 65, 58-78 (1990)

45. Neumann, W., Wahl, J.: Complete intersection singularities of splice type as universal abelian covers. Geom. Topol. 9, 699-755 (2005)

46. Neumann, W., Wahl, J.: Complex surface singularities with integral homology sphere links. Geom. Topol. 9, 757-811 (2005)

47. Neumann, W.D., Wahl, J.: The End Curve Theorem for normal complex surface singularities. J. Eur. Math. Soc. 12, 471-503 (2010)

48. Okuma, T.: Universal abelian covers of rational surface singularities. J. Lond. Math. Soc. 70(2), 307324 (2004)

49. Okuma, T.: The geometric genus of splice-quotient singularities. Trans. Am. Math. Soc. 360(12), $6643-6659$ (2008)

50. Okuma, T.: Another proof of the end curve theorem for normal surface singularities. J. Math. Soc. Jpn. 62(1), 1-11 (2010)

51. Okuma, T., Watanabe, K., Yoshida, K.: Good ideals and $p_{g}$-ideals in two-dimensional normal singularities. arXiv: 1407.1590

52. Okuma, T., Watanabe, K., Yoshida, K.: Rees algebras and $p_{g}$-ideals in a two-dimensional normal local domain. arXiv: 1511.00827

53. Okuma, T., Watanabe, K., Yoshida, K.: A characterization of two-dimensional rational singularities via core of ideals. J. Algebra 499, 450-468 (2018)

54. Orlik, P., Wagreich, Ph: Algebraic surfaces with $k^{*}$-action. Acta Math. 138, 45-81 (1977)

55. Pinkham, H.: Normal surface singularities with $\mathbb{C} *$ action. Math. Ann. 227, 183-193 (1977)

56. Ramanujam, C.P.: Remarks on Kodaira vanishing theorem. J. Indian Math. Soc. 36, 41-51 (1972)

57. Reid, M.: Chapters on Algebraic Surfaces. In: Kollár, J. (ed.) Complex Algebraic Geometry, Mathematical Series, vol. 3, pp. 3-159. IAS, Park City (1997)

58. Yau, S.S.-T.: On strongly elliptic singularities. Am. J. Math. 101, 855-884 (1979)

59. Yau, S.S.-T.: On maximally elliptic singularities. Trans. AMS 257(2), 269-329 (1980)

Publisher's Note Springer Nature remains neutral with regard to jurisdictional claims in published maps and institutional affiliations.

\section{Affiliations}

\section{János Nagy ${ }^{1}$ - András Némethi' ${ }^{2,3,4}$}

\ András Némethi

nemethi.andras@renyi.mta.hu

János Nagy

nagy_janos@phd.ceu.edu

1 Department of Mathematics, Central European University, Budapest, Hungary

2 Alfréd Rényi Institute of Mathematics, Hungarian Academy of Sciences, Reáltanoda utca 13-15, Budapest 1053, Hungary

3 Department of Geometry, ELTE-University of Budapest, Budapest, Hungary

4 BCAM-Basque Center for Applied Math., Mazarredo, 14, 48009 Bilbao, Basque Country, Spain 